\title{
Mechanics of Metals with Phase Changes
}

\section{Los Alamos}


This thesis was accepted by the Department of Chemistry and Biochemistry, Brigham Young University, Provo, Utah, in partial fulfillment of the requirements for the degree of Doctor of Philosophy. The text and illustrations are the independent work of the author and only the front matter has been edited by the IM-1 Writing and Editing Staff to conform with Department of Energy and Los Alamos National Laboratory publication policies.

\section{Copyright () 2000 Jason C. Lashley All Rights Reserved}

An Affirmative Action/Equal Opportunity Employer

This report was prepared as an account of work sponsored by an agency of the United States Government. Neither The Regents of the University of California, the United States Government nor any agency thereof, nor any of their employees, makes any warranty, express or implied, or assumes any legal liability or responsibility for the accuracy, completeness, or usefulness of any information, apparatus, product, or process disclosed, or represents that its use would not infringe privately owned rights. Reference herein to any specific commercial product, process, or service by trade name, trademark, manufacturer, or otherwise, does not necessarily constitute or imply its endorsement, recommendation, or favoring by The Regents of the University of California, the United States Government, or any agency thereof. The views and opinions of authors expressed herein do not necessarily state or reflect those of The Regents of the University of California, the United States Government, or any agency thereof. Los Alamos National Laboratory strongly supports academic freedom and a researcher's right to publish; as an institution, however, the Laboratory does not endorse the viewpoint of a publication or guarantee its technical correctness. 
Mechanics of Metals with Phase Changes

Jason C. Lashley 


\section{Dedication}

This work is dedicated to my wife, Karen, who made a large sacrifice, and to our two children Janessa and Baby Lashley. 


\section{TABLE OF CONTENTS}

DEDICATION

TABLE OF CONTENTS ..................................................................... v

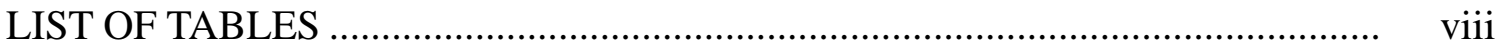

LIST OF FIGURES …......................................................................... ix

ABSTRACT xiii

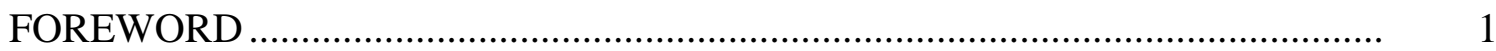

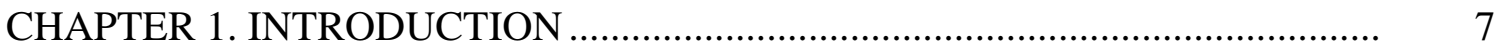

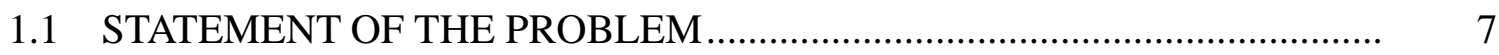

1.2 STRUCTURE AND PROPERTY RELATIONSHIPS ................................ 9

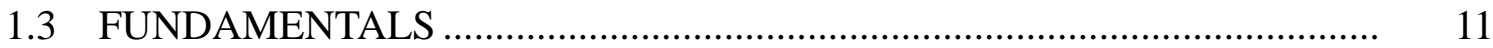

1.3.1 Heat and Entropy ................................................................... 12

1.3.2 Why Are Cryogenic Temperatures Required? ................................. 13

1.4 THE SPECIFIC HEAT OF METALS ..................................................... 14

1.4.1 Phase Changes ............................................................................ 15

1.4.2 First Order Phase Changes ............................................................. 15

1.4.3 Continuous Phase Changes ............................................................. 16

1.4.4 The Martensitic Phase Change ......................................................... 16

1.4.5 Charge Density Waves ........................................................................... 18

1.5 DESCRIPTION OF LOW-TEMPERATURE SPECIFIC

HEAT MEASUREMENTS ................................................................. 19

1.5.1 Semi-Adiabatic Pulse ........................................................................ 19

1.5.2 Isothermal Technique ................................................................... 21

1.5.3 Calorimeter Performance ................................................................ 22

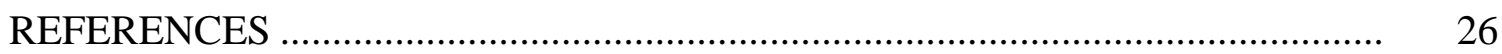

CHAPTER 2. ELASTIC AND THERMODYNAMIC PROPERTIES OF

SINGLE-CRYSTAL AuZn NEAR THE MARTENSITIC TRANSITION .............. 27

2.1 ABSTRACT ......................................................................................... 27

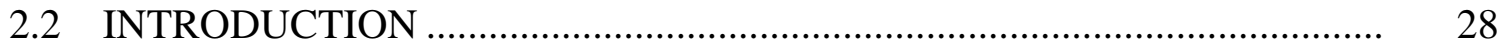

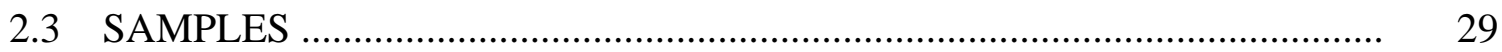

2.4 MEASUREMENTS ....................................................................... 30

2.4.1 Resonant Ultrasound Spectroscopy …........................................ 30

2.4.2 Semi-Adiabatic Calorimetry ........................................................ 31 


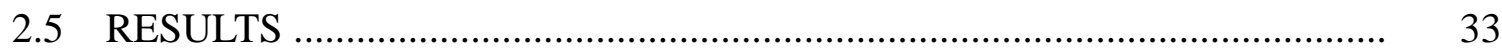

2.5.1 Elasticity .................................................................................... 33

2.5.2 Specific Heat ................................................................................. 37

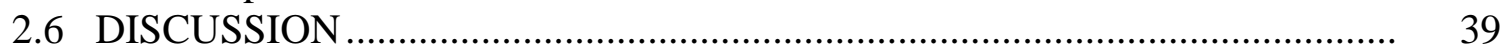

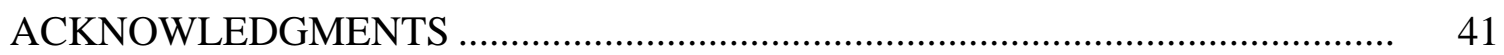

REFERENCES ……............................................................................. 41

CHAPTER 3. THE LOW-TEMPERATURE SPECIFIC HEAT AND

CRITICAL MAGNETIC FIELD OF $\alpha$-URANIUM SINGLE CRYSTALS ............ 43

3.1 ABSTRACT ……................................................................... 43

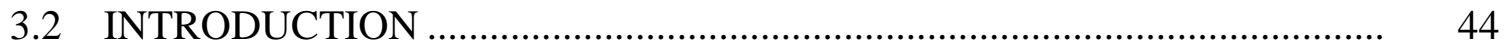

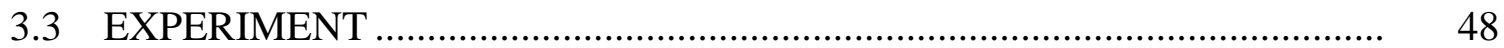

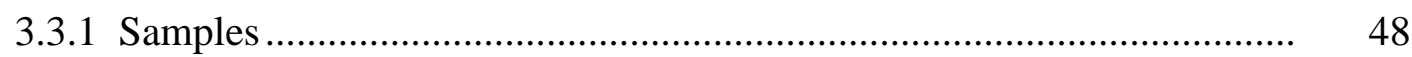

3.3.2 Electrical Resistivity ........................................................................ 49

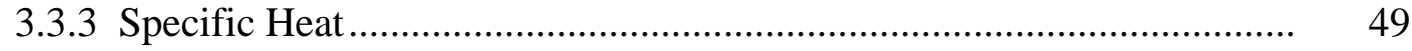

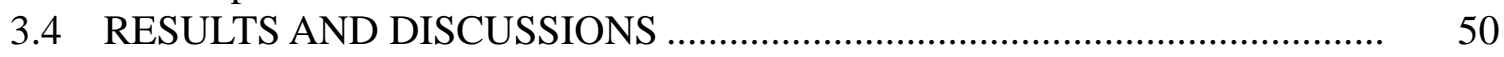

3.4.1 Electricity Resistivity ..................................................................... 50

3.4.2. Specific Heat ………………………………………………....... 53

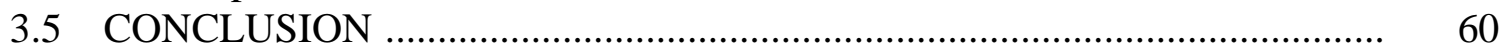

ACKNOWLEDGMENTS …………......................................................... 62

REFERENCES ……......................................................................... 63

CHAPTER 4. A SUCCINCT ACCOUNT OF PLUTONIUM

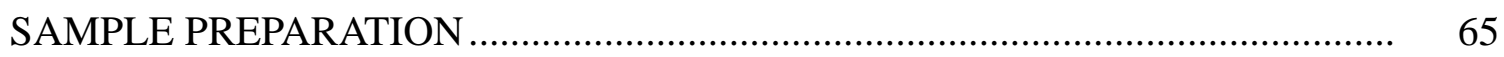

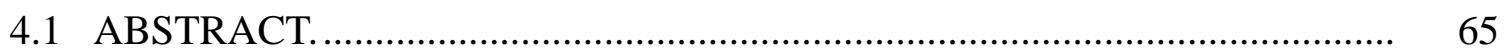

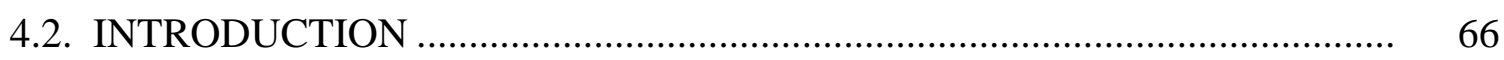

4.3. TECHNIQUES FOR GROWING PLUTONIUM CRYSTALS ......................... 67

4.3.1 Growing Crystals from Molten Plutonium ............................................... 67

4.3.2 Growing Plutonium Crystals in the Solid State ....................................... 69

4.4. LOS ALAMOS PROGRAM FOR GROWING PLUTONIUM CRYSTALS .... 71

4.4.1 Purification .................................................................................. 71

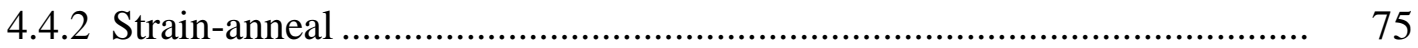

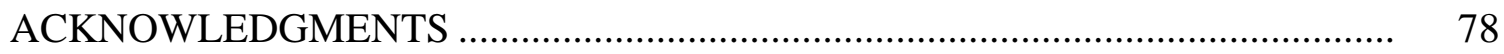

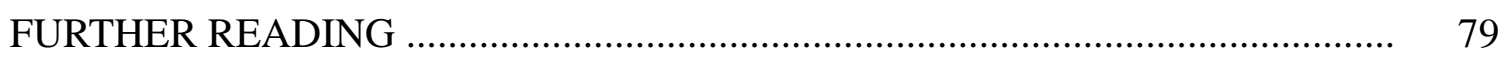

CHAPTER 5. IN-SITU PURIFICATION, ALLOYING, AND

CASTING OF PLUTONIUM METAL ……………...................................... 81

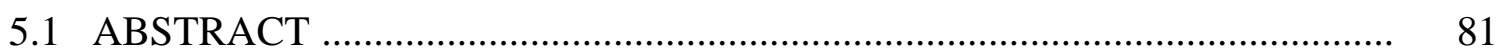

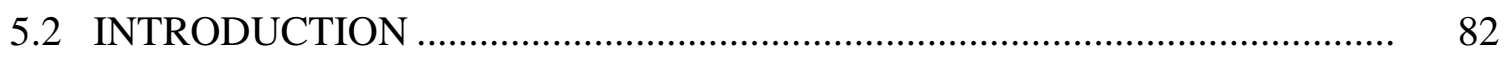

5.3 EXPERIMENTAL PROCEDURE ………………................................. 84

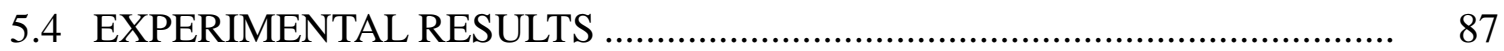

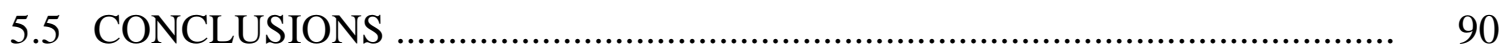

ACKNOWLEDGMENTS …………………………................................ 90

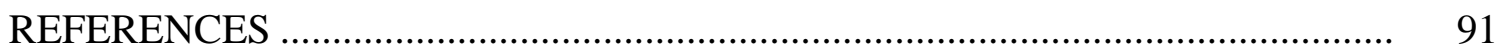


CHAPTER 6. THE CRITICAL RECRYSTALLIZATION STRAIN AND

GRAIN GROWTH OF GALLIUM-STABILIZED $\delta$-PLUTONIUM ..................... 93

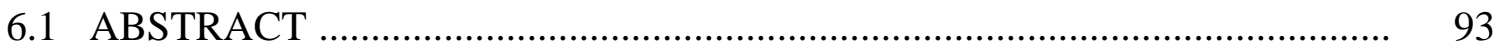

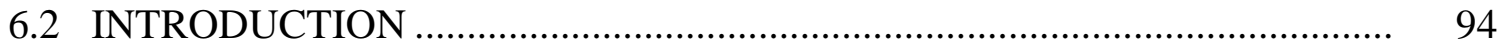

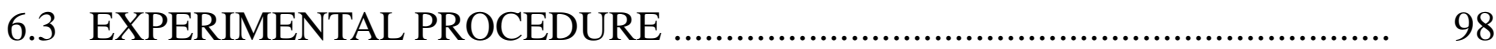

6.3.1 Alloy Preparation ............................................................................. 98

6.3.2 Homogenization and Metallography ................................................... 98

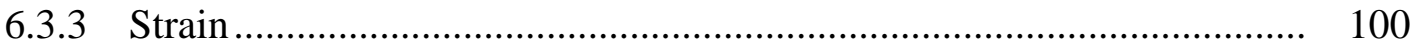

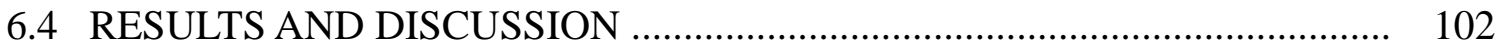

6.4.1 Chemical Purity ...................................................................... 102

6.4.2 Effect of Strain State and Magnitude ................................................ 103

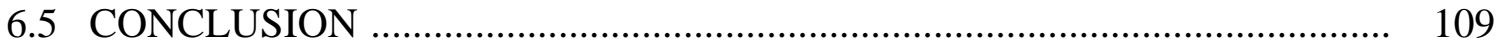

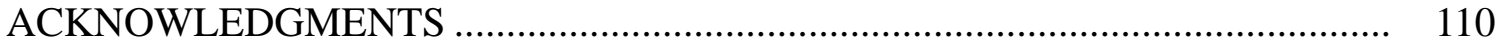

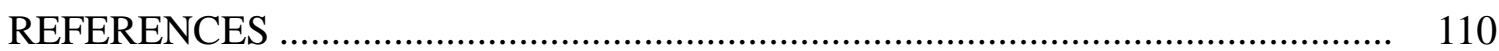

APPENDIX I PURIFIED PLUTONIUM REDUCES BACKGROUND

INTERFERENCE IN NUCLEAR CROSS-SECTION MEASUREMENTS ........... 113

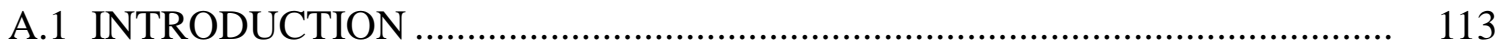

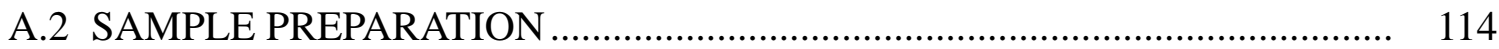

A.3 NUCLEAR CROSS-SECTION MEASUREMENTS .................................... 116

ACKNOWLEDGMENTS ......................................................................... 118

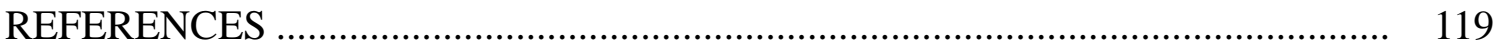




\section{LIST OF TABLES}

Table 2.1. Elastic moduli with estimated errors for single-crystal AuZn .............. 37

Table 3.1 Values of the electronic specific heat ............................................... 46

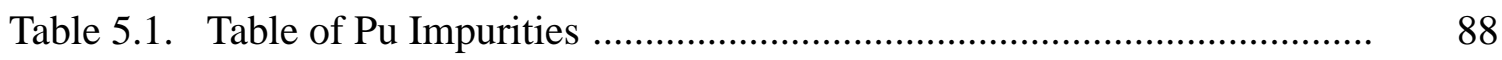

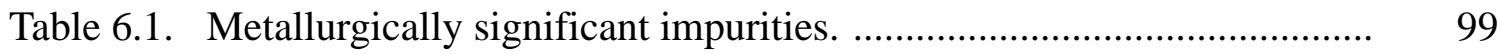

Table 6.2. Critical strains for strain-anneal grain growth................................ 107 


\section{LIST OF FIGURES}

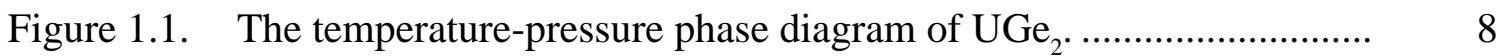

Figure 1.2. Cerium metal. .......................................................................

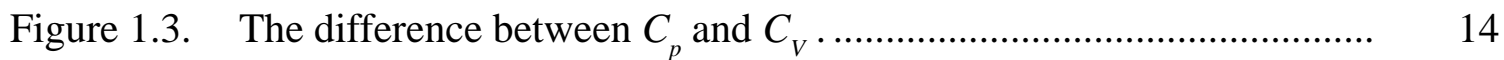

Figure 1.4. First order phase change. ....................................................... 16

Figure 1.5. Continuous phase change. ........................................................ 17

Figure 1.6. Charged density waves. .......................................................... 18

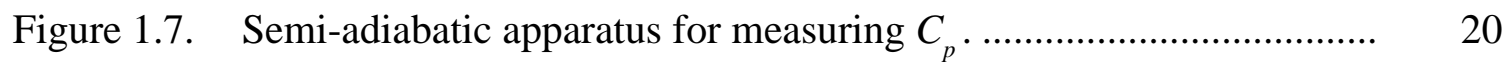

Figure 1.8. The semi-adiabatic pulse technique................................................ 21

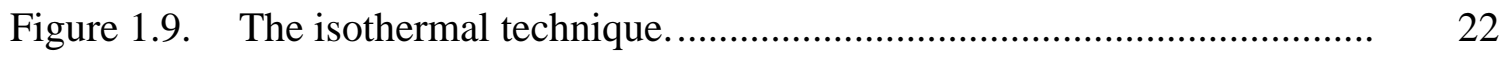

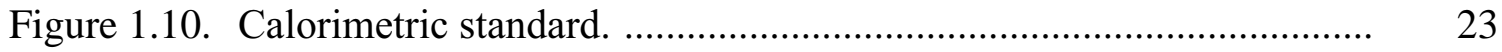

Figure 1.11. Deviation in $C_{p}$ measurements.............................................. 24

Figure 1.12. Copper reference file. .............................................................. 25

Figure 2.1. Resonance frequencies for single-crystal AuZn as a function of temperature.

Figure 2.2. Resonance frequencies for large grain polycrystal AuZn as a function of temperature.

Figure 2.3. Resonance frequencies and typical peak width (internal friction) below $T_{\mathrm{M}}$

Figure 2.4. Shear moduli and anisotropy. ................................................. 36

Figure 2.5. The specific heat of the martensitic transition................................ 38 
Figure 3.1. This figure shows a graphical definition of terms used in describing the resistivity data.

Figure 3.2. Electrical restivity data showing an abrupt resistance drop in the "foot" value at approximately $0.6 \mathrm{~K}$.

Figure 3.3. The $H_{c}(T)$ data evolves into quadratic temperature dependence in the low-temperature limit.

Figure 3.4. The specific heat of single crystal of uranium from $20 \mathrm{~K}$ to $110 \mathrm{~K}$....... 52

Figure 3.5. The low-temperature specific heat of single crystal uranium.

Figure 3.6. The $\alpha_{1}$ and $\alpha_{2} \mathrm{CDW}$ transitions.

Figure 3.7. The $\alpha_{3} \mathrm{CDW}$ transition.

Figure 3.8. The excess heat capacity of the $\alpha_{3} \mathrm{CDW}$ transition.

Figure 3.9. The electronic heat capacity and Debye temperature.

Figure 3.10. The low-temperature specific heat with the hyperfine contribution subtracted.

Figure 4.1. Polycrystalline plutonium.

Figure 4.2. The low-gallium composition of the plutonium-gallium binary phase diagram.

Figure 4.3. Levitation zone-refining furnace.

Figure 4.4. Vacuum distillation of plutonium metal.

Figure 4.5. Biaxial distress in a plutonium disk.

Figure 5.1. In situ americium distillation, alloying, and chill-casting step.

Figure 5.2. Chill-Cast $\mathrm{Pu}$ Microstructure.

Figure 6.1. The low gallium composition of the plutonium-gallium binary phase diagram.

Figure 6.2. Punch-stretching experiment.

Figure 6.3. Aluminum disk after stretching to a dome height of $11.0 \mathrm{~mm}$. 
Figure 6.4. Strain calibration from an aluminum disk.

Figure 6.5. The calculated effective strains for aluminum disks. ....................... 105

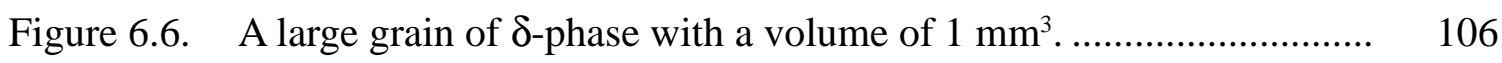

Figure A.1. Sample preparation. ................................................................... 115

Figure A.2. The plutonium sample background (i.e., no incident neutron beam)

Figure A.3. A background (i.e., no incident neutron beam), low-energy photon spectrum. 


\title{
MECHANICS OF METALS WITH PHASE CHANGES
}

\author{
Jason C. Lashley
}

\begin{abstract}
New experimental data is presented on some exotic metals that exhibit phase changes at cryogenic temperatures. The types of phase changes that were detected in the specific heat data range from martensitic (diffusionless) transitions to superconducting transitions. In addition, the charge density wave (CDW) state in uranium metal was detected in the specific heat. Specific-heat measurements were made in zero-magnetic field using an apparatus capable of obtaining temperatures as low as $0.4 \mathrm{~K}$. Calibration performed on this apparatus, using a single-crystal copper sample, show its accuracy to be 0.50 per cent, while the resolution was better than 0.1 per cent. Our measurements demonstrate that similar high precision and accurate specific-heat measurements can be obtained on milligram-scale samples. In Chapters 2 and 3, specific-heat measurements are presented for the $\mathrm{B} 2(\mathrm{CsCl}$ structure) alloy $\mathrm{AuZn}$ and for $\alpha$-uranium (orthorhombic symmetry). The AuZn alloy exhibits a continuous transition at $64.75 \mathrm{~K}$ and an entropy of transition of $\left(\Delta S_{\mathrm{tr}}\right) 2.02 \mathrm{~J} \mathrm{~K}^{-1} \mathrm{~mol}^{-1}$. Calculation of the Debye temperature, by extrapolating of the high temperature phase elastic constants to $\mathrm{T}=0 \mathrm{~K}$ yields a value of $207 \mathrm{~K}( \pm 2 \mathrm{~K})$, in favorable agreement with the calorimetric value of $219 \mathrm{~K}( \pm 0.50 \mathrm{~K})$, despite the intervening
\end{abstract}


martensitic transition. Reported results for single-crystal $\alpha$-U show a low-temperature limiting $\Theta_{D}$ of $256 \mathrm{~K}( \pm 0.50 \mathrm{~K})$ and four low-temperature anamolies: a superconducting transition below $1 \mathrm{~K}$, an electronic transition at $22 \mathrm{~K}$, and two anamolies at $38 \mathrm{~K}$ and at $42 \mathrm{~K}$ indicative of the CDW state. In order to continue the study of the actinide series of elements, a program was initiated to first purify and then grow single crystals of plutonium. Accordingly, the focus of Chapters 4 through 6 will be a description of plutonium sample preparation. In this program plutonium metal was purified via zone refining, using a levitated molten zone to minimize the introduction of impurities. Several impurities were reduced to levels below that of instrument detection limits. Results indicate that six sequential zone refining passes are required to obtain metal with $130 \mathrm{ppm}$ total impurities (excluding $\mathrm{N}, \mathrm{O}, \mathrm{F}, \mathrm{Cl}$, and $\mathrm{Br}$ ). Small single crystals with a volume of $1 \mathrm{~mm}^{3}$ were grown of $\delta$-plutonium by a strain-anneal technique. The values obtained for the critical strain in the strain-anneal experiments are in favorable agreement with values obtained by other researches using loading methods other than biaxial loading. 
FOREWORD

For out of olde feldes, as men seith, Cometh al this newe corn fro yeer to yere; And out of olde bokes, in good feith, Cometh al this newe science that men lere.

- Chaucer

From a particle physics viewpoint, solid-state chemistry and physics is the science of dirt. Whereas the particle physicist looks at collisions between individual particles, the solid-state scientist has $10^{23}$ particles to contend with. Avogadro's number of particles makes dirt. Because no two full shovels of dirt are the same, how can one expect to obtain clean experimental results? One example that helped answer this question was the discovery of the Josephson effect in 1962 [1]. This effect occurs by applying a direct current electric potential across a junction between two superconducting solids. A voltage drop across the junction causes an oscillating alternating current with angular frequency, $\omega$, related to Planck's constant by Equation (0.1)

$$
\omega_{J}=\frac{2 e V}{\hbar},
$$


where $e$ is the electron charge and $V$ is the voltage. This result forms a standard for making highly accurate voltage measurements and precise measurements of Planck's constant from solid superconducting samples [2]. This effect occurs for any two superconducting solids regardless of the purity. Many other physical properties of solids are extremely sensitive to small changes in external parameters such as pressure and doping, and perhaps the large number of empirical physical constants in the branch of solid-state science forms a testament to the fact that properties often change from sample to sample. Consequently, there is always a need for high quality samples used in investigating new phenomena or to use in revisiting old phenomena with better samples. For these reasons, I have chosen to do a dissertation that addresses both the measurement and sample preparation aspects. In this context the boundaries between the individual branches of physical science become artificial, and a framework of a close collaboration between chemists, physicists, and metallurgists is required to solve problems. This is the philosophy adopted for the present work, in which I investigate two important classes of related materials: shape memory alloys and the actinide metals.

A priori, it may seem that there is little in common between shape memory alloys and the actinides. However, in the study of both the shape memory alloys and the actinide series of elements one cannot avoid phase transformations, and in particular the martensitic $^{1}$ transformation [3]. Some of the current problems associated with these materials are the development of theoretical models that describe both the electronic properties and the evolution of complex microstructures. Such problems are worthy of study because the

\footnotetext{
${ }^{1}$ Martensite is after the German scientist Martens. A martensitic transformation occurs as atoms move less than their interatomic distance, and retains a relationship with their neighbors during the phase change. It is often referred to as a diffusionless or displacive transition.
} 
shape memory alloys form an important class of technological materials. For example, shape memory alloys are widely used as a material for eyeglass frames and for artificial hip joints. Thus, a comprehensive knowledge of the thermodynamics of martensitic transitions helps to form a theoretical foundation of this important class of materials.

The actinide series of elements and beyond are the last unexplored area of the Periodic Table. This series is characterized by the presence of $5 f$-electrons, displaying narrow band behavior at the Fermi energy. A description of the electronic structure is the current testing ground of modern solid-state theory. A comprehensive knowledge of the actinide elements contributes to a "first principles" understanding of both nuclear and solid-state problems. Much of this information, such as nuclear cross-section data, has been previously obtained from the nuclear testing era. Therefore, in principle one can argue that a detailed knowledge, obtained from experimentation, can contribute to the reduction of the global nuclear danger by reducing the need for nuclear testing.

Keeping the preceding paragraphs in mind, I now summarize the specific problems addressed in the current work.

Problem 1: The shape memory effect is associated with alloys that exhibit a martensitic transformation. A phenomenological theory of the martensitc transformation has evolved from a compilation of crystallographic data and has done good service in disseminating clear notions in this science, however the thermodynamic properties of the martensitic transition are only known imprecisely for many martensites. For example, there are many examples in the literature of thermodynamic properties measured by differential scanning calorimetry (DSC). While DSC is well suited for the detection of phase changes, it is often over extended (based upon the resolution and accuracy limits) 
in describing the order of a martensitic transition. Because there are few examples where high quality thermodynamic data (resolution better than 0.1 per cent and accuracy better than 0.5 per cent) have been accumulated on these materials, it is useful to measure the specific heat of the martensitic transformation by semi-adiabatic calorimetry with due regard for the configurational entropy. The entropic contributions associated with the martensitic transformation describe the atomic displacements in a transition. When this information is combined with other measurements such as the elastic moduli, it forms a foundation for theoretical models.

Problem 2: There are other types of phase changes that are related to the martensitic transformation. The charge density wave (CDW) is a broken symmetry state in which the crystal interacts with an underlying potential, thereby lowering its overall energy. This phenomenon is often associated with a lattice distortion. Today an understanding of the density waves in solids follows the pioneering work of Peierls [4], Frölich [5] and Overhauser [6]. Although CDW have been reported in sodium and potassium, uranium is the only element to display both a CDW state and a superconducting transition [7-10]. The low-temperature specific heat has been investigated many times on polycrystalline uranium and on imperfect single crystals. In these investigations, there has been a 16 per cent discrepancy between the Debye temperatures obtained from elastic moduli and calorimetry. New single crystals with unexpected mechanical properties became available to us. Because these single crystals were prepared by electrochemical transport in the $\alpha$-phase, we anticipated that they would be relatively strain free and that the characteristics of the CDW state might be more prominent in these crystalss. Therefore, we revisit 
the low-temperature specific heat to obtain a Debye temperature, report the entropy for each of the CDW transitions, and search for the superconducting transition.

Problem 3: Currently, there is a need for single crystals of plutonium metal in order to investigate the unusual properties of this important element. To date, all of plutonium's properties except for the elastic anisotropy have been deduced from polycrystalline samples [11]. Because each of these properties exhibits a high sensitivity to the presence of trace elemental impurities and alloy composition, samples must be prepared from metal of the highest chemical purity. Therefore, we present new purification techniques that have produced high purity material, and describe some neutron cross-section measurements made on this material. We then present the current status of efforts to obtain single crystals of plutonium by solid-state strain-anneal techniques.

\section{REFERENCES}

[1] B. D. Josephson, Phys. Lett. 1, 251 (1962).

[2] W. H. Parker, Phys. Rev. 177, 639 (1969).

[3] S. S. Hecker, “Introductory Remarks" Martensite Workshop, Los Alamos, New Mexico, July 2000.

[4] R. Peierls, Ann. Phys., 4, 121, (1930).

[5] H. Frölich, Proc. R. Soc. London A, 223, 296, (1954).

[6] A. W. Overhauser, Phys. Rev. B., 3, 3173, (1971).

[7] A. W. Overhauser, Adv. Phys., 27, 343, (1978).

[8] H. Yong Gyoo and A. W. Overhauser, Phys. Rev. B., 39, 3037, (1989). 
[9] L. Fast, O. Eriksson, B. Johansson, J. M. Wills, G. Straub, H. Roeder, and L. Nordström, Phys. Rev. Lett., 81, 2978, (1998).

[10] G. H. Lander, E. S. Fisher, and S. D. Bader, Adv. Phys., 43, 1 (1994).

[11] H. M. Ledbetter and R. L. Moment, Acta. Met., 24, 891 (1976). 


\title{
1 \\ INTRODUCTION
}

Great ideas initiate as heresy and terminate as mythology.

- N. Bohr

\author{
1.1 STATEMENT OF THE PROBLEM \\ 1.2 STRUCTURE AND PROPERTY RELATIONSHIPS \\ 1.3 FUNDAMENTALS \\ 1.3.1 Heat and Entropy \\ 1.3.2 Why are Cryogenic Temperatures Required? \\ 1.4 THE SPECIFIC HEAT OF METALS \\ 1.4.1 Phase Changes \\ 1.4.2 First Order Phase Changes \\ 1.4.3 Continuous Phase Changes \\ 1.4.4 The Martensitic Phase Change \\ 1.4.5 Charge Density Waves \\ 1.5 DESCRIPTION OF LOW-TEMPERATURE SPECIFIC HEAT MEASUREMENTS \\ 1.5.1 Semi-Adiabatic Pulse \\ 1.5.2 Isothermal Technique \\ 1.5.3 Calorimeter Performance \\ REFERENCES
}

\subsection{STATEMENT OF THE PROBLEM}

In the low-temperature limit, sudden changes in the ground state wavefunction of a system resulting from small perturbations are called quantum phase transitions [1,2].

Similar to ordinary critical phenomena, the behavior of a material near a quantum phase 
transition can be quite anomalous - a situation referred to as quantum criticality. Anomalous behavior near a quantum critical point has recently been demonstrated for the heavy fermion $\mathrm{UGe}_{2}[3]$, where a superconducting transition has been detected in a ferromagnetic state, shown in Figure 1.1. Although the observed anomalous behavior is not yet completely understood, it has been attributed to the unique electronic structure of these materials.

After fifty years of multidisciplinary research one might think that we could simply catalog the engineering properties of metals and other materials and use this to know, for example, how their properties change with time. However their properties depend too sensitively on things such as impurities, temperature, and method of fabrication to be predictable. Thus, we must rely on experimentation to answer such complex problems

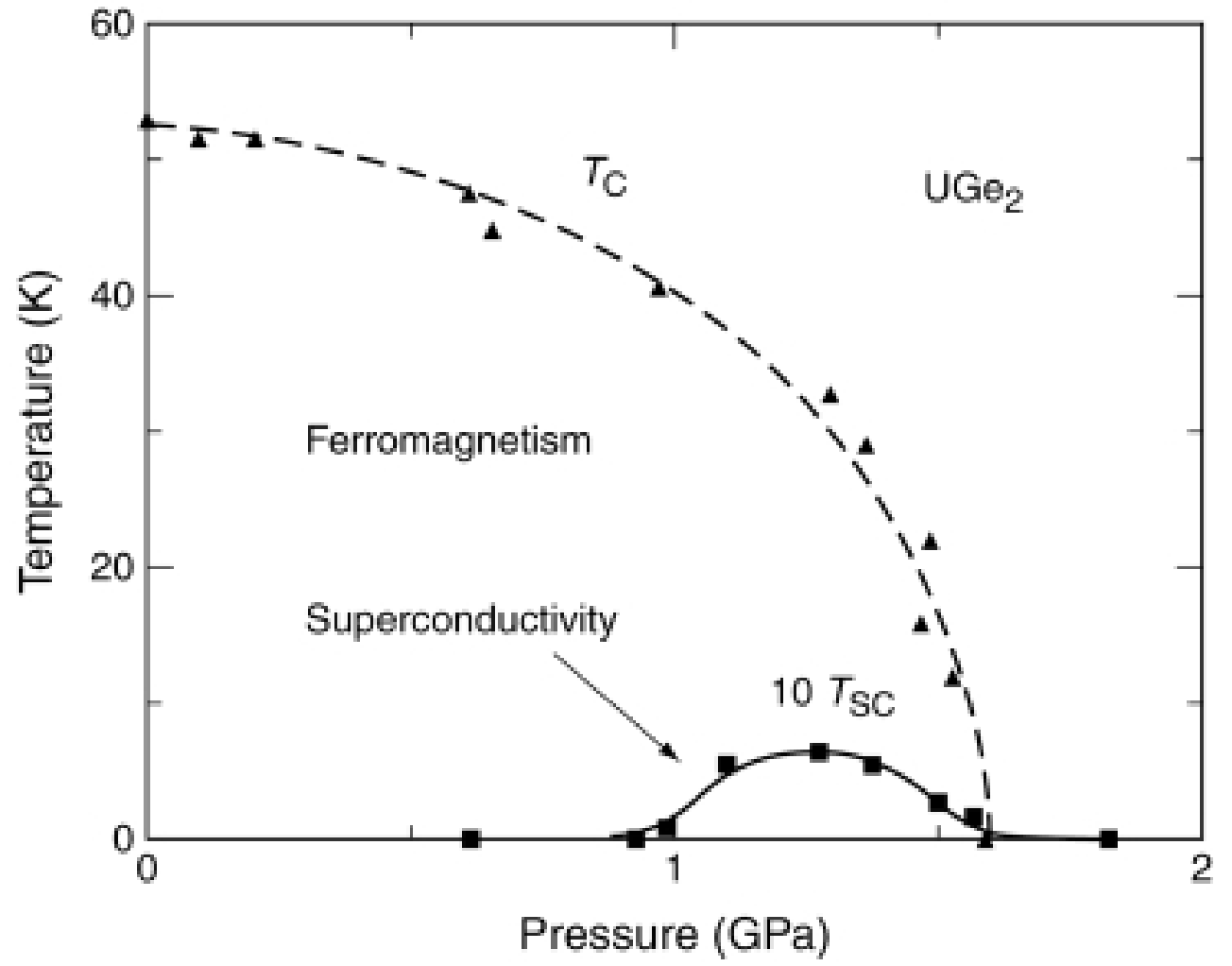

Figure 1.1. The temperature-pressure phase diagram of $\mathrm{UGe}_{2}$. [Reprinted by permission from Nature 406587 (2000)]. 
whose solutions are deeply rooted in the intricate connection between the structure and properties of metals.

\subsection{STRUCTURE AND PROPERTY RELATIONSHIPS}

Metal properties include the vibrations of atoms and groups of atoms, the distribution of electron density, and a Fermi surface arising from the Pauli exclusion principle operating on conduction electrons. In metals, groups of atoms arranged in a repetitive-symmetric array form a grain (crystal), the smallest unit of a metal to retain the fundamental properties of the bulk solid. A grain extends throughout space until it intersects another group of atoms that has a different crystallographic orientation, shown in Figure 1.2. The intersection of two or more grains is called a grain boundary, and the bulk metal is composed of numerous grain boundaries that are formed by many crystals (polycrystalline) the natural state of most elemental metals. Because a single crystal is the smallest unit of a metal to retain the fundamental properties of the metal, experiments using single crystals convey the most fundamental information. In contrast, measurements on polycrystalline samples often do not reveal anisotropic properties because probing a polycrystalline sample is subject to averaging the data over all crystallographic directions. For these reasons, all of the samples that were measured in this work were high quality single crystals.

Matter is composed of both electrons and nucleons. The electronic structure of metals governs properties such as elasticity, magnetism, bonding, and electrical conductivity [4-6]. Generally, vibrational properties dominate the thermal properties of a solid such as thermal expansion and specific heat, although there are significant electronic 


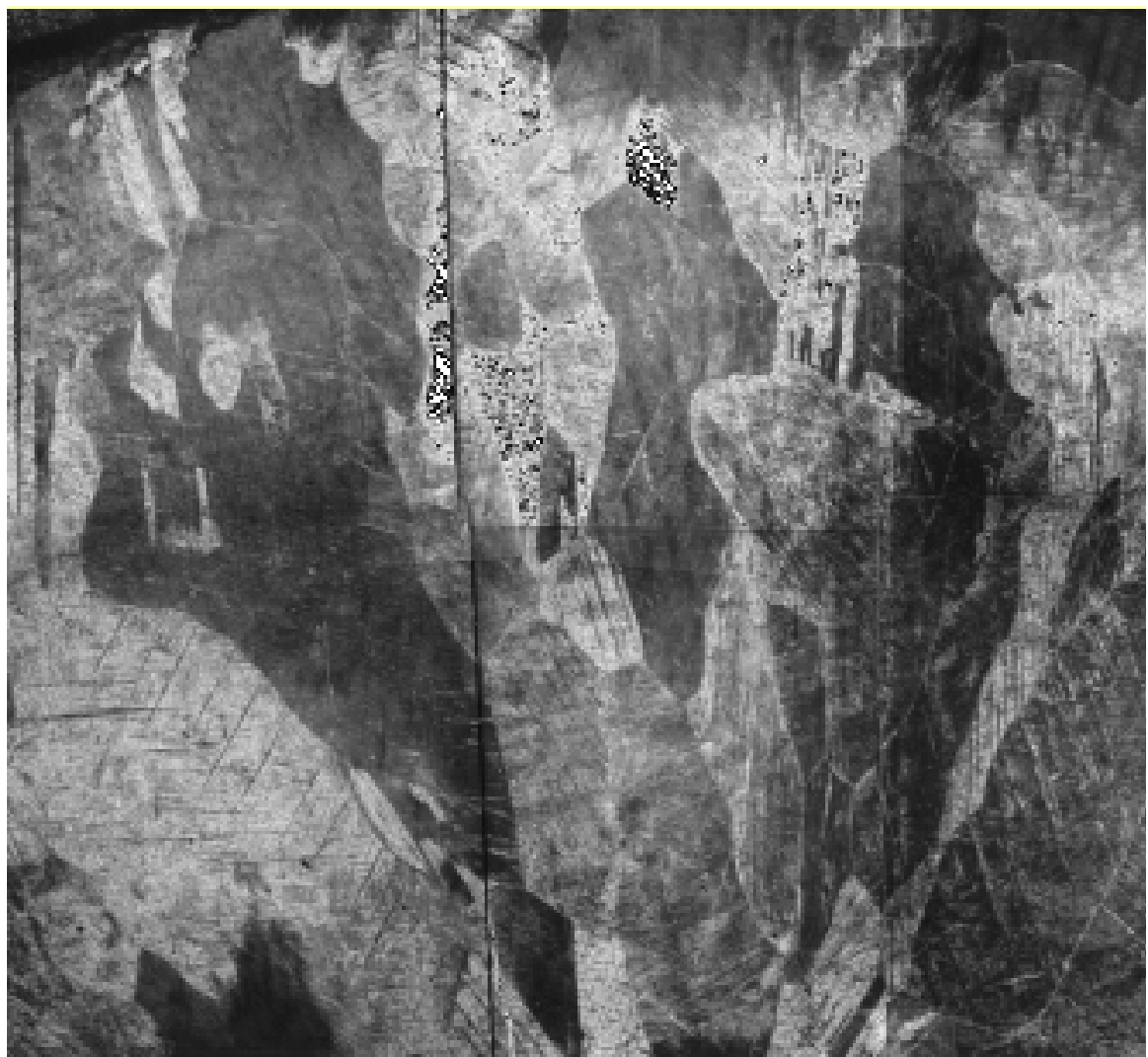

Figure 1.2. Cerium metal.

contributions to the specific heat at low temperatures and at phase changes. Therefore specific-heat measurements will be the focus in Chapters 2 and 3. Because specific-heat measurements on phase changes in metals involve the detection of energy, it is useful to note that there are various forms of stored energy ${ }^{1}$ in metals [7]. Energy is often stored in the lattice as defects, and it is commonly found in samples that become cold-worked ${ }^{2}$ during the preparation stages. The stored energy in a sample is usually released in the form of heat at a given temperature. The presence of stored energy is undesirable when making an absolute measurement. Thus, sample preparation methods that minimize these

${ }^{1}$ The term energy was first introduced by Thomas Young in 1805, but the word "force" was commonly used until the mid-1800s.

${ }^{2}$ The cold-worked state is any damaged condition of a metal, regardless of the process that produced the condition. 
effects are necessary to produce samples that are suitable to meet the stringent demands of solid-state measurements. To better understand these sample requirements, it is necessary to begin with a discussion of the fundamental thermodynamic relationships of phase changes. The preparation of samples will be the focus of chapters 4 through 6 .

\subsection{FUNDAMENTALS}

A failure in the thermodynamic stability of a system resulting in a transformation of a macroscopic ensemble of thermodynamic objects into another distinct ensemble defines a phase change. In order for a phase change to proceed spontaneously at a given temperature and pressure, it must be associated with a decrease in free energy. There are a few ways to determine the free energy, depending on the conditions of the thermodynamic system under consideration. For example, in familiar phase changes such as melting and freezing, two or more phases can coexist at a given temperature and pressure. For this reason a measure of the Gibbs free energy change, $\Delta G$, which relates the free energy of a system at constant temperature and pressure, is applicable to many situations

$$
\Delta G=\Delta H-T \Delta S
$$

where $\Delta H$ is the enthalpy change, and $\Delta S$ is the entropy change. The chemical potential is a partial molar quantity that is used as a measure of molar free energy, and it is given by, $\mu=\left(\frac{\partial G}{\partial n}\right)_{p, T}$, where $\mu$ is the chemical potential for a pure substance, $T$ is the thermodynamic temperature, and $n$ is the number of moles. Thus, for a pure substance the chemical potential is a measure of its molar Gibbs free energy [8]. However, there are cases when it is necessary to express the equation of state for a solid by using a form of the chemical potential at constant volume and temperature conditions. Under these circumstances, the 
chemical potential can be written in terms of the Helmholtz function, $A$, which is also a measure of free energy where the chemical potential becomes $\mu=\left(\frac{\partial A}{\partial n}\right)_{V, T}$.

\subsubsection{Heat and Entropy}

Heat, $q$, fails to be a state function ${ }^{3}$ because it is energy in transit. The flow of heat in or out of a system, causes a change in temperature, $d T$, and the specific heat is calculated from

$$
C=\frac{\delta q}{d T}
$$

For an isochoric (constant volume) process, $C=C_{V}$, and the specific heat is based on the thermodynamic relationship, $C_{V}=\left(\frac{\partial U}{\partial T}\right)_{V}$, and for an isobaric (constant pressure) process $C=C_{p}$, the specific heat is $C_{p}=\left(\frac{\partial H}{\partial T}\right)_{p}$, where $H$ is the enthalpy. The thermodynamic relationship between entropy and the specific heat is obtained through the Maxwell relation, $\left(\frac{\partial S}{\partial T}\right)_{p}=\frac{C_{p}}{T}$, and the entropy change for the isobaric temperature change is obtained by separation of variables and integrating the specific heat between two temperatures, $T_{1}$ and $T_{2}$

$$
\Delta S=\int_{T_{1}}^{T_{2}} \frac{C_{p}}{T} d T
$$

\footnotetext{
${ }^{3}$ In order for a function, $f(x)$, to be a state function, the differential $d f$ must be an exact differential. Thus, one of the following statements must be satisfied in order for a function to be a state function:

$d f$ is an exact differential, $\oint d f=0$, or $\int_{a}^{b} d f=f(b)-f(a)$, path independent.
} 
The entropy change for the reversible equilibrium phase change (first-order) is calculated from

$$
\Delta S_{t r}=\frac{\Delta H_{t r}}{T_{t r}}
$$

where $\Delta S_{t r}$ is the entropy change of the transition, $\Delta H_{t r}$ is the enthalpy change for the phase change, and $T_{t r}$ is the transition temperature. For a non first-order phase change, the entropy change is calculated as in equation (1.3) using the excess specific heat above the background lattice contribution. The collective entropic contributions are calculated from

$$
\Delta S=\sum_{i} \frac{\Delta H_{i}}{T_{t r}}+\int \frac{C_{p}(T)}{T} d T
$$

where the sum is over any first-order equilibrium phase changes.

\subsubsection{Why are cryogenic temperatures required?}

The specific heat at constant volume and pressure are not equal. The primary importance is that $C_{p}$ is typically measured, while theory is derived for constant volume conditions. They are approximately at low-temperatures, and at higher temperatures the correction is based on the thermodynamic relationship

$$
C_{p}=C_{V}+\frac{\alpha^{2} V T}{\kappa}
$$

where $\alpha$ is the isobaric coefficient of thermal expansion defined by the relationship, $\alpha=\frac{1}{V}\left(\frac{\partial V}{\partial T}\right)_{p}$, and $\kappa$ is the coefficient of compressibility defined as $\kappa=-\frac{1}{V}\left(\frac{\partial V}{\partial P}\right)_{T}$. The point of deviation between $C_{p}$ and $C_{V}$ depends on the material. For example, the difference between $C_{p}$ and $C_{V}$ for the metals $\mathrm{Na}$ and $\mathrm{Al}$ is shown in Figure 1.3 where the experimental curves of $C_{p}$ and $C_{V}$ were calculated from equation (1.6). It is interesting to 
note that the difference in $C_{p}$ and $C_{V}$ becomes noticeable at approximately $100 \mathrm{~K}$. For these reasons, low-temperature fits of specific-heat data provide useful parameters that are directly comparable to theory because in most cases the ground state of a system is reached.

\subsection{THE SPECIFIC HEAT OF METALS}

The specific heat of metals at low temperatures is often written as the sum of lattice (phonon) and electronic contributions [9-11]:

$$
C=\gamma T+B_{3} T^{3}
$$

where the electron specific heat $(\gamma)$ and $B_{3}$ are constants that are characteristic of a material. In practice the electronic specific heat is obtained from a low-temperature fit to Equation (1.8). At low temperatures $\left(\Theta_{D} / 50\right.$ as a general rule) the electronic term domi-

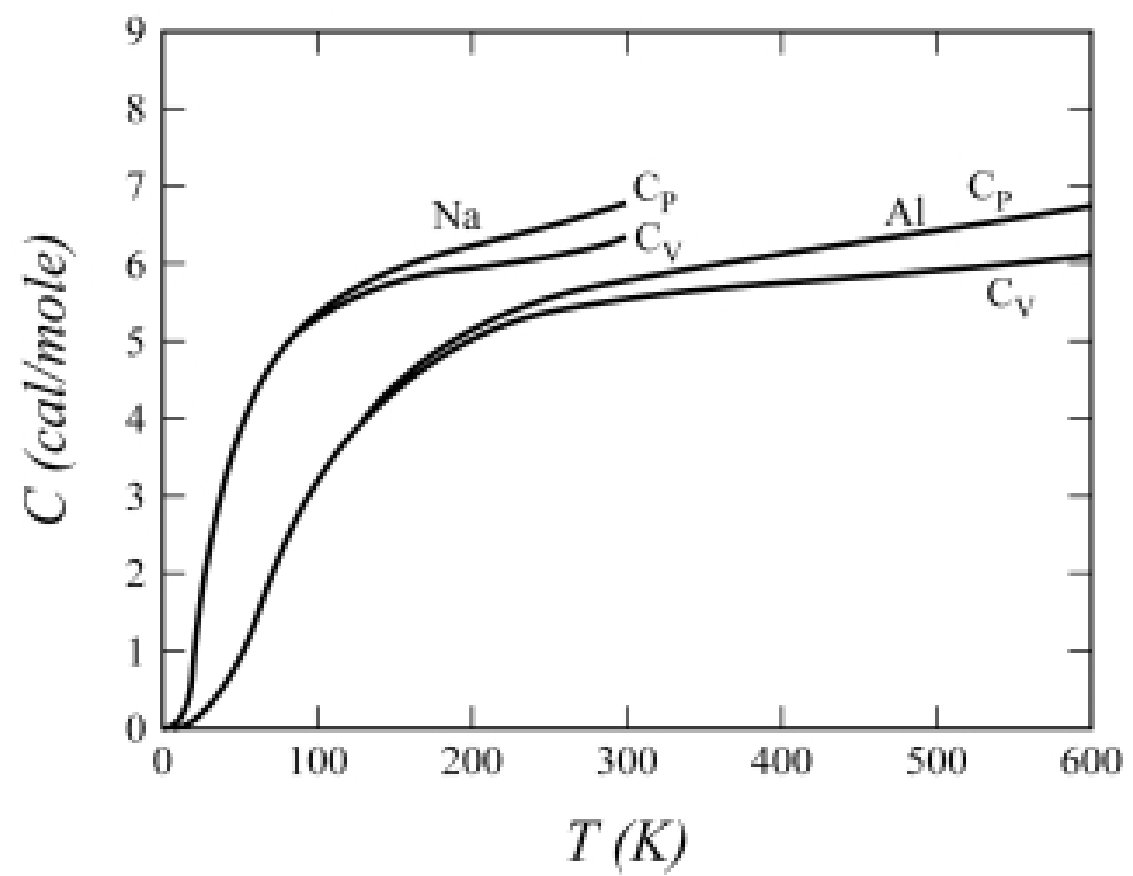

Figure 1.3 The difference between $C_{p}$ and $C_{V^{*}}$. Reprinted with permission, “Thermodynamics of Crystals," Dover Publications, Inc.] 
nates, and it is customary to exhibit experimental values of $C$ as a plot of $C / T$ versus $T^{2}$ because $\gamma$ and $B_{3}$ are seen as the intercept and slope, respectively

$$
C / T=\gamma+B_{3} T^{2}
$$

\subsubsection{Phase Changes}

The mathematical picture of the behavior at the transition temperature define the order of a phase change. There are basically two classifications of phase changes: first order and continuous. In a first-order phase change, both the entropy and the volume, which are first derivatives of the Gibbs function, are discontinuous at the transition temperature. Conversely, there are phase changes in which the volume, entropy, and Gibbs function remain invariant at the transition temperature. In this case the second derivatives of the Gibbs function, $C_{p}, \alpha$, and $\kappa$ exhibit a power law singularity (but are not discontinuous). This result defines a continuous phase change, and each type of phase change differs in shape arising from either discontinuities or singularities in either the first or second derivatives of the chemical potential.

\subsubsection{First Order Phase Changes}

The behavior of $C_{p}$ for a first-order phase change is shown in Figure 1.4. Thus, $C_{p}$ tends to infinity at the transition temperature where the two phases coexist. A few common features that are attributed to first-order transitions are abrupt changes of entropy and volume, and the first derivatives of the Gibbs function are discontinuous at the transition temperature. Any phase change satisfying these criteria is labeled as first order. 


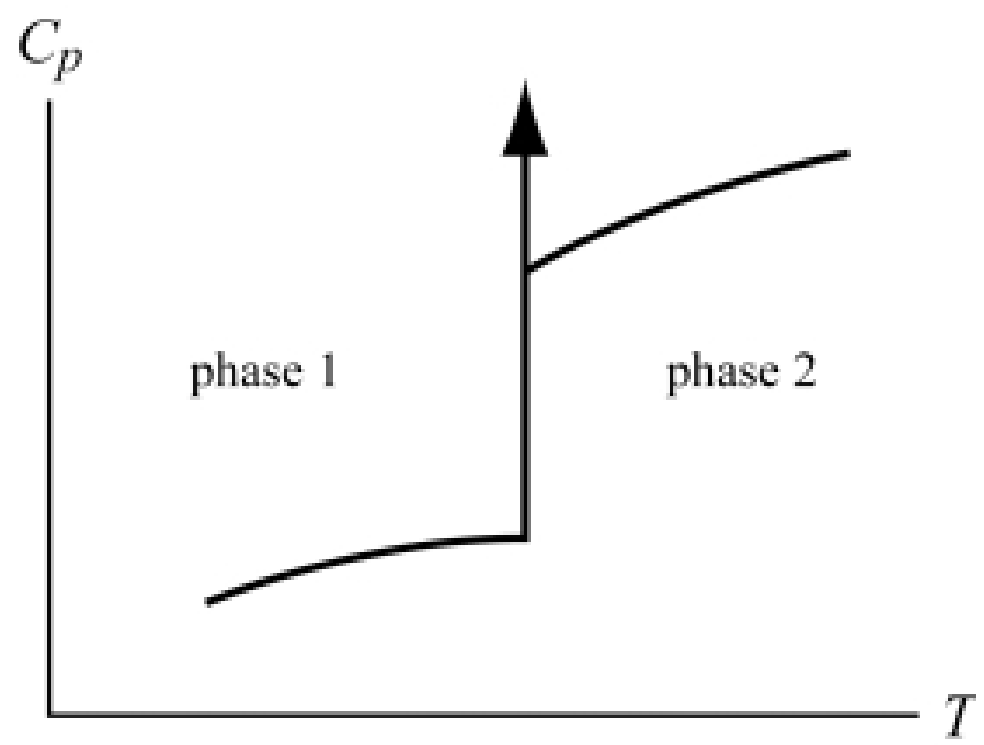

Figure 1.4. First order phase change.

\subsubsection{Continuous Phase Changes}

Renormalization group models have shown that for phase changes greater than order one, the historical Ehrenfest relations fail to apply. The quantities thought to be discontinuous are in fact power law singularities given by some empirically determined exponent $\left|T-T_{c}\right|^{-\beta}$. Consequently, a second class of phase changes called second order transitions are correctly called continuous transitions because there are no discontinuities. A graphical example of this type of phase change in $C_{p}$ is shown in Figure 1.5. Continuous phase changes are always associated with critical phenomena. Because the thermodynamics of the diffusionless phase change comprise a large portion of this work, we will close with a brief description of the martensitic transition and the charge density wave.

\subsubsection{The Martensitic Phase Change}

Phase changes where there are no long-range movements of atoms define the martensitic phase transformation. For most cases, atoms move less than an interatomic distance and retain the relationship with their neighbors during a phase transformation. This 


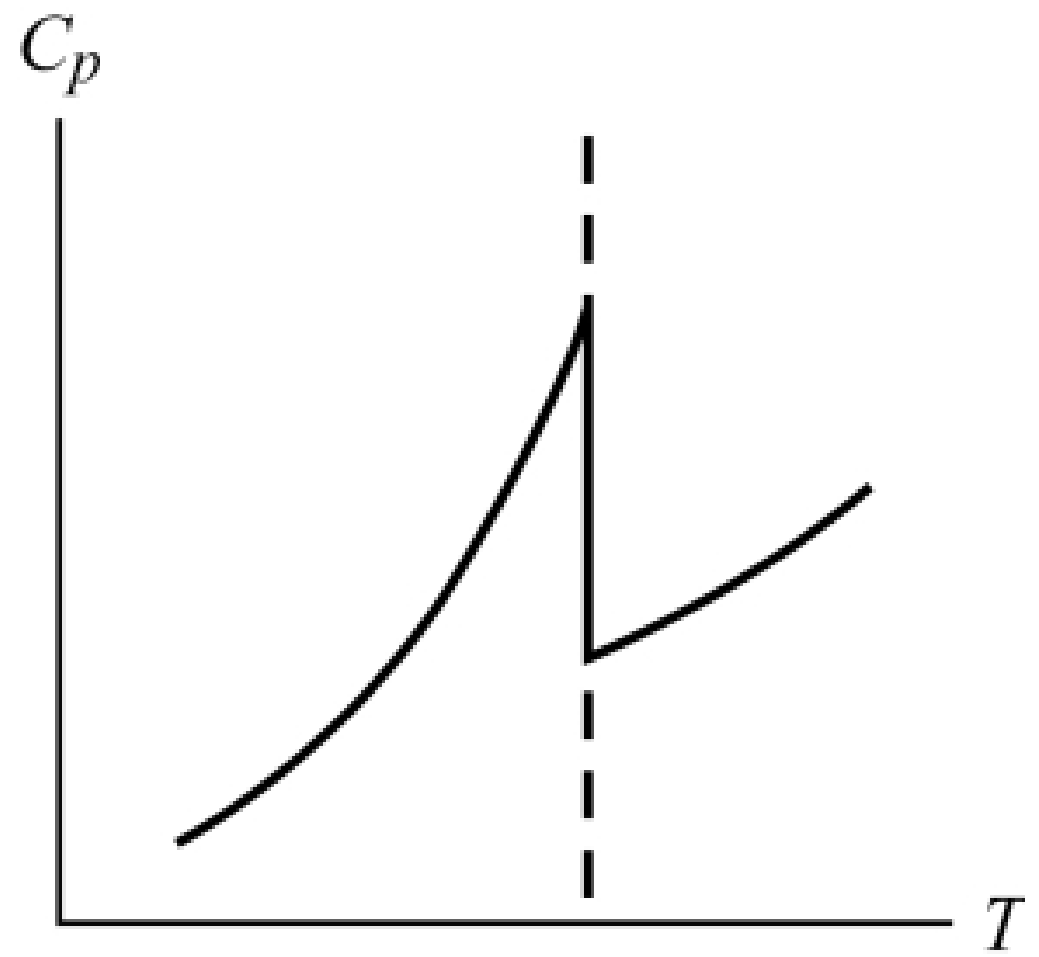

Figure 1.5. Continuous phase change. Critical phenomena, such as quantum phase transitions and nuclear ordering, are always associated with this type of phase change.

property gives rise to special group theoretical connections between two phases. In many cases, martensitic phase transformations occur at temperatures below $100 \mathrm{~K}$, and in other cases a martensite is produced at higher-temperatures (e.g., welding 304 stainless steel). Martensitic transformations are usually distinguished based on their crystallographic characteristics. In the absence of crystallographic data, it is nearly impossible to characterize a martensitic transformation from specific heat data because one cannot characterize such a transition based on the order. Despite the fact that most martensitic transformations have been described in the literature as first order, one cannot characterize this transformation in terms of order in a unique manner. 


\subsubsection{Charge Density Waves}

The charge density wave is another type of diffusionless phase change that often involves a lattice distortion $[11,12]$. Charge density waves typically occur in low-dimensional crystal systems, and the driving force is that the total energy of the system is lowered. Although the CDW shows up clearly in the specific heat data, one cannot attribute these anomalies to the CDW in the absence of neutron or x-ray diffraction data. The CDW has been observed in quasi one-dimensional systems and transition metal dichalcogenides. A schematic drawing showing the CDW is shown in Figure 1.6. In the one dimensional model, a commensurate structure (a) is defined by the atomic positions being related to the underlying potential by a rational number, and in the incommensurate structure (b) the two are related by an irrational number. The change from one structure to another shows up in the specific heat data and it gives rise to disorder in the crystal. From a structural property standpoint, this disorder is connected to changes in the physical properties such as lattice parameter, elastic properties, and thermal expansion.

(a)

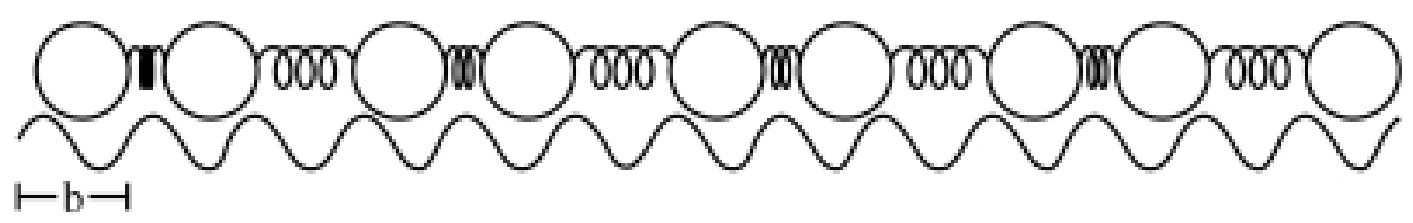

(b)

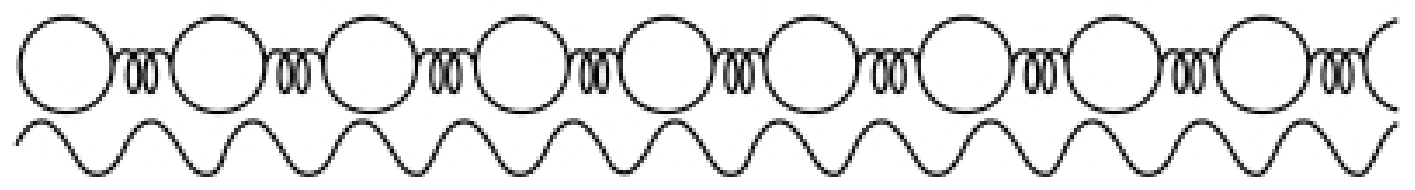

Figure 1.6. Charge desnsity waves. The charge density wave arises from an electronphonon interaction giving rise to the (a) commensurate state, and the incommensurate state (b). In the commensurate state, the potential is "locked" onto the lattice and pins the lattice. Conversely, the lattice can slide in the incommensurate state because the wavelength of the underlying potential is related to the atomic spacing by an irrational number. This gives rise to lattice distortions, thereby lowering the energy of the overall system. 


\subsection{DESCRIPTION OF LOW-TEMPERATURE SPECIFIC HEAT MEASUREMENTS}

Metals that can readily be obtained in high purity make a suitable choice for measurement standards for low-temperature specific heat. We elected to use a copper single crystal obtained from Alpha Aesar as a reference. The copper single crystal was 99.99999\% pure and was cut, oriented, and vacuum annealed prior to measurement. Specific-heat measurements were made on an apparatus designed around a ${ }^{3} \mathrm{He}$ insert capable of obtaining temperatures as low as $0.3 \mathrm{~K}$. A diagram of the apparatus is shown in Figure 1.7. An evaluation of the calorimetric performance was obtained by measuring the specific heat of copper from $T \approx 0.5 \mathrm{~K}$ to $T \approx 100 \mathrm{~K}$. The results presented here form the basis for the reporting of accuracy and precision for specific-heat measurements described in the following chapters.

\subsubsection{Semi-Adiabatic Pulse}

The specific heat of the empty calorimeter and sample stage is measured separately and subtracted from the specific heat to obtain the specific heat of the sample. A semiadiabatic pulse technique was used to measure the specific heat from the lowest temperature up to $10 \mathrm{~K}$, while an isothermal technique was used from $10 \mathrm{~K}$ to $100 \mathrm{~K}$. In the semiadiabatic method, the sample is thermally isolated from a constant temperature block. The block is set at a constant temperature while the sample is heated to several predetermined temperatures. A variable energy pulse is added to the sample, which brings it to the next temperature in the sequence. This process is repeated until the desired temperature range is covered, shown in Figure 1.8. A complete sampling of the entire temperature range is achieved by repeated measurements with decreasing $\Delta T$ values, in our measure- 


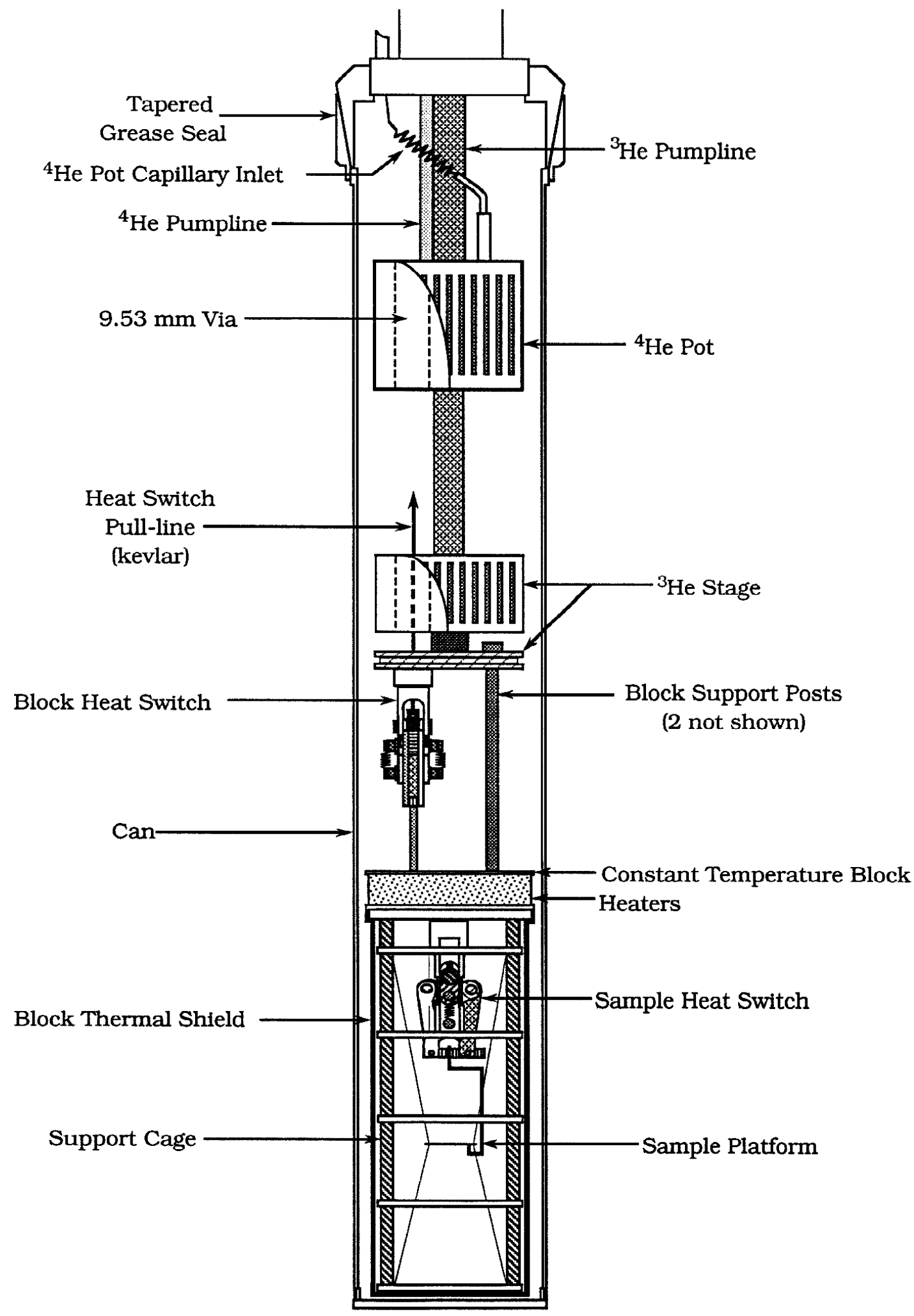

Figure 1.7. Semi-adiabatic apparatus for measuring $C_{p}$. 
ments these can be as low as $0.25 \mathrm{~K}$. Semi-adiabatic conditions are obtained by adding enough heat to obtain a flat drift at each temperature.

\subsubsection{Isothermal Technique}

In the isothermal method, the block is set at a temperature that lies between a warming drift (foredrift) and a cooling drift (afterdrift). After the foredrift and afterdrift data is collected, the block is heated to another predetermined temperature. This process is repeated until the desired temperature range is covered. The isothermal technique is illustrated in Figure 1.9. Thus, the isothermal technique differs from the semi-adiabatic

\section{Semi-Adiabatic Pulse}

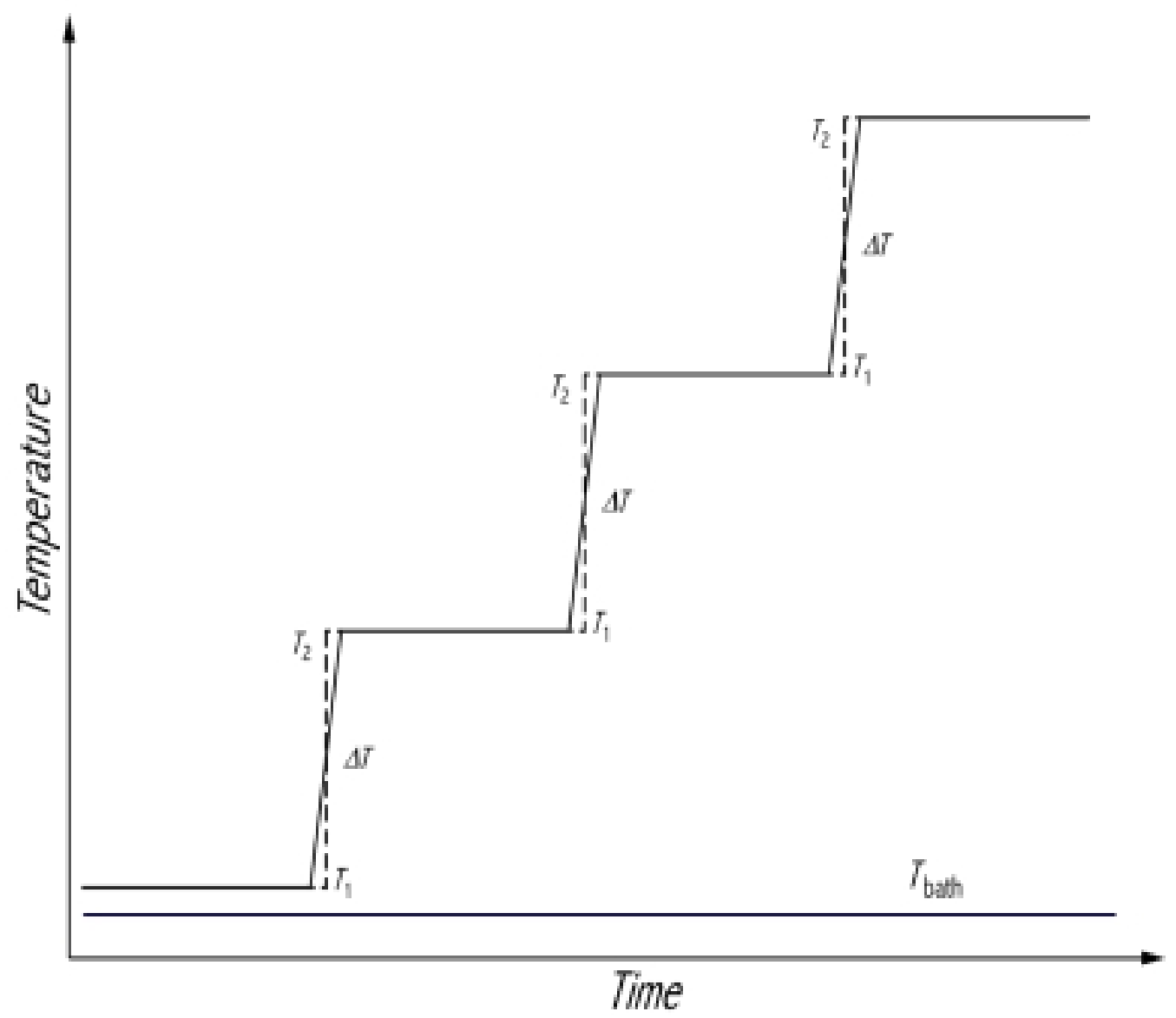

Figure 1.8. The semi-adiabatic pulse technique. 
pulse technique in that the block is set to a temperature that is between warming and cooling drifts, and no background heating is required.

\subsubsection{Calorimeter Performance}

Below $30 \mathrm{~K}$, the copper results were compared to the zero-field low-temperature data of Holste et al. [13] Martin [14], Phillips et. al. [15] and the high-temperature results of Martin [16]. A copper reference specific heat containing these zero-magnetic field results was made by averaging the data of Holste, Martin, and Phillips, and fitting the data to the expression

$$
C_{r e f}=\gamma T+\sum_{n} B_{2 n-1} T^{2 n-1} \quad\{n=1,3,4, \ldots, 8\}
$$

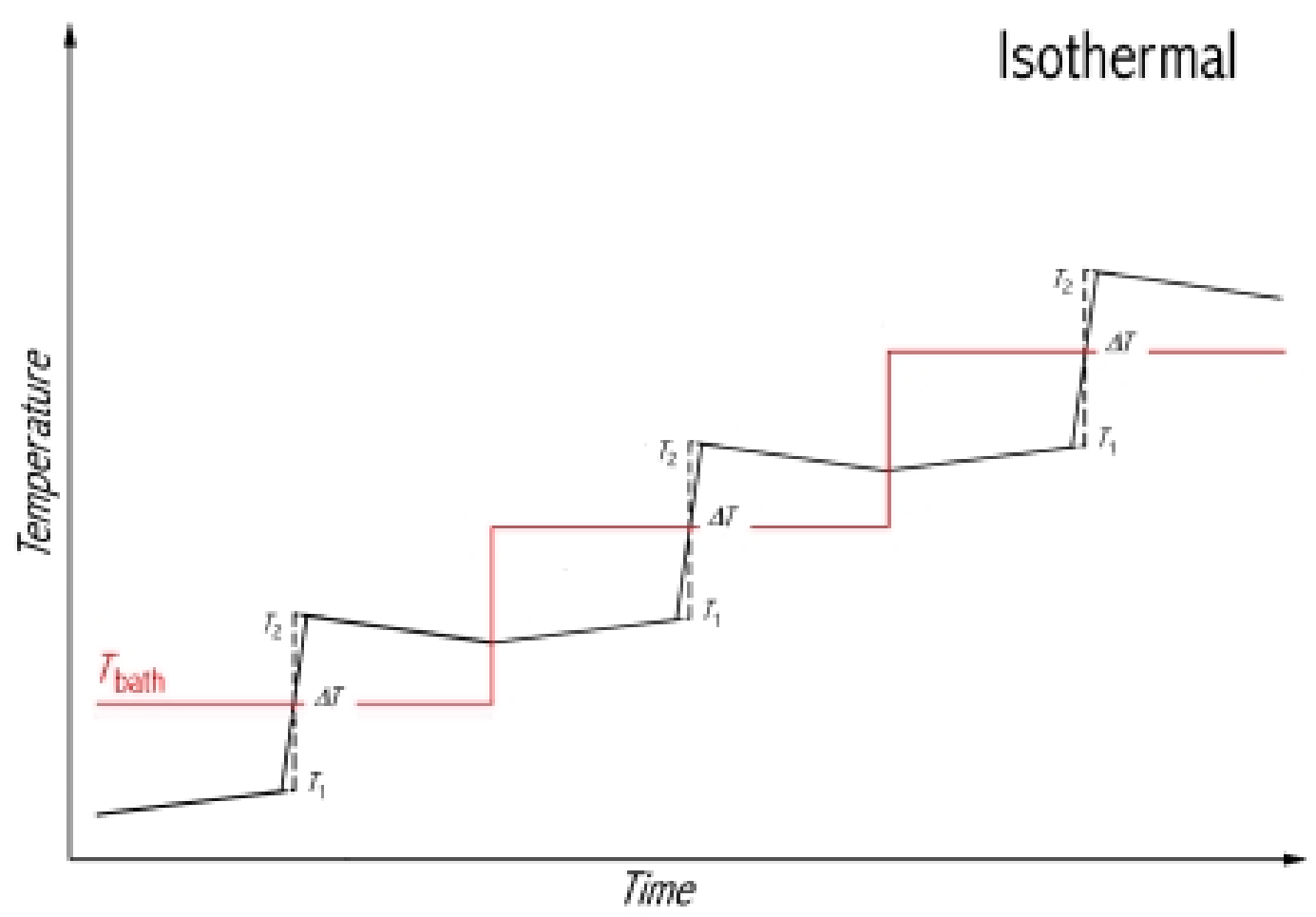

Figure 1.9. The isothermal technique. 
from $0.5 \mathrm{~K}$ to $50 \mathrm{~K}$. The root mean square deviation of the fit was $0.5 \%$. A comparison of the three low-temperature results are shown in Figure 1.10 from $0.5 \mathrm{~K}$ to $30 \mathrm{~K}$ as a deviation table where zero on the y-axis represents the average of these three measurements. Our low-temperature results were then compared against this reference function and are shown in Figure 1.11. Above $30 \mathrm{~K}$ our results were compared against the hightemperature measurements of Martin [16]. The high temperature results are plotted in Figure 1.12. Overall, our results fell within $\pm 0.5 \%$ of the scatter obtained from the

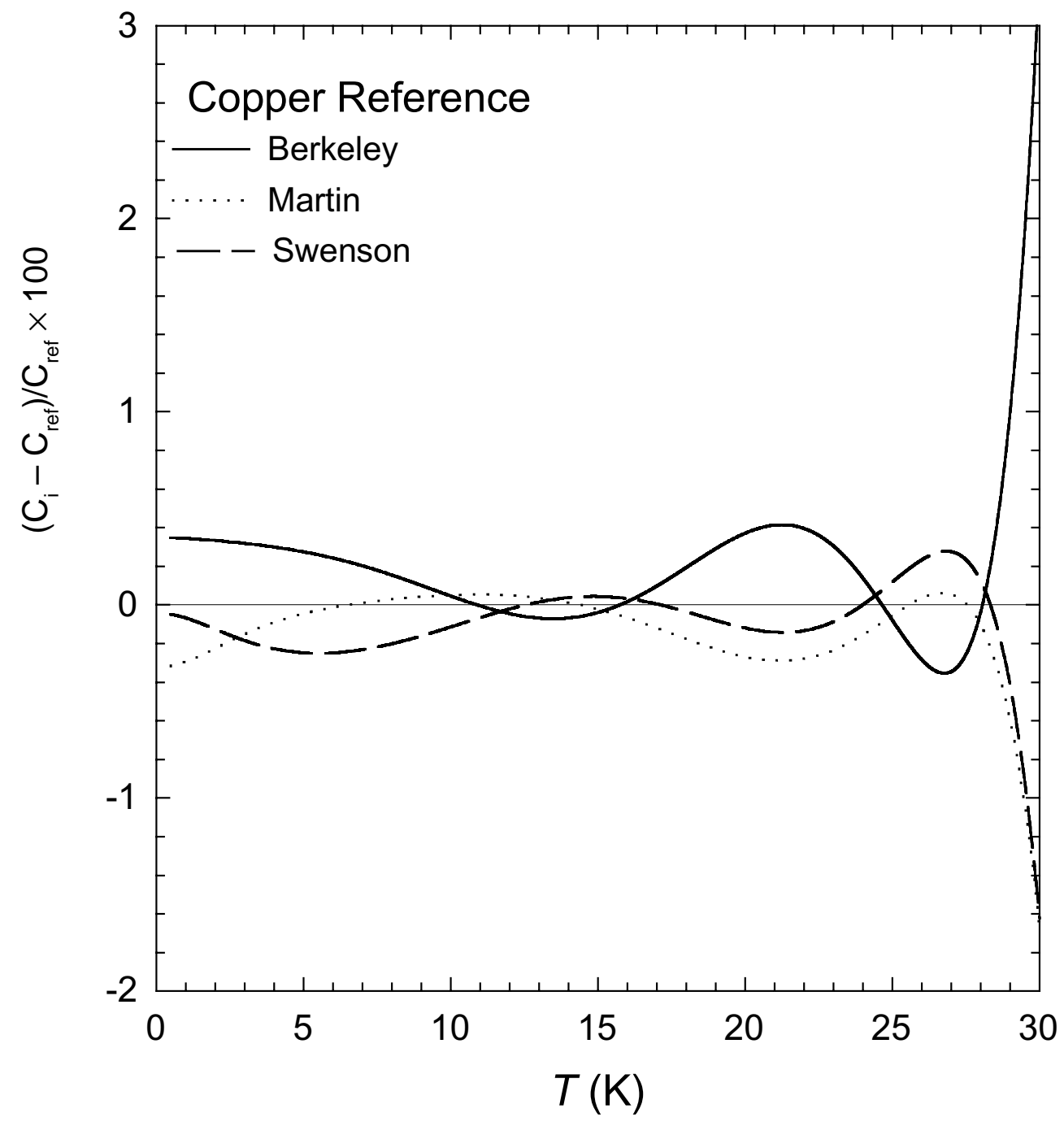

Figure 1.10. Calorimetric standard. 
reference measurements, and our resolution was better than $0.1 \%$. As low temperatures, our results are within the scatter of the three measurements considered by most to be the best measurements on copper. With these data, we are in a position to address the thermodynamics of phase changes that occur at low-temperature with a high degree of accuracy and precision.

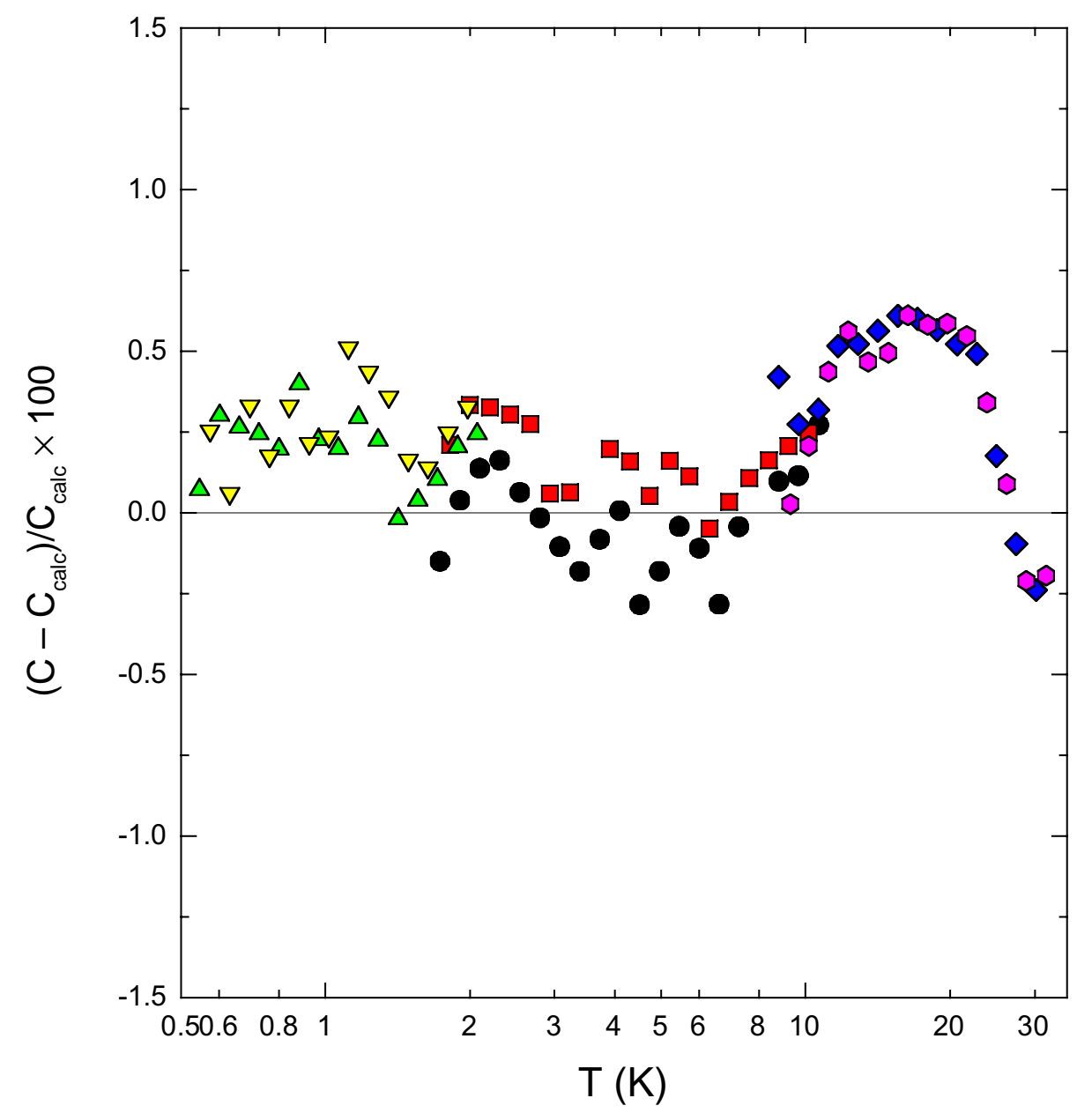

Figure 1.11. Deviation table. The scatter in our $C_{p}$ measurements is plotted against the average of the copper reference. One can see a systematic deviation that is $\pm 0.5 \%$ for all temperatures and our precision is better than $0.1 \%$. The different data symbols signify different $C_{p}$ runs over different $\Delta T$ intervals. 


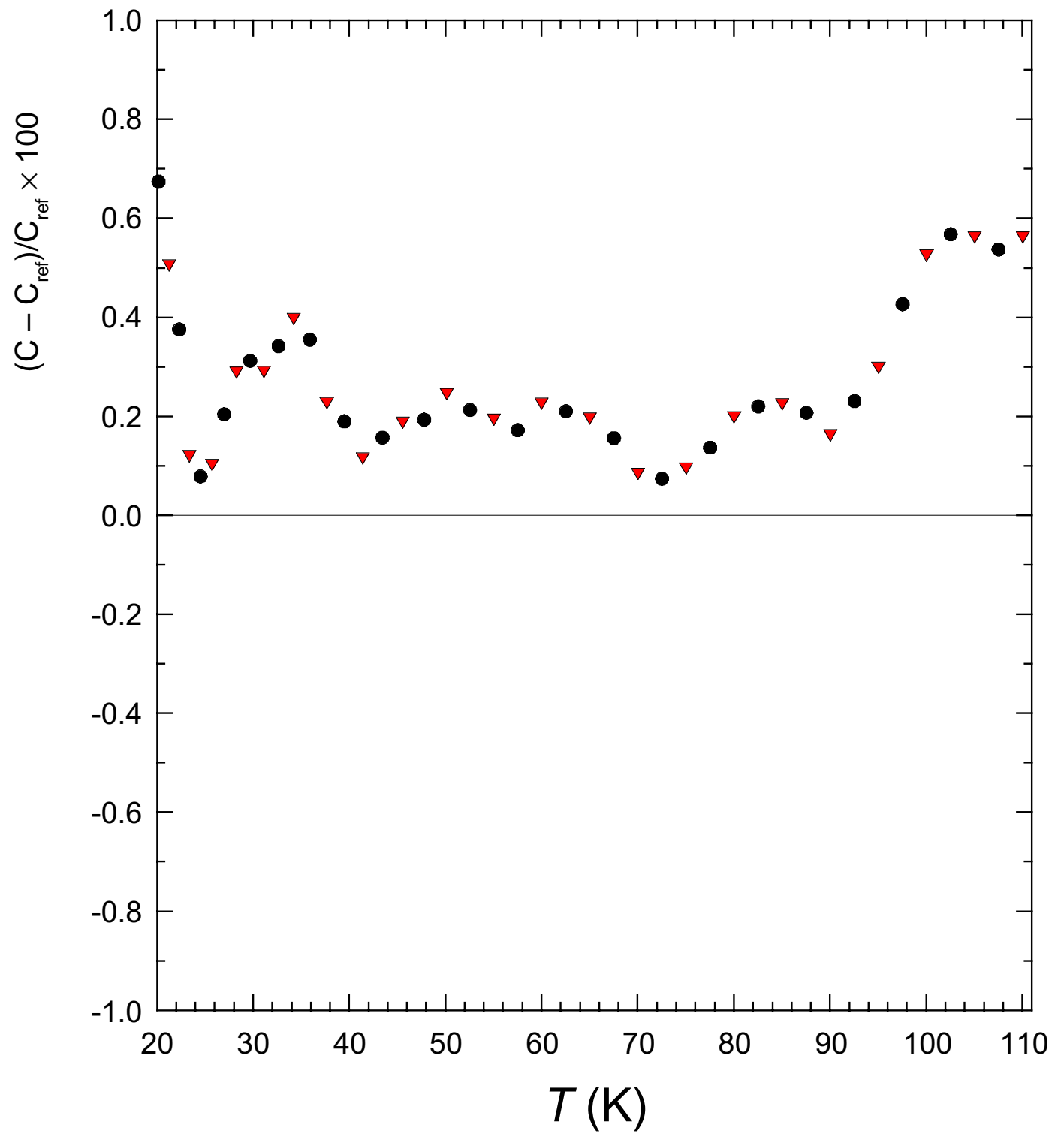

Figure 1.12. The error in our measurements relative to the copper reference file is shown from $20 \mathrm{~K}$ to $110 \mathrm{~K}$. The error over the entire range is $\pm 0.5 \%$ with slightly better results from $40 \mathrm{~K}$ to $100 \mathrm{~K}$. 


\section{REFERENCES}

[1] S. Sachdev, "Quantum Phase Transitions" Cambridge University Press (Cambridge, 1999).

[2] N. D. Mathur, Nature 394, 39 (1998).

[3] S. S. Saxena, P. Agarwal, K. Ahlin, F. M. Grosche, R. K. W. Haselwimmer, M. J. Steiner, E. Puch, I. R. Walker, S. R. Julian, P. Monthoux, G. G. Lonzarich, A. Huxley, I. Sheikin, D. Braithwaite, and J. Flouquet, Nature 406, 587 (2000).

[4] J. Weertman, and J.R. Weertman, Elementary Dislocation Theory (Copyright 1992, Oxford Unversity Press, Inc., London), pp. 15-22.

[5] D. Wallace, Thermodynamics of Crystals (Copyright 1998, Dover Publications, Inc., New York), pp. 3-9.

[6] J. P. Wolfe, Imaging Phonons (Copyright 1998, Cambridge University Press, Inc., London), p. 25.

[7] J. G. Byrne, Recovery Recrystallization and Grain Growth (Copyright 1965, The Macmillan Company, Toronto, Ontario, Canada), pp. 1-17.

[8] J. B. Ott and J. Boerio-Goates, Chemical Thermodynamics: Principles and Applications, Vol.1 (Copyright 2000, Academic Press, London), pp. 231-240.

[9] J. Honig, Thermodynamics (Second edition, Copyright 1999, Academic Press, London), pp. 147-149.

[10] E. S. R. Gopal, Specific Heats at Low Temperatures (Copyright 1966, Plenum Press, New York), pp. 1-80.

[11] C. Kittel, Introduction to Solid State Physics (seventh edition, Copyright 1996, John Wiley \& Sons, New York), pp. 130-150.

[12] A. W. Overhauser, Adv. Phys., 27, 343, (1978).

[13] J. C. Holste, T. C. Cetas, and C. A. Swenson, Rev. Sci. Instrum. 43, 670 (1972).

[14] D. L. Martin, Phys. Rev. B. 8, 5357 (1973).

[15] N. E. Phillips, J. P. Emerson, R. A. Fisher, J. E. Gordon, B. F. Woodfield, and D. A. Wright, unpublished data.

[16] D. L. Martin, Rev. Sci. Instrum. 58, 639 (1987). 


\title{
ELASTIC AND THERMODYNAMIC PROPERTIES
} OF SINGLE-CRYSTAL AuZn NEAR THE MARTENSITIC TRANSITION

\author{
How should we recognize a martensitic transformation \\ if it bumped into us on a dark \& austy night? \\ - P. C. Clapp
}

\author{
2.1 ABSTRACT \\ 2.2 INTRODUCTION \\ 2.3 SAMPLES \\ 2.4 MEASUREMENTS \\ 2.4.1 Resonant Ultrasound Spectroscopy \\ 2.4.2 Semi-Adiabatic Calorimetry \\ 2.5 RESULTS \\ 2.5.1 Elasticity \\ 2.5.2 Specific Heat \\ 2.6 DISCUSSION \\ ACKNOWLEDGMENTS \\ REFERENCES
}

\subsection{ABSTRACT}

The elastic shear moduli of the B2 cubic ordered alloy AuZn as a function of temperature, and the specific heat from $T \approx 0.6 \mathrm{~K}$ to $T \approx 100 \mathrm{~K}$ is presented in this chapter. Elasticity and specific-heat measurements were made on single-crystal samples using resonant ultrasound spectroscopy (RUS) and semi-adiabatic calorimetry, respectively. 
This material exhibits a continuous ${ }^{1}$ phase transition at $64.75 \mathrm{~K}$, and analysis of the specific-heat data give values for the entropy of transition $\left(\Delta S_{t r}\right)$ of $2.02 \mathrm{~J} \mathrm{~K}^{-1} \mathrm{~mol}^{-1}$, electronic specific-heat $(\gamma) 0.38 \mathrm{~mJ} \mathrm{~mol}^{-1} \mathrm{~K}^{-1}$ and low-temperature limiting Debye temperature $\left(\Theta_{D}\right) 219 \mathrm{~K}$. The shear moduli change approximately linearly with temperature above the transition, with $c^{*}=\left[0.50\left(c_{11}-c_{12}\right)\right]$ displaying an anomalous (positive) coefficient. This alloy displays a large elastic shear anisotropy $c_{44} \approx 7$ at $300 \mathrm{~K}$, increasing to $\approx$ 9 near the phase transition. Calculating $\Theta_{D}$ from an extrapolation of the high-temperature phase elastic constants to $T=0 \mathrm{~K}$ yields a value of $207 \mathrm{~K}$, in favorable agreement with the thermal value, despite the intervening phase transition. A mechanical test performed at liquid helium temperatures verify that AuZn exhibits the shape memory effect. To this end, the martensitic transformation temperature in AuZn is the lowest observed for a shape-memory alloy.

\subsection{INTRODUCTION}

The gold-zinc system displays a congruent melting point near equal atomic composition, which permits single crystals of the ordered $\mathrm{B} 2(\mathrm{CsCl}$ structure) phase to be readily prepared. It is well-known that AuZn displays a phase transformation at $64 \mathrm{~K}$ although there is little published work on the fundamental thermodynamic properties of the phase transformation. At a martensitic transition a polycrystal of the martensite structure forms, usually with strict twinning relationships between crystallites, via small, reversible atomic displacements. For this reason the shape memory effect is linked to the martensitic transition.

${ }^{1}$ Herein we use "continuous" to replace the older Ehrenfest second-order classification. 
The phase transformation in AuZn has been described as martensitic with the martensite structure determined as trigonal (P3) symmetry [1]. This experimental result is supported by the observation that certain alloys with the B2 structure display a large elastic shear modulus anisotropy, $A=c_{44} / c^{*}$ and through Zener's [2] connection of this observation to a lattice instability, a martensitic transformation is expected in AuZn promoted by a weak resistance to $c^{*}=0.50\left(c_{11^{-}} c_{12}\right)$ shears. The $\mathrm{B} 2$ cubic lattice is the structure of many shape-memory alloys such as $\mathrm{AuCd}$, TiNi, and other well studied martensites such as $\mathrm{Ni}_{\mathrm{x}} \mathrm{Al}_{1-\mathrm{x}}$ for $\{0.60<\mathrm{x}<0.65\}$ [3]. These $\mathrm{B} 2$ martensites all display an anomalous temperature coefficient for the soft shear modulus, $c^{*}$, indicating that even at several hundred degrees above the transition, the structure has little resistance to deformations associated with $c^{*}$, and this resistance to deformation decreases with decreasing temperature. Consequently, this softness may even permit some fraction of the crystal to explore other metastable phases above the transition, perhaps leading to some disorder in the lattice. The elastic moduli are sensitive to changes in the free energy, involving lattice strains, and may reveal disorder or glass-like behavior at low temperatures. Thus, the relationship between entropy and structural properties coupled with the lack of low-temperature thermodynamic data has motivated us to perform a detailed study of the elastic and thermodynamic properties of the B2 cubic material AuZn.

\subsection{SAMPLES}

Elemental $\mathrm{Au}$ and $\mathrm{Zn}$ metals in equal atomic composition, having purity of 99.9 at. \% and 99.99 at. \% respectively, were sealed in an evacuated quartz tube. Upon achieving a vacuum level less than $1 \mathrm{~Pa}$, we sealed the quartz tube by pulling two spiral turns. The 
inner quartz diameter was less than $1 \mathrm{~mm}$ in the spiral section. The configuration was heated to $1373 \mathrm{~K}$ to obtain the melt of the AuZn phase and shaken for homogenization. The quartz spiral end was submerged in water for cooling. Directional solidification through the spiral extinguished all but one grain, permitting the bulk of the sample to solidify as a single crystal. The crystallographic orientation of the large grain was determined using back-reflection Laue x-ray diffraction.

Several parallelepipeds $([100] \times[010] \times[001])$ were cut using a diamond wafer saw and polished using $\mathrm{SiC}$ papers and diamond lapping films to $1 \mu \mathrm{m}$. Further Laue studies showed a disappearance of the reflection patterns, indicating that substantial surface damage resulted from cutting and polishing.

In order to reduce this damage, the samples were re-sealed in quartz tubing with an Ar atmosphere and annealed at $673 \mathrm{~K}$ for 120 hours. The Laue patterns of the annealed samples showed that the damage effect was largely eliminated, and acceptable singlecrystal samples were obtained. The same sample was used in both the elasticity and specific-heat measurements. The weight of the parallelepiped sample was $96.5 \mathrm{mg}$.

\subsection{MEASUREMENTS}

\subsubsection{Resonant Ultrasound Spectroscopy}

Resonant ultrasound spectroscopy (RUS) [4] is a technique in which the complete elastic tensor is determined from the spectrum of the mechanical resonances of an object. This measurement is made with the rectangular parallelepiped sample that is vertically supported across a body diagonal between two piezoelectric transducers. One transducer is driven by a sinusoidal voltage that is swept over a suitable frequency range, and the 
response of the object is detected by a second transducer. A heterodyne receiver technique is employed to produce the final spectrum that contains amplitude and phase information. A commercial RUS unit was used for this measurement [5]. A numerical procedure is used to generate the expected frequency spectrum for a given set of elastic moduli, $c_{\mathrm{ij}}$, and comparison with data of known crystal symmetries and orientations provides the matrix $c_{\mathrm{ij}}$, consistent with the measurement. The details of this technique are available elsewhere [6]. The only contact between sample and transducers is at corner points, and the surfaces are unconstrained so a single measurement may sweep through the transition despite surface relief developing at the transition.

\subsubsection{Semi-Adiabatic Calorimetry}

The specific heat was measured in zero magnetic field using an apparatus designed around $\mathrm{a}^{3} \mathrm{He}$ insert capable of attaining temperatures as low as $0.4 \mathrm{~K}$. The sample was thermally attached to the sample platform with a thin layer of Apiezon $\mathrm{N}$ grease. The specific heat of the empty sample platform was measured separately. The specific heat of the empty sample platform and the specific heat of Apiezon N [7] were subtracted from the total specific heat in order to obtain the specific heat of the sample. Below $10 \mathrm{~K}$ the sample, weighing only $96.5 \mathrm{mg}$, contributed $2 \%$ to the total specific heat, and $12 \%$ at the peak of the transition. The performance of the calorimeter was tested by measuring a single crystal of copper metal with a chemical purity of 99.99999 at. \%. Below $30 \mathrm{~K}$, the copper results were compared to zero-field low temperature data of Swenson [8], Martin [9], and Phillips [10], and above $30 \mathrm{~K}$ the results were compared to the high-temperature measurements of Martin [11]. Our results fell within $0.25 \%$ of the scatter obtained from the reference measurements, and our resolution was better than $0.1 \%$. 
A semi-adiabatic pulse technique was used to measure the specific heat from the lowest temperature up to $10 \mathrm{~K}$, and this technique was also used to map the martensitic transition from $T_{1}=45 \mathrm{~K}$ to $T_{2}=70 \mathrm{~K}$. In the semi-adiabatic method, the sample is thermally isolated from a constant temperature block. The block is set at a constant temperature while the sample is heated to a sequence of predetermined temperatures. An energy pulse is added to the sample to bring it to the next temperature. This process is repeated until the desired temperature range is covered. A complete sampling of the entire temperature range is achieved by repeated measurements with decreasing $\Delta T$ values, in our measurements as low as $0.25 \mathrm{~K}$. Semi-adiabatic conditions are obtained by adding enough heat to obtain a flat drift at each temperature.

An isothermal technique was used in the temperature region $10 \mathrm{~K}$ to $100 \mathrm{~K}$. In the isothermal method, the block is set at a temperature that lies between a warming drift (foredrift) and a cooling drift (afterdrift). After the foredrift and afterdrift data is collected, the block is heated to another predetermined temperature. This process is repeated until the desired temperature range is covered. Thus, the isothermal technique differs from the semi-adiabatic pulse technique in that the block is set to a temperature that is between warming and cooling drifts. The details of this apparatus and thermometry are published elsewhere [12], but a description of a similar apparatus can be found in a separate account [13]. 


\subsection{RESULTS}

\subsubsection{Elasticity}

A plot of the temperature dependence of the sample resonance frequencies is shown in Figure 2.1. The frequencies of these modes are largely determined by the $c^{*}$ shear modulus, as it is the smallest elastic constant in the system by nearly an order of magnitude. Resonances containing information about the longitudinal modulus occur above $1 \mathrm{MHz}$, where the high density of states is beyond our present modeling capability, and reliable identification of mode type is not possible. The transition at $64 \mathrm{~K}$ occurs without significant precursor changes when approaching the transition temperature $T_{\mathrm{M}}$ from

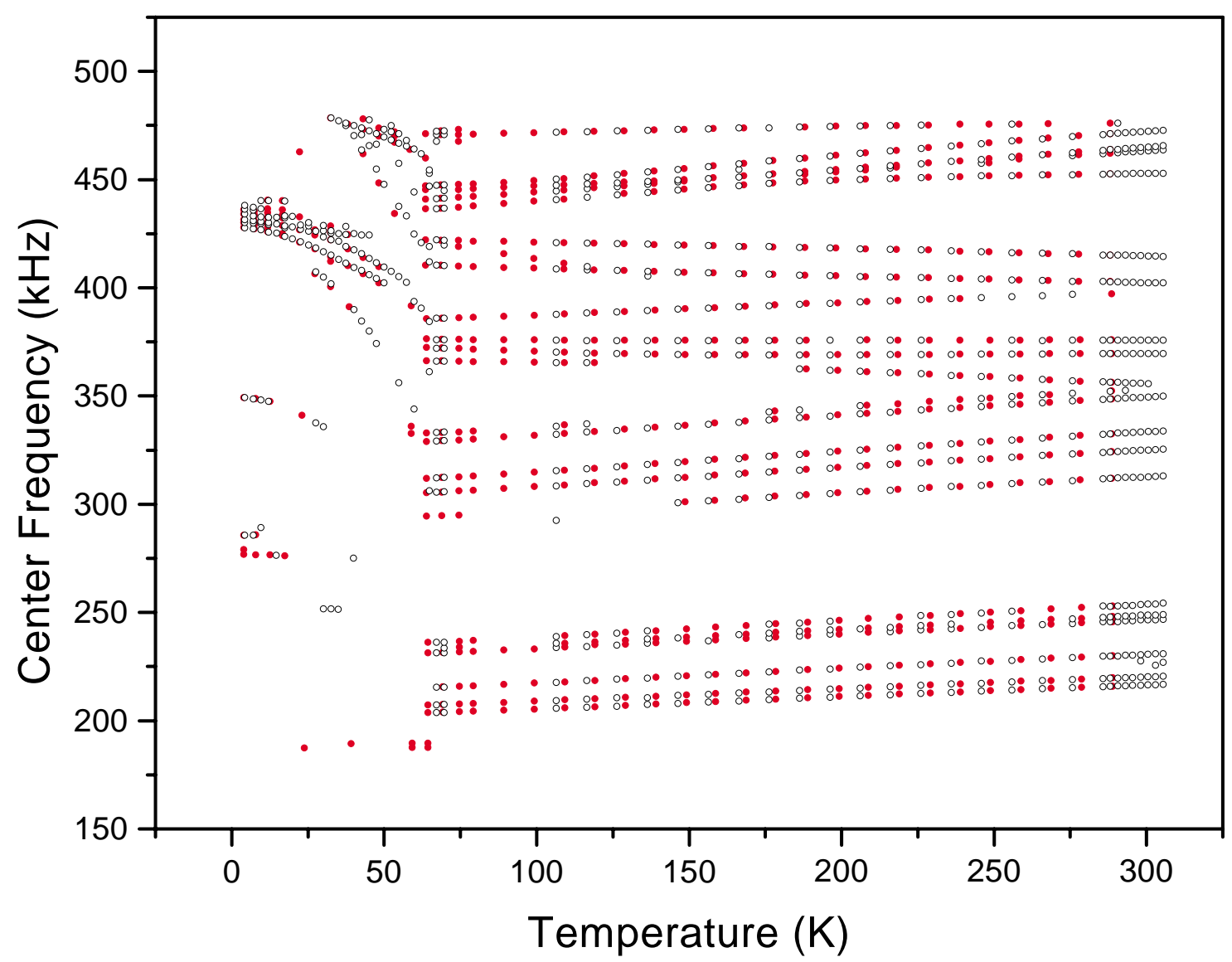

Figure 2.1. Resonance frequencies for single-crystal AuZn as a function of temperature. The open circles show the cooling curves while the filled circles represent the warming curves. 
above (e.g., a soft mode). This result contrasts with measurements made on a large-grainsize polycrystal sample, Figure 2.2, where curvature appears as the transition is approached. This curvature may be attributed to strains generated by stresses evolved at the grain boundaries that modify the free energy near the transition. After cycling from $300 \mathrm{~K}$ to $4 \mathrm{~K}$ and back to $300 \mathrm{~K}$, the resonances return to the same frequencies, indicating that the sample returns to an identical elastic state after passing through the transition. The widths of the resonance lines are proportional to the internal friction, and they become large below $T_{\mathrm{M}}$. Correspondingly, the amplitudes diminish making the peaks much harder to identify below $T_{\mathrm{M}}$. This plot contains data from a complete cooling/heating cycle.

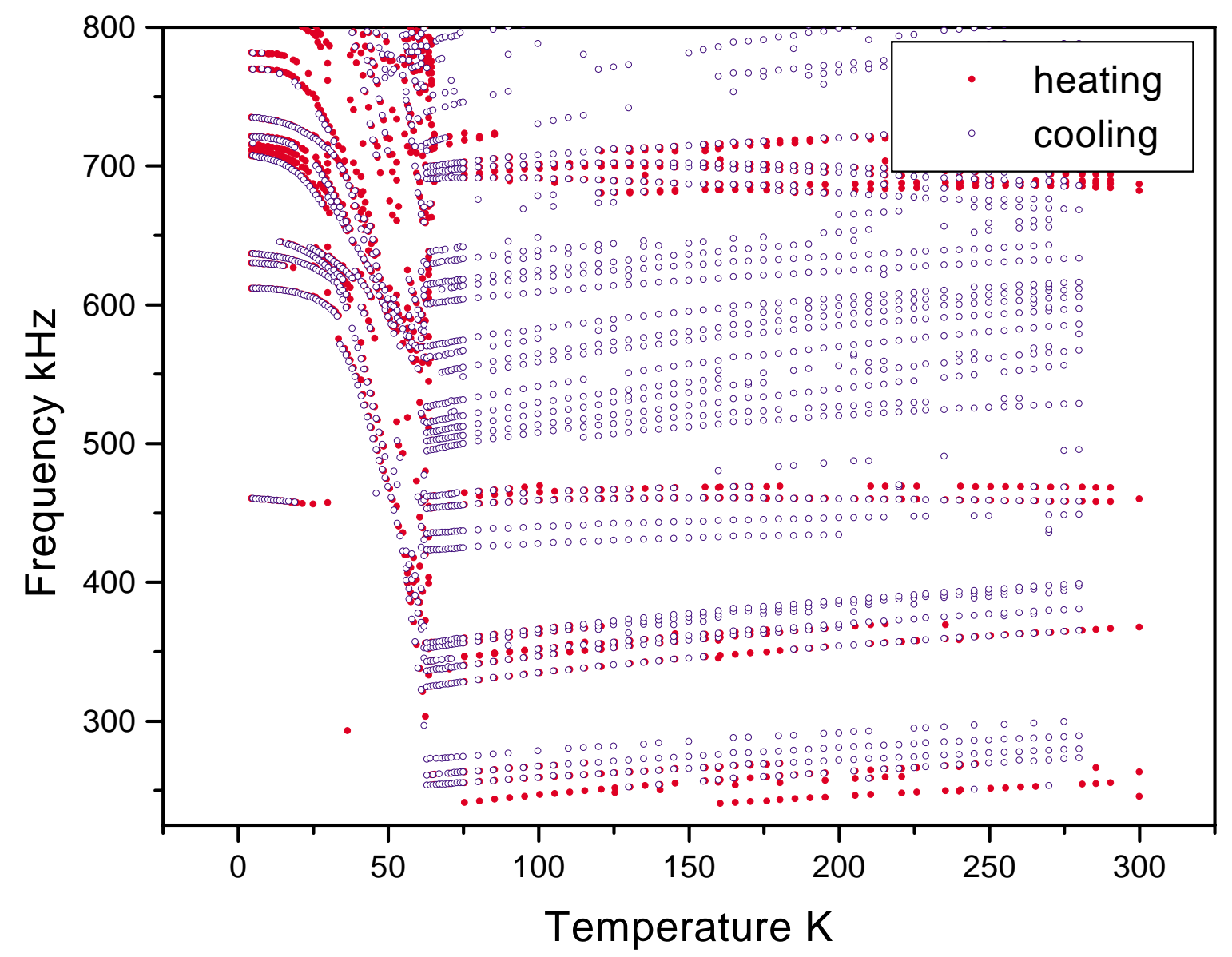

Figure 2.2. Resonance frequencies for large grain polycrystal AuZn as a function of temperature. 
Figure 2.3 shows details of the low temperature behavior of the peak frequencies and typical widths in the polycrystal. The dissipation is not a maximum at $T_{\mathrm{M}}$ but in the temperature region of $42 \mathrm{~K}$ to $60 \mathrm{~K}$. In this region mobile twin boundaries in the martensite may shift, absorbing the energy of the ultrasonic strains. Below $10 \mathrm{~K}$ the peaks are sharp, but we were unable to obtain a consistent elastic fit to any symmetry. This result suggests that the sample displays nonuniform symmetry through its volume, on a length scale comparable with the wavelength of the acoustic standing wave. The behavior below the transition does tell us that the smallest modulus has approximately doubled, and there are no anomalous temperature coefficients. The transition occurs at $64 \mathrm{~K}$ with $1 \mathrm{~K}$ ( $\pm 1 \mathrm{~K}$ ) of hysteresis. We have determined the single-crystal elastic shear moduli for the

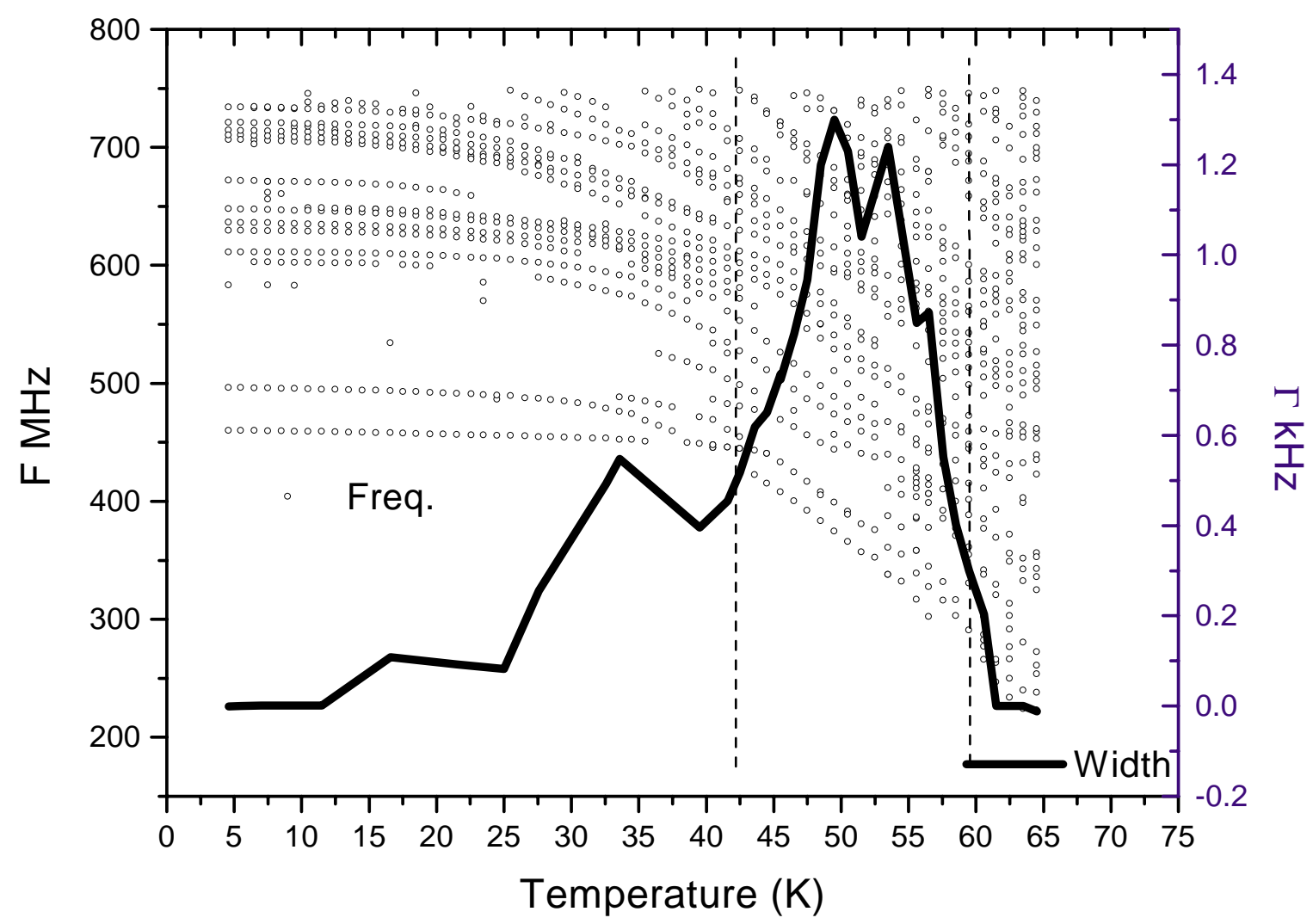

Figure 2.3. Resonance frequencies and typical peak width (internal friction) below $T_{\mathrm{M}}$ 
temperature range above the transition. A linear fit to the moduli and anisotropy yields

$$
\begin{aligned}
& c_{44}=66.6( \pm 0.2)-0.037( \pm 0.001) T, \\
& c^{*}=6.66( \pm 0.01)+0.0045( \pm 0.0001) T ; \text { and } \\
& A=9.80( \pm 0.03)-0.0097( \pm 0.0002) T .
\end{aligned}
$$

An expression for the long-wavelength Debye temperature involving only the shear moduli allows us to calculate a temperature of $207 \mathrm{~K}$ from the $T=0$ linear extrapolation of the moduli. The data in Figure 2.4 shows the cubic shear elastic moduli and also the shear anisotropy, which varies from 7 at room temperature to 9 near the transition. The soft shear modulus, $c^{*}$, displays an anomalous positive temperature dependence. The deviations at $\sim 125 \mathrm{~K}$ and $\sim 183 \mathrm{~K}$, visible most clearly in $c_{44}$ are outside our estimated

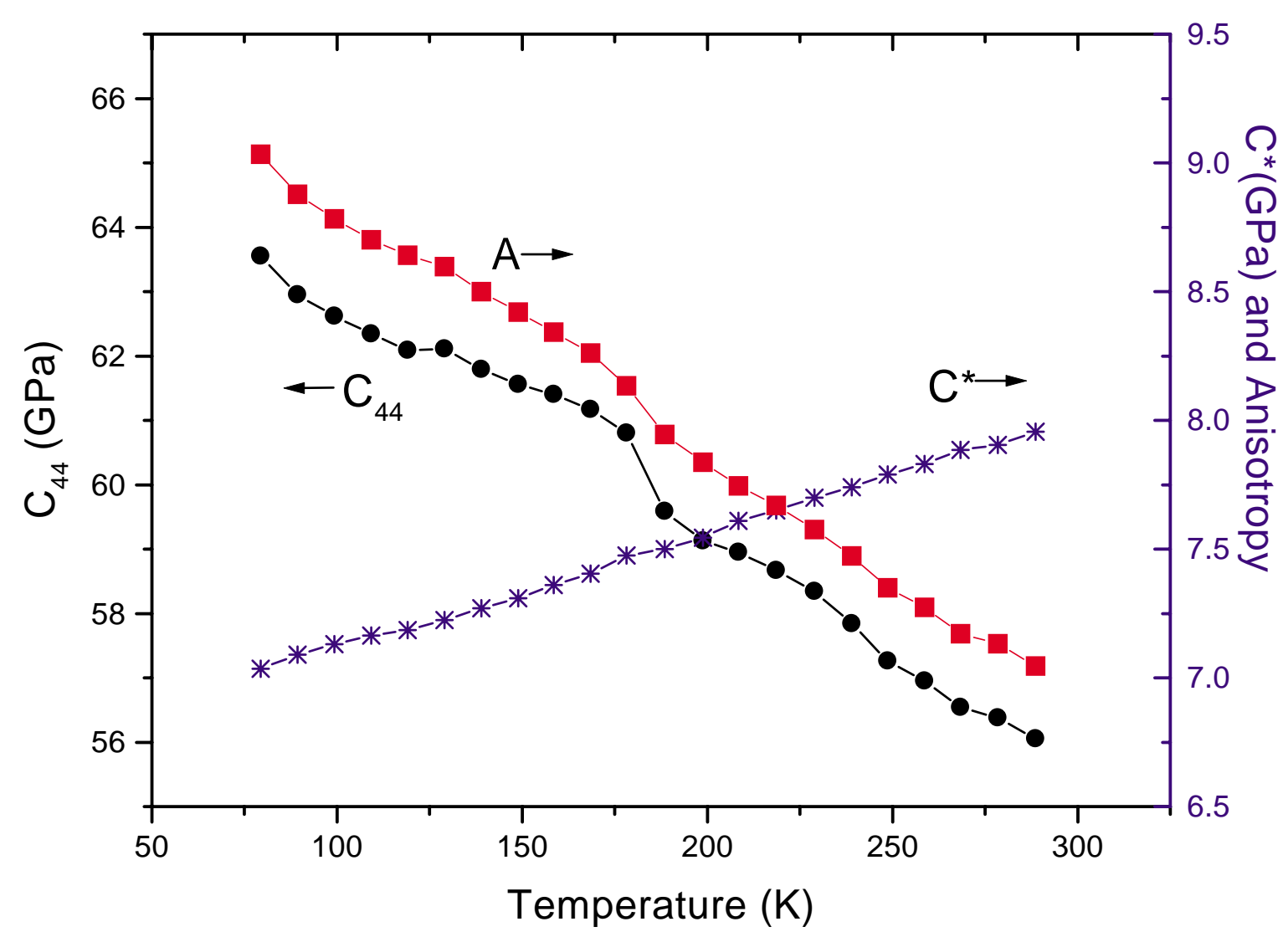

Figure 2.4. The data is fitted to the shear moduli and anisotropy. 
error bars. These deviations may be associated with the transition in the form of a metastable state or as portions of the sample transforming early as a result of an inhomogeneous composition. Table 2.1 contains values of the moduli, with estimated errors, at selected temperatures. These values agree well with published pulse-echo ultrasound measurements [14]. While our measured RUS peaks are dominated by the shear moduli, the overdetermination of the parameters applies strong self-consistency constraints.

Allowing the program to attempt to fit $c_{11}$ and $c_{12}$ produces values some $10 \%$ lower than the pulse echo data [15] and also a positive temperature coefficient for $c_{11}$. Nonetheless, these data provide a bulk modulus which is in good agreement with a recent pressure-cell neutron scattering value of $122 \mathrm{GPa}[16]$.

\subsubsection{Specific Heat}

The specific heat measured in zero magnetic field of the AuZn martensitic transition is shown in Figure 2.5. In this figure, the lattice specific heat has been subtracted from

Table 2.1. Elastic moduli with estimated errors for single-crystal AuZn

\begin{tabular}{|c|c|c|c|}
\hline $\begin{array}{c}T(\mathrm{~K}) \\
{[( \pm 1 \mathrm{~K})]}\end{array}$ & $\begin{array}{c}c_{44}(\mathrm{GPa}) \\
[ \pm 0.8)]\end{array}$ & $\begin{array}{c}c^{*}(\mathrm{GPa}) \\
{[( \pm 0.2)]}\end{array}$ & $\begin{array}{c}A \\
{[( \pm 0.3)]}\end{array}$ \\
\hline 79.4 & 63.55 & 7.04 & 9.03 \\
\hline 99.3 & 62.62 & 7.13 & 8.78 \\
\hline 119.1 & 62.09 & 7.19 & 8.64 \\
\hline 139.0 & 61.79 & 7.27 & 8.50 \\
\hline 158.5 & 61.40 & 7.36 & 8.34 \\
\hline 178.2 & 60.80 & 7.48 & 8.13 \\
\hline 198.8 & 59.13 & 7.55 & 7.84 \\
\hline 218.6 & 58.67 & 7.65 & 7.67 \\
\hline 239.0 & 57.84 & 7.74 & 7.47 \\
\hline 258.6 & 56.95 & 7.83 & 7.27 \\
\hline 278.4 & 56.38 & 7.91 & 7.13 \\
\hline 288.6 & 56.05 & 7.96 & 7.05 \\
\hline
\end{tabular}




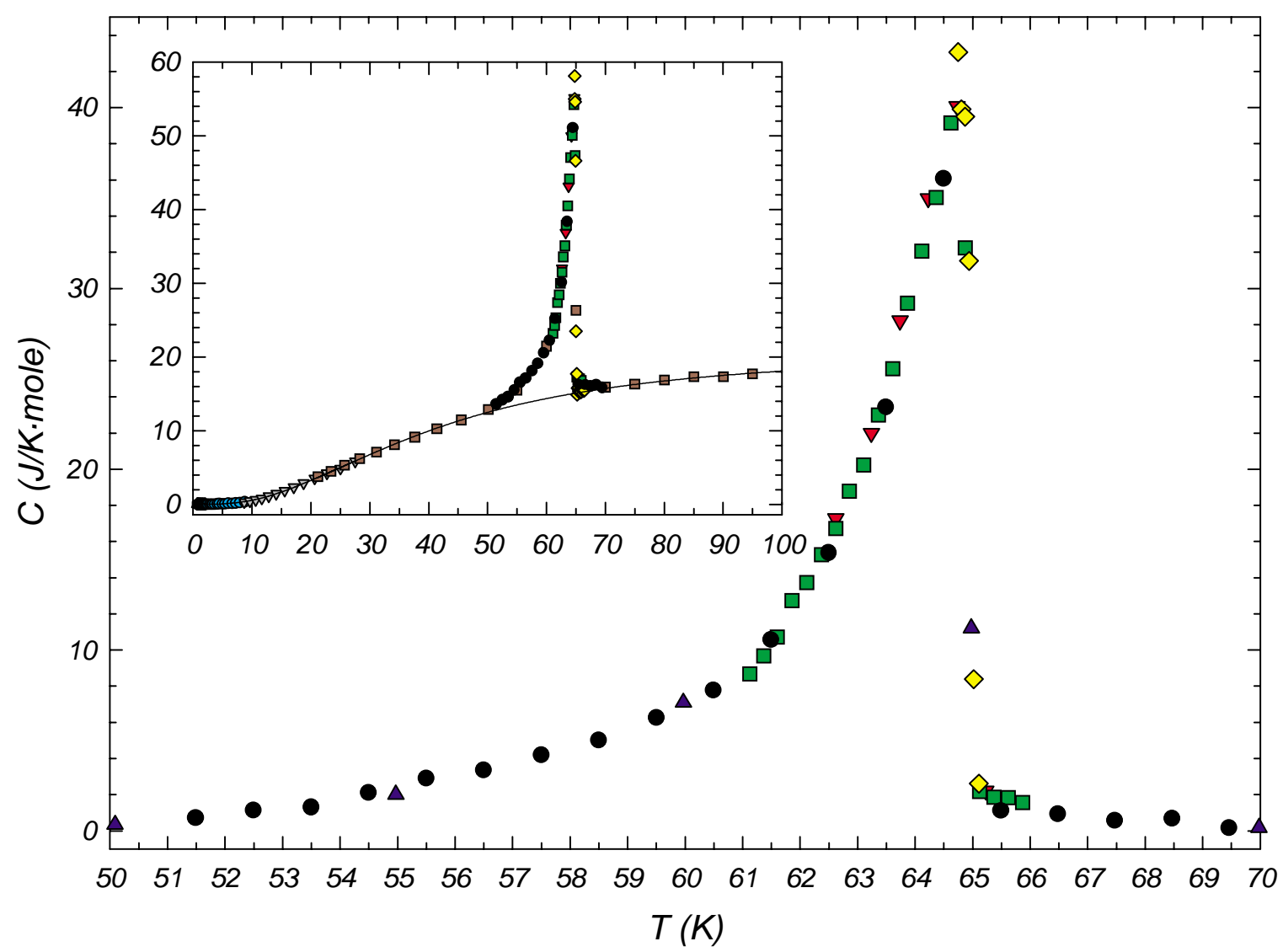

Figure 2.5. The specific heat of the martensitic transition with the lattice contribution subtracted. Inset: The fit of the lattice specific heat to the martensite and austenite phases. This fit was interpolated through the transition, and the different data symbols represent different runs with smaller $\Delta T$ values.

the total specific heat in order to show the shape of the transition. The transition at 64.75 $\mathrm{K}$ is clearly a continuous transition that is similar in shape to the transition that occurs in $\beta$-brass, another B2 alloy [17].

The electronic term $(\gamma)$ and the Debye temperature $\left(\Theta_{D}\right)$ were obtained from a fit to the low-temperature expansion of the Debye function given in Equation (2.1):

$$
C_{\mathrm{V}}=\gamma T+\mathrm{B}_{3} T^{3}+\mathrm{B}_{5} T^{5}
$$

where the required number of adjustable parameters for the lattice $\left(\mathrm{B}_{3}, \mathrm{~B}_{5}, \ldots ., \mathrm{B}_{\mathrm{n}}\right)$ increases at high temperatures. The electronic and lattice specific heat was fitted from 
$T=0.6 \mathrm{~K}$ to $T=10 \mathrm{~K}$. From this fit a Debye temperature of $219 \mathrm{~K}$ was obtained, and an electronic specific heat of $0.38 \mathrm{~mJ} \mathrm{~K}^{-2} \mathrm{~mol}^{-1}$ was obtained. In order to estimate the lattice specific heat at higher temperature, $\gamma$ and $\Theta_{D}$, obtained from the fit to (1), were substituted into a Debye-Einstein function, given in Equation (2.2) [18]

$$
C_{\mathrm{p}}=\left(3 \mathrm{R}\left[m D\left(\Theta_{D} / T\right)+n E\left(\Theta_{E} / T\right)\right]+\gamma T\right)
$$

where $m, n$, and $\Theta_{\mathrm{E}}$ are adjustable parameters; whereas $\Theta_{D}$ and $\gamma$ were fixed at the values obtained from the low-temperature fit to (1). The fit of (2) to the specific-heat data is shown in the inset of 2.5. The fit to the lattice was interpolated through the transition, and integration of $C / T$ from $T_{1}=47.5 \mathrm{~K}$ to $T_{2}=67.0 \mathrm{~K}$ gives an entropy of transition $2.02 \mathrm{~J} \mathrm{~K}^{-1} \mathrm{~mol}^{-1}$. Six runs were made through the transition, each using a smaller $\Delta \mathrm{T}$ indicated by a different data symbol in Figure 2.5. Between each run, the sample was cooled at a rate of $5 \mathrm{~K}_{\text {hour }}^{-1}$ to ensure reproducibility in the specific-heat data through the transition.

\subsection{DISCUSSION}

The linear, positive temperature coefficient of the $c^{*}$ elastic modulus in the austenite phase seems to be that of an impending proper ferroelastic transformation [19], but the low temperature behavior fails to match that of a simple model. Based on the RUS data shown in Figure 4.2, AuZn is likely to display thermoelastic behavior as the elastic constants in the austenite phase return to the same value after thermal cycling through the transition. From the specific-heat data, $\Theta_{D}$ does not change significantly because the lattice specific heat, shown in the inset of Figure 2.5, does not change significantly when going from the martensite to the austenite phase. In copper-based alloys it has been established that the vibrational properties of the austenite phase can be described by a 
Debye model with a fraction of modes represented by an Einstein term, while a Debye term is necessary for the martensite phase [20].

Despite the traditional thermodynamic description of martensitic transformations as first-order, this material exhibits a continuous transition that is similar in shape to that of the order-disorder transition in $\beta$-brass [21]. The specific-heat shown in Figure 2.5 tends to infinity at $T_{\mathrm{M}}$ with a characteristic lambda shape. Many shape-memory alloys such as NiTi, exhibit transformations accompanied by an isothermal latent heat contribution and by a large temperature hysteresis. A calculation of the entropy change in the order-disorder $\beta$-brass transition gives $\Delta S_{\mathrm{tr}}=3 \mathrm{~J} \mathrm{~K}^{-1} \mathrm{~mol}^{-1}$, or equivantly $\left(\Delta S_{\mathrm{tr}}=0.52 R \operatorname{Ln}(2)\right)$, a value that is larger than the $\left(\Delta S_{\mathrm{tr}}=0.33 R \operatorname{Ln}(2)\right)$ value that we report for AuZn. Presumably, the contributions to the entropy term are composed of a change in crystal symmetry with an associated phonon density of state entropy change.

Although the order of martensitic transitions reported for other similar B2 shape memory alloys differ from AuZn, the measured $\Delta S_{\text {tr }}$ value for AuZn is well within the range of other $\Delta S_{\text {tr }}$ values. For example, the NiTi alloy exhibits an entropy of $4 \mathrm{~J} \mathrm{~K}^{-1}$ $\mathrm{mol}^{-1}$ [22], and copper based alloys such as $\mathrm{CuZn}$ show about $1 \mathrm{~J} \mathrm{~K}^{-1} \mathrm{~mol}^{-1}$ for their lowtemperature transitions. For AuZn, a Debye temperature of $219 \mathrm{~K}$, obtained from the lowtemperature fit is in favorable agreement with the value of $217 \mathrm{~K}$ for $\mathrm{AgZn}$ [23], although the electronic specific heat for AuZn is nearly half of that reported for AgZn. 


\section{ACKNOWLEDGMENTS}

Tim Darling and Albert Migliori are thanked for the expertise in the RUS measure-

ments. Dan Thoma and F. Chu are thanked for preparing the AuZn single crystals. Jim Smith, Mike Lopez, and Larry Hults are thanked for determining the mechanical properties at liquid helium temperatures.

\section{REFERENCES}

[1] T. Makita, A. Nagasawa, Y. Morii, N. Minakawa, and H. Ohno, Physica B 213-214, 430 (1995).

[2] C. Zener, Phys. Rev. 71846 (1947).

[3] D. A. Porteer and K. E. Easterling, Phase Transformations in Metals and Alloys (Chapman \& Hall, 1992).

[4] A. Migliori and J. Sarrao, Resonant Ultrasound Spectroscopy (Wiley, New York, 1997).

[5] Dynamic Resonance Systems, WY 82435 www.ndtest.com.

[6] W. M. Visscher, A. Migliori, T. M. Bell, and R. A. Reinert, J. Acoustic Soc. Am. 90 2154 (1990).

[7] M. Widn and N. Phillips, Cryogenics 1536 (1975).

[8] J. C. Holste, T. C. Cetas, and C. A. Swenson, Rev. Sci. Instrum. 43670 (1972).

[9] D. L. Martin, Phys. Rev. B. 8, 5357 (1973).

[10] N. E. Phillips, J. P. Emerson, R. A. Fisher, J. E. Gordon, B. F. Woodfield, and D. A. Wright, unpublished data.

[11] D. L. Martin, Rev. Sci. Instrum. 58639 (1987).

[12] B. F. Woodfiled and R. J. Soulen, unpublished data. 
[13] B. F. Woodfield, Ph.D. thesis, University of California-Berkeley (1995).

[14] H. Seitoff, Intermetallics 5, 625 (1997).

[15] T. S. Prevender, and J. F. Smith, J. Appl. Phys. 424680 (1971).

[16] R. D. Field, Private communication.

[17] M. B. Salamon and F. L. Lederman, Phys. Rev. B. 104492 (1974).

[18] B. F. Woodfield, J. Shapiro, R. Stevens, J. Boerio-Goates, R. Putnam, K. Helean, and A. Navratosky, J. Chem. Thermodynamics 31, 1573 (1999).

[19] M. A. Carpenter and E. H. Salje, Eur. J. Mineral. 10693 (1988).

[20] D. Abbe, R. Caudron, and R. Pynn, J. Phys. F. 141117 (1984).

[21] B. Veal and J. Payne, Phys. Rev. B. 551 (1962).

[22] M. P. Punkkinen, K. Kokko, and I. J. Vayrynen, Sol. State Comm. 108567 (1998).

[23] J. P. Abriata, O. J. Bressan, C. A. Luenfo, and D. Thoulouze, Phys. Rev. B. 2, 1464 (1970). 
THE LOW-TEMPERATURE SPECIFIC HEAT AND CRITICAL MAGNETIC FIELD OF $\alpha$-URANIUM SINGLE CRYSTALS

Let us draw up the whole account in terms if easily commensurable.

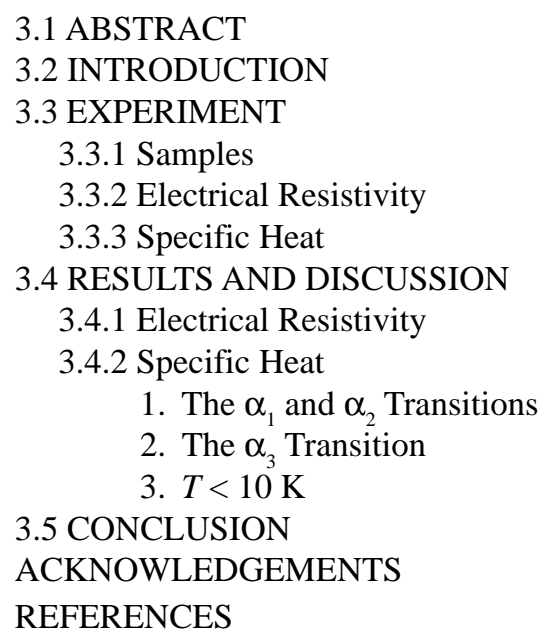

\subsection{ABSTRACT}

The current chapter reports on the specific heat and the electrical resistivity of $\alpha$-uranium at cryogenic temperatures. Measurements were made on new $\alpha$-uranium single crystals that have some unexpected mechanical properties. Despite the fact that $\alpha$-uranium normally work-hardens and often fails in a brittle manner, these crystals bend easily. Presumably, the combination of flexibility and strength comes from twinning in 
response to stress, and these twins can run freely during deformation. Because grain boundaries are not present, we anticipated that the characteristics of the charge density wave (CDW) might be more prominent in these crystals. For these reasons, the specific heat was measured from $T \approx 0.5 \mathrm{~K}$ to $110 \mathrm{~K}$, using semi-adiabatic calorimetry in zero field, and the electrical resistivity was measured from $T \approx 0.1 \mathrm{~K}$ to $0.50 \mathrm{~K}$, in magnetic fields up to $80 \mathrm{mT}$ using a standard four-probe ac technique. An abrupt resistance drop typical of a superconducting transition was observed as the temperature fell below $0.78 \mathrm{~K}$, a temperature at which the resistance fell to $90 \%$ from its original value. A residual resistivity ratio $(\mathrm{RRR}=115)$ was measured from the low-temperature resistivity data. A transition was detected in the specific-heat at $0.65 \mathrm{~K}$, where the specific heat begins to deviate upon cooling from its normal state behavior, also characteristic of a superconducting transition. In addition, three phase transitions were clearly seen in the specific-heat data, located at $T=23 \mathrm{~K}, 36 \mathrm{~K}$, and $42 \mathrm{~K}$. These transitions are consistent with the $\alpha_{3}, \alpha_{2}$, and $\alpha_{1}$ CDW structures that have been previously observed in uranium metal. Analysis of the specific-heat data give an electronic specific heat $(\gamma)=9.13 \mathrm{~mJ} \cdot \mathrm{K}^{-2} \cdot \mathrm{mol}^{-1}$ and a low-temperature limiting Debye temperature $\left(\Theta_{D}\right)=256 \mathrm{~K}( \pm 0.25 \mathrm{~K})$. The highest value obtained previously from calorimetric measurements was $218 \mathrm{~K}$. Our value of $256 \mathrm{~K}$ is in favorable agreement with that previously obtained from elastic constants $250 \mathrm{~K}( \pm 2 \mathrm{~K})$. The appearance of both superconductivity and a CDW state, the agreement between calorimetric and elastic $\Theta_{D}$ values, ductility at room temperature, and a RRR that is three times larger than previously reported values highlight the properties of these new $\alpha$-uranium single crystals. 


\subsection{INTRODUCTION}

Although uranium is a fundamental element to nuclear physics, it exhibits extraordinary solid-state properties [1]. Knowledge of the solid-state properties has benefited from fifty years of metallurgical research aimed at nuclear fuel production. The solidstate properties include a series of three low-temperature charge density wave (CDW) structural phase changes in the normal state and a superconducting transition below $2 \mathrm{~K}$ that shows a large positive isotope effect when the CDW is suppressed by $11 \mathrm{kbar}$ of pressure [2,3]. This superconducting transition in uranium was unique because it was the first example of a metal that becomes a superconductor under pressure without a crystallographic transition [4]. Additionally, superconductivity in uranium raised the important problem of bulk versus filamentary superconductivity for the first time [1]. In the normal state, the first $\mathrm{CDW}$ transition occurs at $43 \mathrm{~K}\left(\alpha_{1}\right)$, the second at $38 \mathrm{~K}\left(\alpha_{2}\right)$, and the last at $22 \mathrm{~K}\left(\alpha_{3}\right)$, where the subscripts 1,2 , and 3 refer to the zero-pressure equilibrium phases. The $\alpha_{1}$ transition is known to be the onset of the CDW state and is associated with a lattice distortion. Below $23 \mathrm{~K}$, all three components of the CDW structure are commensurate with the underlying lattice, while the CDW structure is incommensurate with the underlying lattice between $23 \mathrm{~K}$ and $43 \mathrm{~K}$ [1,5]. There is also a significant increase in the unit cell volume at the $\alpha_{3}$ transition [1].

For the reasons mentioned above, uranium has been the subject of many calorimetric investigations of both polycrystalline and single-crystal samples [6-13]. The resulting electronic specific heat $(\gamma)$ and low-temperature limiting $\Theta_{D}$ parameters obtained from these studies are reported in Table 3.1. After fifty years of multidisciplinary research, one might think that we could simply catalog the engineering properties of uranium and use 
Table 3.1 Values of the electronic specific heat $\gamma$, Debye temperature $\Theta_{D}$, and $T_{c}$ values reported by different investigators.

\begin{tabular}{|c|c|c|c|c|}
\hline Investigators $^{\text {Reference }}$ & $\gamma\left(\mathrm{mJ} \cdot \mathrm{K}^{-2} \cdot \mathrm{mol}^{-1}\right)$ & $\Theta_{D}(\mathbf{K})$ & $T_{c}(\mathrm{~K})$ & Sample \\
\hline Goodman and Schoenberg $1950^{6}$ & 10.6 & 206 & & Polycrystalline \\
\hline Smith and Wolcott $1955^{7}$ & 10.9 & 200 & & Polycrystalline \\
\hline Dempsey, Gordon and Rorner $1963^{8}$ & 12.1 & & & Polycrystalline \\
\hline \multirow[t]{2}{*}{ Gordon et al. $1966^{9}$} & 10.12 & 183 & & \multirow[t]{2}{*}{ Polycrystalline } \\
\hline & 10.03 & 207 & & \\
\hline Flotow and Osborne $1966^{10}$ & 9.88 & 218 & & Polycrystalline \\
\hline \multirow[t]{2}{*}{ Ho et al. $1966^{4}$} & $10.3^{\mathrm{a}}$ & & $<0.1$ & Pseudo-Crystal $^{\mathrm{c}}$ \\
\hline & $12.2^{\mathrm{b}}$ & & 2.0 & Polycrystalline \\
\hline \multirow{4}{*}{$\begin{array}{l}\text { Fisher and Dever } 1968^{\mathrm{d} 11} \\
\text { Crangle and Temporal } 1973^{12}\end{array}$} & & 250 & & Single Crystal \\
\hline & 10.00 & 195 & & Polycrystalline \\
\hline & 9.46 & 203 & & Large-Grained \\
\hline & 9.14 & 210 & & Pseudo-Crystal \\
\hline \multirow[t]{5}{*}{ Bader, Phillips and Fisher $1975^{13}$} & \multicolumn{2}{|l|}{9.59} & 0.27 & Polycrystalline \\
\hline & \multicolumn{2}{|l|}{9.86} & 0.20 & Polycrystalline \\
\hline & \multicolumn{2}{|l|}{9.82} & 0.27 & Large-Grained \\
\hline & \multicolumn{2}{|l|}{9.9} & 0.27 & Large-Grained \\
\hline & \multicolumn{2}{|l|}{9.14} & $<0.1$ & Single Crystal \\
\hline Current Study 2000 & 9.13 & 256 & 0.65 & Single Crystal \\
\hline \multicolumn{5}{|c|}{ 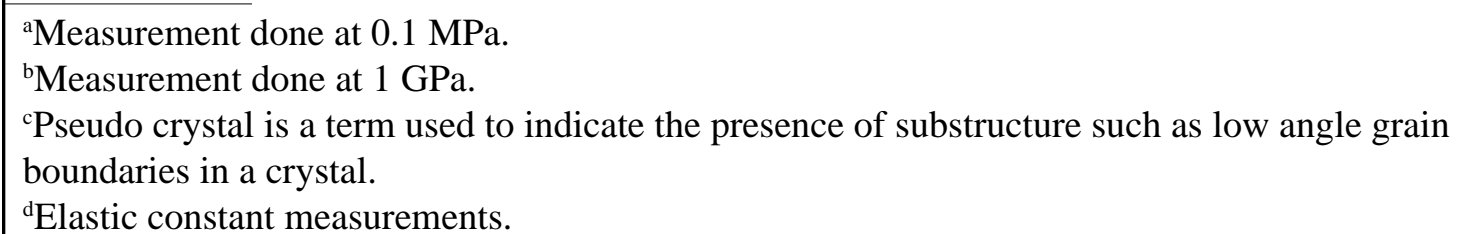 } \\
\hline
\end{tabular}

this to know, for example, how superconductivity and the CDW state coexist. However, years of research have shown that $T_{c}$ and the CDW state depend too sensitively on things such as impurities, temperature, and method of fabrication to be predictable. Accordingly, many ambiguities associated with the experimental situation and differing values summarized in Table 3.1 have been attributed to the purity of samples and the quality of single crystals. For example, calorimetric measurements made at $10 \mathrm{kbar}$ are consistent with 
magnetic measurements and have confirmed the presence of bulk superconductivity with a $T_{c}$ of $2 \mathrm{~K}$ [14]; however, calorimetric measurements at zero pressure give widely different values for $T_{c}$ depending on the sample. One model to explain this variation has been that certain impurities and/or filaments of strained material can help stabilize a bulk superconductor by suppression of the CDW [15]. In particular, it has been reported that a $T_{c}$ between $0.25 \mathrm{~K}$ and $0.80 \mathrm{~K}$ is due to strain filaments within the metal [16]. The values of the electronic specific heat and the Debye temperatures in the low-temperature limit appear to be influenced by the substructure within a crystal. The differences in the Debye temperatures have been ascribed to variations in the CDW suppression [1]. As noted in Table 3.1, the values prior to the current study determined from $C / T$ vs. $T^{2}$ plots between 0 and $4 \mathrm{~K}$, vary from $183 \mathrm{~K}$ to $218 \mathrm{~K}$. One can see that these values tend to be lower for polycrystalline samples. In contrast, the Debye temperature calculated from the singlecrystal elastic constants is $250 \mathrm{~K}$. The large difference between this value and the thermal values is rare for a pure element.

Recently, we prepared new single crystals of $\alpha$-uranium metal using electrochemical techniques. Although orthorhombic $\alpha$-uranium normally work-hardens rapidly and often fails in a brittle manner, these crystals bend easily and do not work-harden. They were grown by electro-transport through a molten salt bath formed as dendrites [17]. These samples differ from samples used in previous investigations in that they are relatively strain free and show facets. It was anticipated that the characteristics of the low-temperature properties of uranium might be more prominent in these crystals because defects that typically pin the CDW state are not present. Although $\alpha$-uranium has been the subject of numerous resistivity and calorimetric investigations, we were motivated to revisit the 
low-temperature physics of uranium with these new high quality single crystals. The electrical resistance measurements reported here were undertaken to clarify the nature of superconductivity in single crystal $\alpha$-uranium at zero pressure and to measure the critical magnetic field. The specific-heat measurements were undertaken to search for superconductivity at zero pressure and to obtain the thermodynamic properties of the CDW state. In addition, we investigate the $16 \%$ difference between the Debye temperatures previously obtained from specific heat data and those calculated from single-crystal elasticity measurements.

\subsection{EXPERIMENT}

\subsubsection{Samples}

The uranium crystals were grown by electro-transport through a molten salt bath of $\mathrm{LiCl}-\mathrm{KCl}$ eutectic containing on the order of $3 \mathrm{wt} . \% \mathrm{UCl}_{3}[18]$. The uranium was deposited onto a stainless steel cathode as dendrites in the form of parallelogram-edged platelets, often diamond shaped. These individual platelets within the dendrites are single crystals of $\alpha$-uranium. Because the uranium was deposited at temperatures below the $\alpha-\beta$ transformation temperature, they are strain free and nearly perfect. The crystals were characterized by a back-reflection Laue technique that determined that the $c$-axis was orthogonal to the plate. Parallelepiped strips and short cylindrical samples were shaped by spark-erosion cutting, and were cleaned in concentrated $\mathrm{HNO}_{3}$ and electropolished in $\mathrm{H}_{3} \mathrm{PO}_{4}$ 


\subsubsection{Electrical Resistivity}

The electrical resistivity was measured using a standard four-probe ac technique. The magnetic field and current were parallel and perpendicular to the $c$-axis, respectively. A Linear Research LR-700 resistance bridge, which implemented a standard four-terminal ac measurement at $16 \mathrm{~Hz}$, was used in this measurement. The small resistance of the crystal (about $12 \mu \Omega$ ) below $2 \mathrm{~K}$ required a large excitation current of $1.5 \mathrm{~mA}$. The electrical resistance above $2 \mathrm{~K}$ was measured using a standard four-terminal ac technique with an excitation current of $3 \mathrm{~mA}$. The resistivity was calculated from the dimension of the sample with an estimated absolute uncertainty of $15 \%$.

\subsubsection{Specific Heat}

The specific heat was measured in zero-magnetic field using an apparatus designed

around a ${ }^{3} \mathrm{He}$ insert capable of attaining temperatures as low as $0.4 \mathrm{~K}$. Two single crystals of uranium, weighing a total of $0.5 \mathrm{~g}$, were stacked and thermally attached to the sample platform with a thin layer of Apiezon $\mathrm{N}$ grease. The specific heat of the empty sample platform was measured separately, and the specific heat of the empty sample platform and the Apiezon $\mathrm{N}$ grease was subtracted from the total specific heat to obtain the specific heat of the sample. A semi-adiabatic pulse technique was used to measure the specific heat from the lowest temperature up to $10 \mathrm{~K}$, and this technique was also used to map the $\alpha_{3}$ transition. Semi-adiabatic conditions are obtained by adding enough heat to obtain a flat drift at each temperature. An isothermal technique was used to measure the specific heat from $10 \mathrm{~K}$ to $110 \mathrm{~K}$. In the isothermal technique, a constant temperature block is heated to a series of predetermined temperatures and the sample is allowed to drift toward the block temperature before and after a heating pulse. The details of the 
semi-adiabatic pulse and isothermal techniques and thermometry are described elsewhere [19-20].

\subsection{RESULTS AND DISCUSSION}

\subsubsection{Electrical Resistivity}

We observed an abrupt drop in the resistance of the crystal as the temperature fell below about $0.78 \mathrm{~K}$, the temperature at which the resistance appears to reach its minimum value. The quantities are graphically defined in Figure 3.1. The critical temperature, $T_{c}$, is taken to be the temperature at which the resistance falls to $90 \%$ of its value in the normal state. The dependence of $T_{c}$ on $H$ is shown in Figure 3.2. These data are taken to mark the superconducting critical magnetic field, $H_{c}$. The broad resistive transition, which is smoothed but is otherwise uncorrected for what we believe to be a small instru-

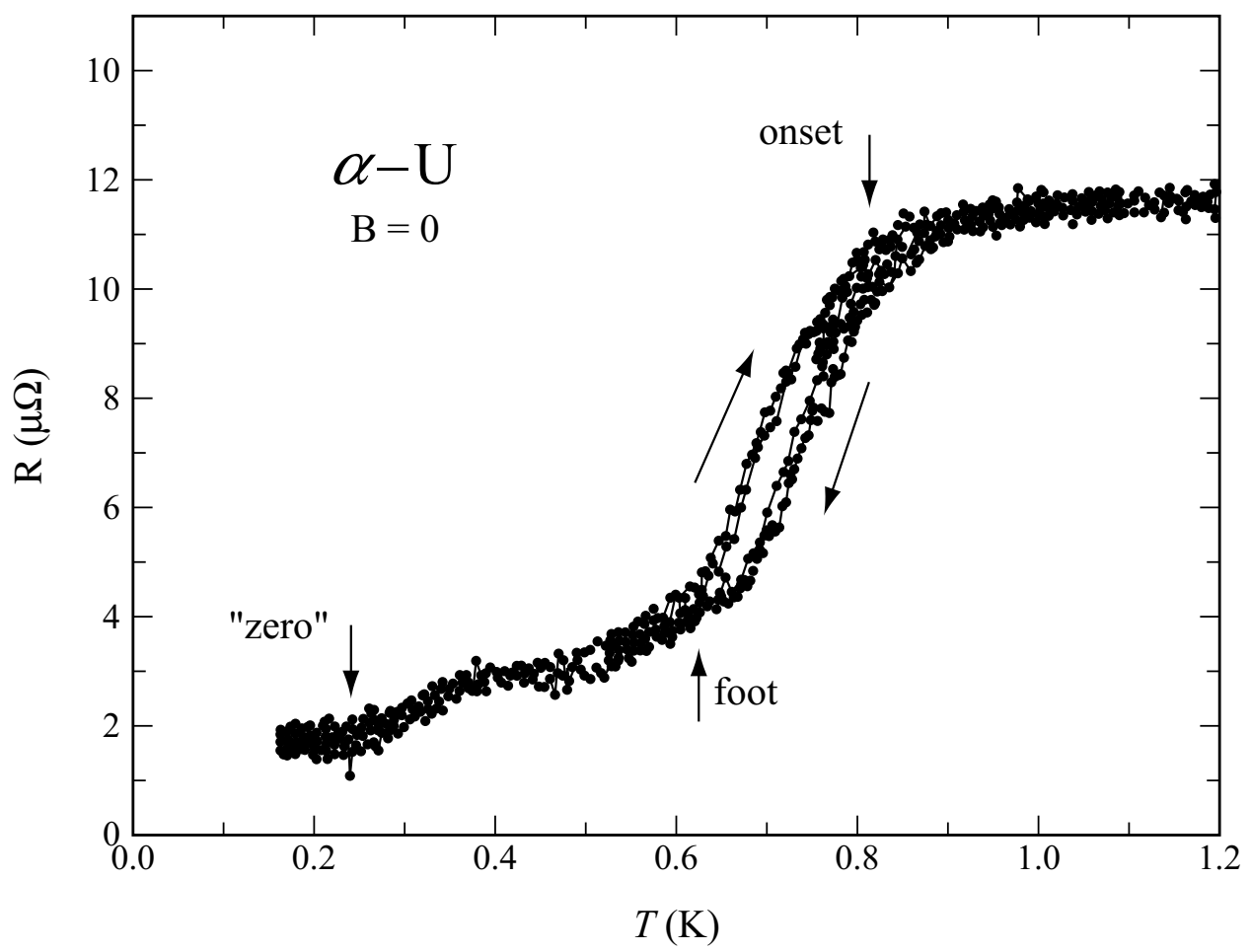

Figure 3.1. Graphical definition of terms used in describing the resistivity data. We base $T_{c}$ on the foot value. 


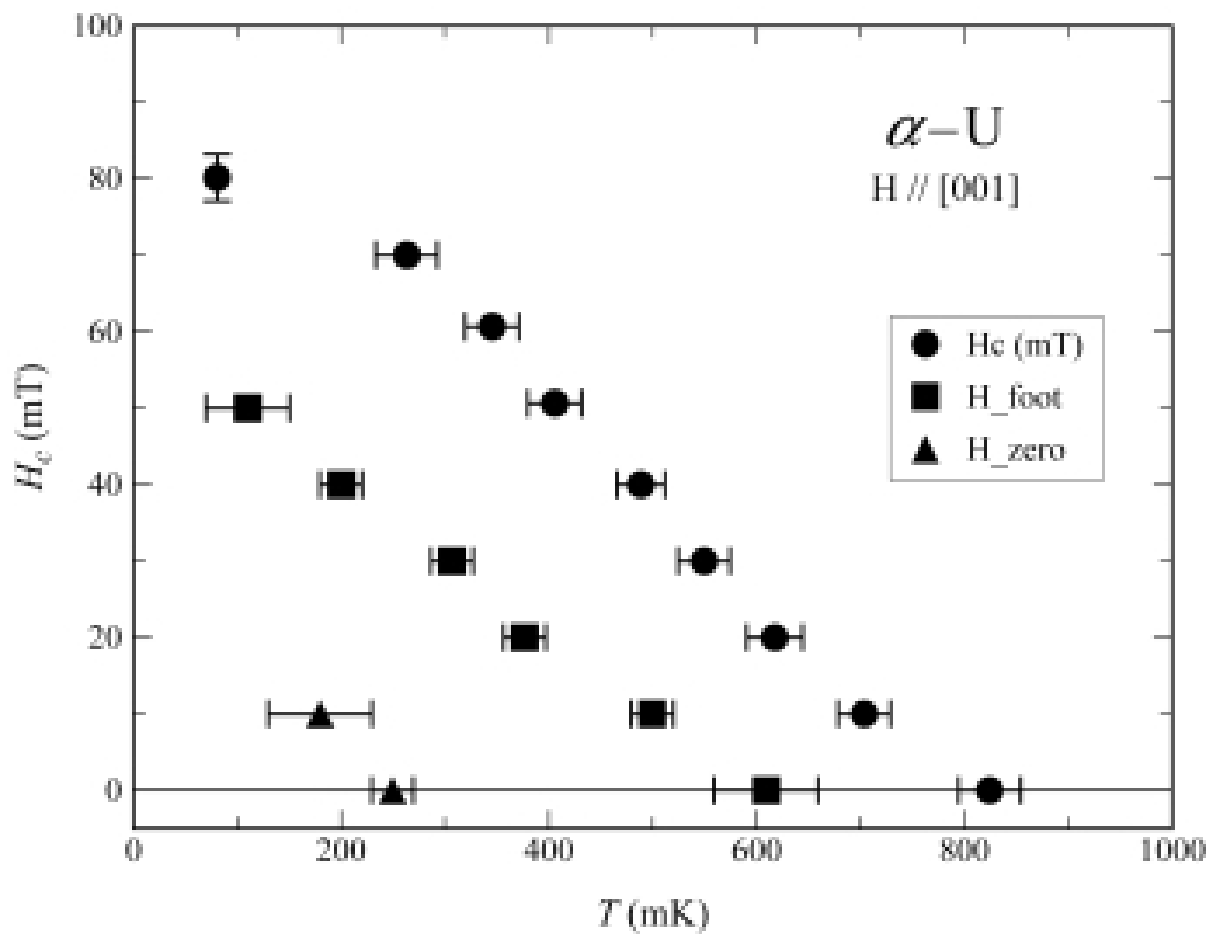

Figure 3.2. A graph of $H_{c}(T)$ versus $T$. The magnetic field was parallel to the [001] direction in this experiment.

mental dc offset, is shown in the inset of Figure 3.3. One can see a slight positive curvature just below $T_{c}$ in the $H_{c}(T)$ data, which evolves into a quadratic temperature dependence as the temperature approaches $0 \mathrm{~K}$. However, a fit of this data to Equation (3.1) gives the qualitative form expected for Type I superconductors

$$
H_{c}(T)=H_{c}(0)\left[1-\left(T / T_{c}\right)^{2}\right]
$$

with $H_{c}(0)=74 \pm 4 \mathrm{mT}$ and $T_{c}=0.78 \pm 0.05 \mathrm{~K}$, shown as the solid line in Figure 3.3, and characterizes the data reasonably well.

The temperature dependence of the resistivity between $2 \mathrm{~K}$ and $50 \mathrm{~K}$ is shown in Figure 3.4. Diagonal arrows denote the direction in which the temperature was changing during data acquisition. Vertical arrows denote the position of features we associate with the $\mathrm{CDW}$ transitions at $\alpha_{1}=42 \mathrm{~K}, \alpha_{2}=37 \mathrm{~K}$, and $\alpha_{3}=23 \mathrm{~K}$ in good agreement with the 


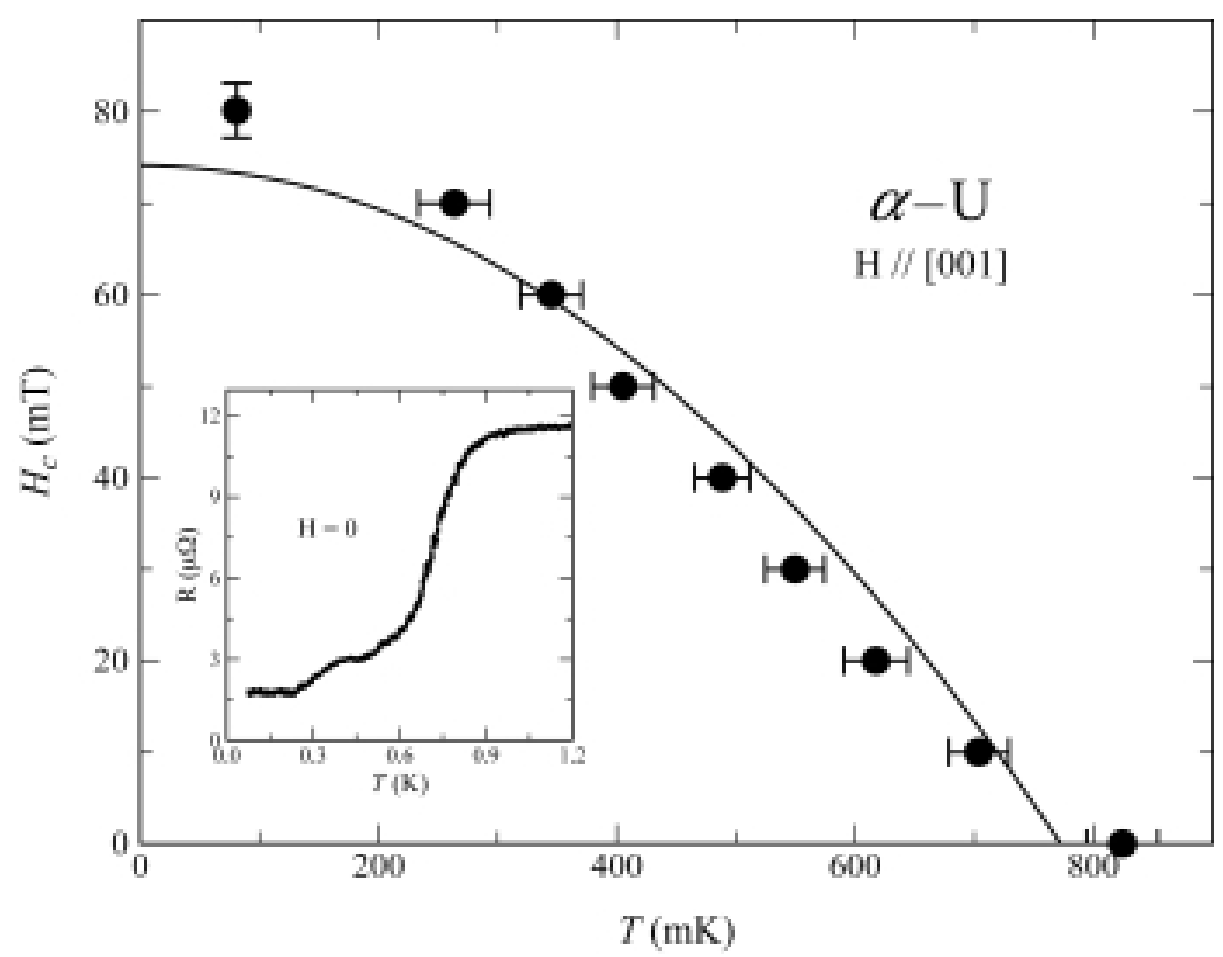

Figure 3.3. A graph of $H_{c}(T)$ versus $T$. The $H_{c}(T)$ data evolves into quadratic temperature dependence in the low-temperature limit. Inset: The broad resistive transition arises from what we believe to be a small instrumental dc offset.

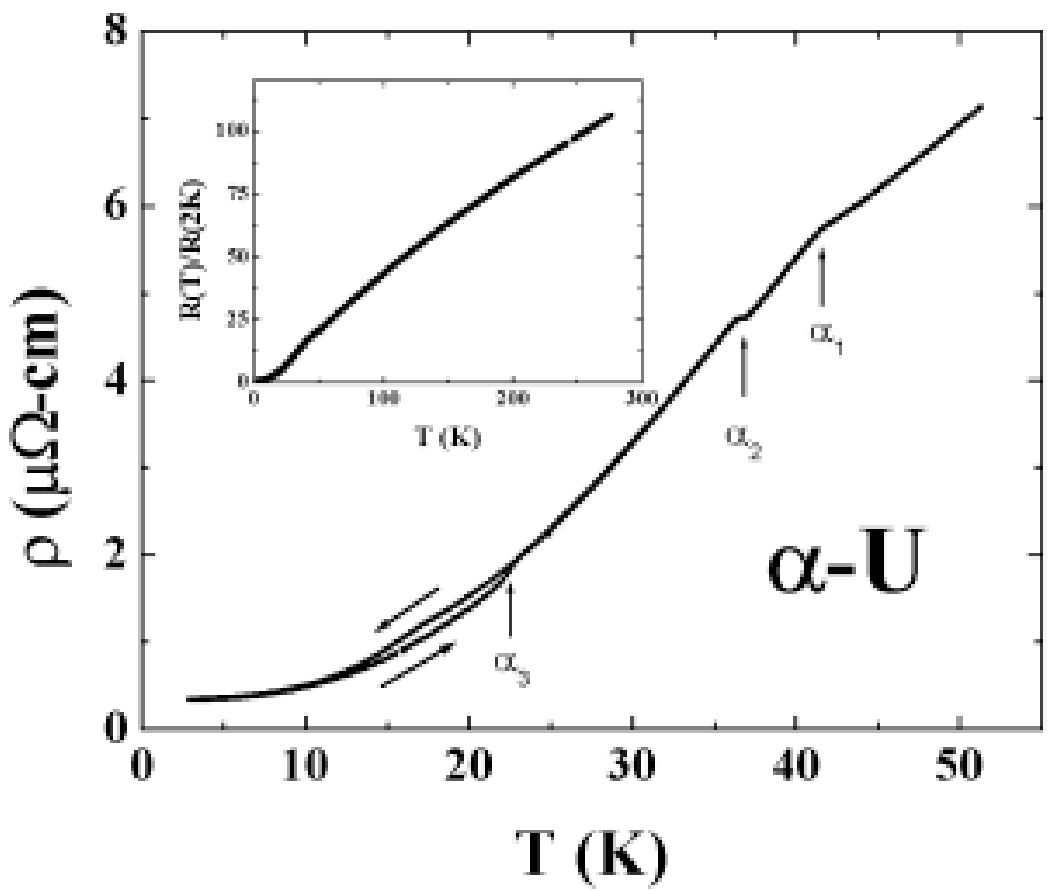

Figure 3.4. The temperature dependence of the resistivity between $T \approx 2 \mathrm{~K}$ and $50 \mathrm{~K}$. Diagonal arrows denote the direction in which the temperature was changing during data acquisition. Vertical arrows denote the position of the CDW features. Inset: The resistivity up to room temperature divided by its value near $2 \mathrm{~K}$. 
values in the literature [1]. The inset shows the resistivity up to room temperature divided by its value near $2 \mathrm{~K}$. Though similar in shape to earlier measurements reported in the literature [1], our resistivity data is qualitatively different in that the features associated with all three CDW transitions are clearly visible: a break in slope at $\alpha_{1}$, a step-like feature at $\alpha_{2}$ (at which a small thermal hysteresis appears), and an abrupt onset of significant thermal hysteresis at $\alpha_{3}$. The residual resistivity ratio (RRR) is about 115 which is three times higher than any RRR reported previously [1]. The magnetoresistance (with $H$ parallel to the crystalline $c$-axis) is very large and featureless, reaching approximately $750 \%$ at $18 \mathrm{~T}$ and at $2 \mathrm{~K}$, a result which is also consistent with high-purity samples. A full description of our measurements in high magnetic fields will be reported elsewhere.

\subsubsection{Specific Heat}

The specific heat of uranium, with four low-temperature anomalies, is shown in Figure 3.5. As uranium cools to approximately $41 \mathrm{~K}$, the $\alpha_{1}$ appears as a round hump in the specific heat data. In contrast, the $\alpha_{2}$ transition at approximately $38 \mathrm{~K}$ appears as a sharp peak. Further cooling of uranium to approximately $23 \mathrm{~K}$ results in yet another broad transition, the $a_{3}$ transition. Shown in the inset of Figure 3.5 is the specific heat

data below $4 \mathrm{~K}$. Starting at $2 \mathrm{~K}$ there is a gradual upturn, typical of a ${ }^{235} \mathrm{U}$ hyperfine interaction, and there is a steep upturn at $0.65 \mathrm{~K}$ typical of superconductivity. In order to calculate the excess specific heat from 10 to $110 \mathrm{~K}$, the lattice specific heat was estimated by fitting the data using a hand spline. 


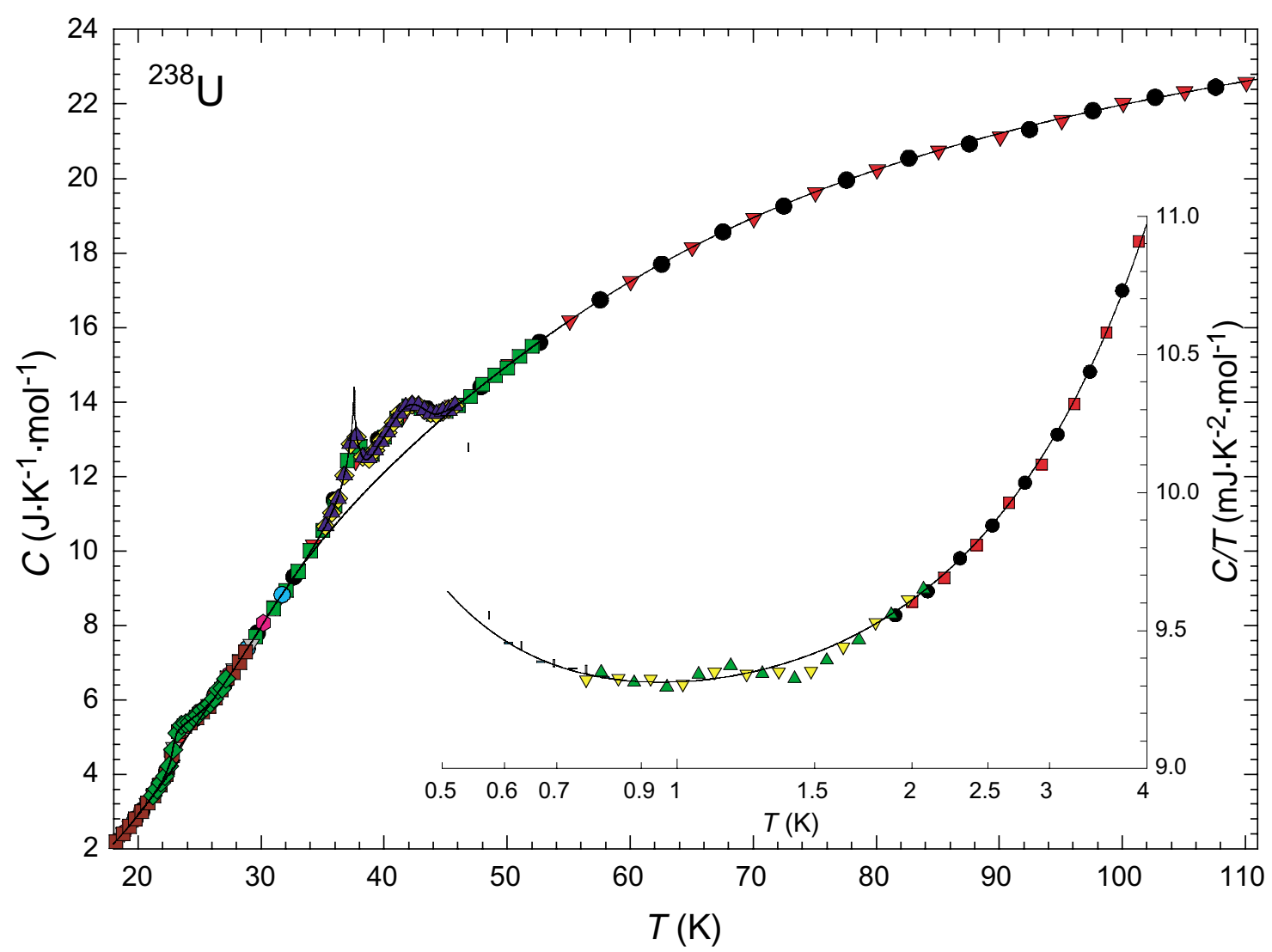

Figure 3.5. The specific heat of $\alpha$-U as a function of temperature over a wide temperature interval. The $\alpha_{1}, \alpha_{2}$, and $\alpha_{3}$ transitions are easily observable. Inset: The details of the specific heat below $4 \mathrm{~K}$. The broad upturn is associated with ${ }^{235} \mathrm{U}$ hyperfine interaction and the sharp upturn is consistent with the onset to superconductivity.

\section{The $\alpha_{1}$ and $\alpha_{2}$ Transitions}

Shown in Figure 3.6 are the $\alpha_{1}$ and $\alpha_{2}$ transitions where a lattice estimation has been subtracted in order to more clearly show the excess specific heat of the transitions.

In order to map out the transition, several sets of measurements were made through this region each with successively smaller $\Delta T$ intervals; the different data symbols in Figure 3.6 reflect different passes through the region. Different cooling rates through the $\alpha_{1}$ and $\alpha_{2}$ transitions were also used but with no apparent effect on the data. The fit of each transition is extrapolated to the baseline to permit integration of each individual transition. In particular, $\alpha_{2}$, which is clearly a symmetrical transition, was extrapolated to the 


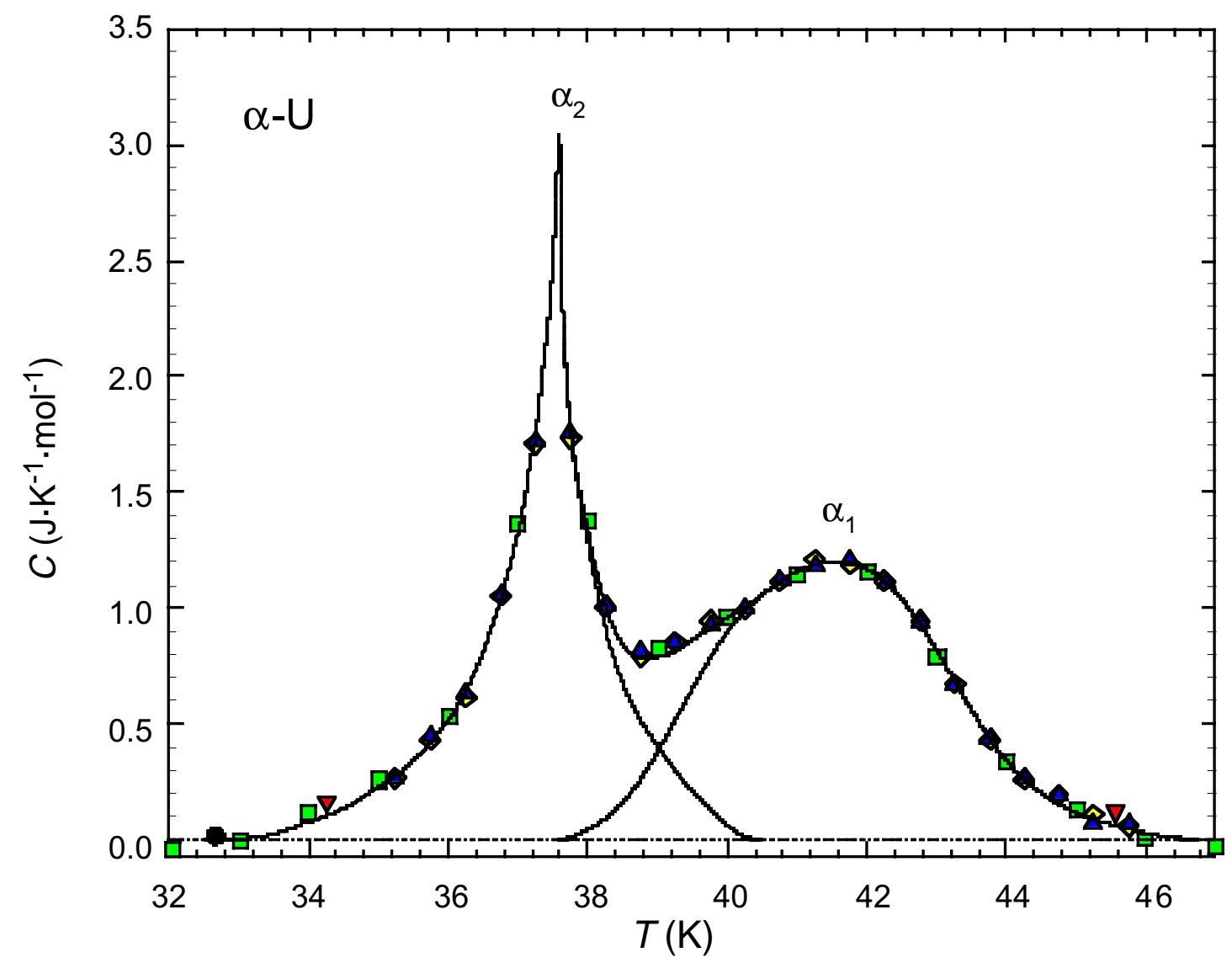

Figure 3.6. The excess specific heat of the $\alpha_{1}$ and $\alpha_{2} \mathrm{CDW}$ transitions. The lattice specific heat has been subtracted from the total specific heat in order to more clearly show the shape of these transitions.

high temperature side (maintaining the symmetrical character of the transition) and was then subtracted from the full transition (the lattice having already been subtracted) to obtain an estimate of the $\alpha_{1}$ transition. We tested the self-consistency of our extrapolations by adding the two individual transitions, which resulted in the fit through the full transition as shown in Figure 3.6. Integration of the $C / T$ data gives an entropy of transition that is the same, within experimental error, for each phase change $\Delta S_{\alpha 1}=0.12 \pm 0.01 \mathrm{~J} \cdot \mathrm{K}^{-1} \cdot \mathrm{mol}^{-1}$ and $\Delta S_{\alpha 2}=0.11 \pm 0.01 \mathrm{~J} \cdot \mathrm{K}^{-1} \cdot \mathrm{mol}^{-1}$

The transition at $41 \mathrm{~K}\left(\alpha_{1}\right)$ involves both structural and electronic changes [1]. Changes in the lattice parameter are observed near $40 \mathrm{~K}$ [1] and the electrical resistivity 
changes also at $41 \mathrm{~K}$. The shape of the heat capacity peak associated with this transition is somewhat unusual and is clearly not that expected of a strongly first-order transition. The shape of the heat capacity peak, coupled with the lack of significant thermal hysteresis in either the heat capacity measurements or the resistivity measurements, rule out a strong first-order character for this transition.

In contrast, the transition at $37 \mathrm{~K}\left(\alpha_{2}\right)$ seems to be associated solely with electronic changes since features are observed only in the electrical resistivity and not in the neutron scattering measurements [1] of lattice parameters at this temperature. The heat capacity anomaly differs in shape from that in the $\alpha_{1}$ region where the $\alpha_{2}$ peak is much sharper and reaches a higher heat capacity than that for the $\alpha_{1}$ peak. The sharp character of the $\alpha_{2}$ transition and the presence of a transition in the resistivity data at the same temperature is consistent with a first-order change in the electronic state. This observation is supported by recent first principles total energy calculations on the CDW state the results of which suggest that the $\alpha_{2}$ transition is a modified $\alpha_{1} \mathrm{CDW}$ state caused by directional nesting of the Fermi surface [21].

\section{The $\alpha_{3}$ Transition}

From $18 \mathrm{~K}$ to $26 \mathrm{~K}$ an estimate of the lattice specific heat was interpolated through the $\alpha_{3}$ transition at $23 \mathrm{~K}$ and is shown in Figure 3.7. A baseline could not be drawn from the low-temperature to the high-temperature side of the transition without a discontinuous change. This obvious step in the baseline is consistent with a significant lattice coupling associated with the $\alpha_{3}$ transition. To confirm this result, the specific heat was measured after cooling quickly through the transition, and the resulting specific-heat data show an almost completely quenched transition. However, for slow cooling, the transition appears 


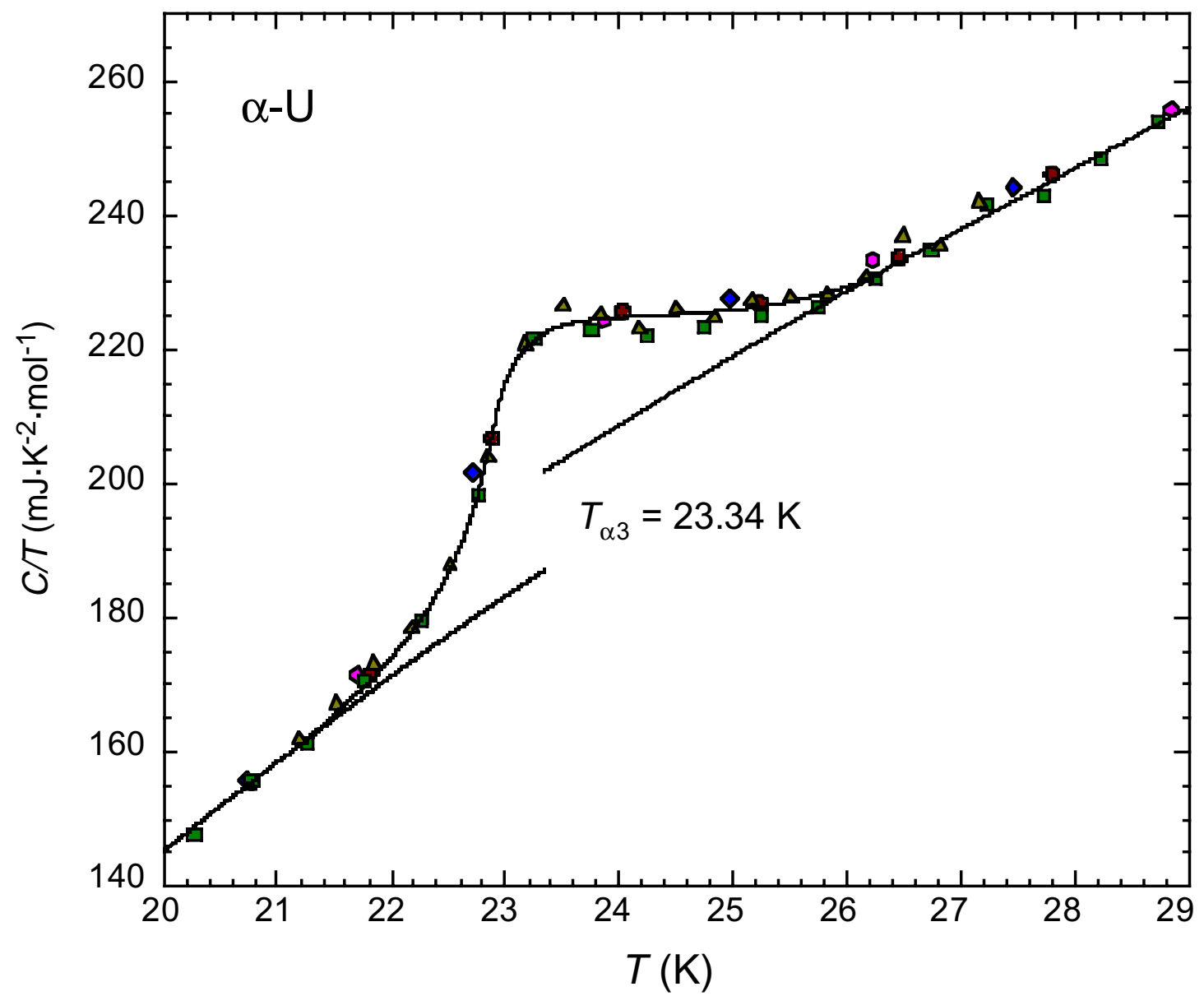

Figure 3.7. The $\alpha_{3}$ CDW transition is shown on an expanded scale with lines representing the baseline extrapolations. The obvious baseline offset in the low- and high-temperature side of the transition is indicative of a first-order lattice transition.

as a rise in the specific heat with a rounded maximum. The presence of the step-change in the baseline is strong evidence for a first-order phase change. Figure 3.8 illustrates the shape of the transition and the discontinuous baseline in the excess specific heat. The abrupt drop in the transition, as seen in Figure 3.8, arises from the baseline offset, taken to be at the transition temperature. Integration of the $C / T$ data yields an entropy of transition $\Delta S_{a 3}=0.05 \pm 0.01 \mathrm{~J} \cdot \mathrm{K}^{-1} \cdot \mathrm{mol}^{-1}$, an entropy that is half of the two higher temperature transitions. 


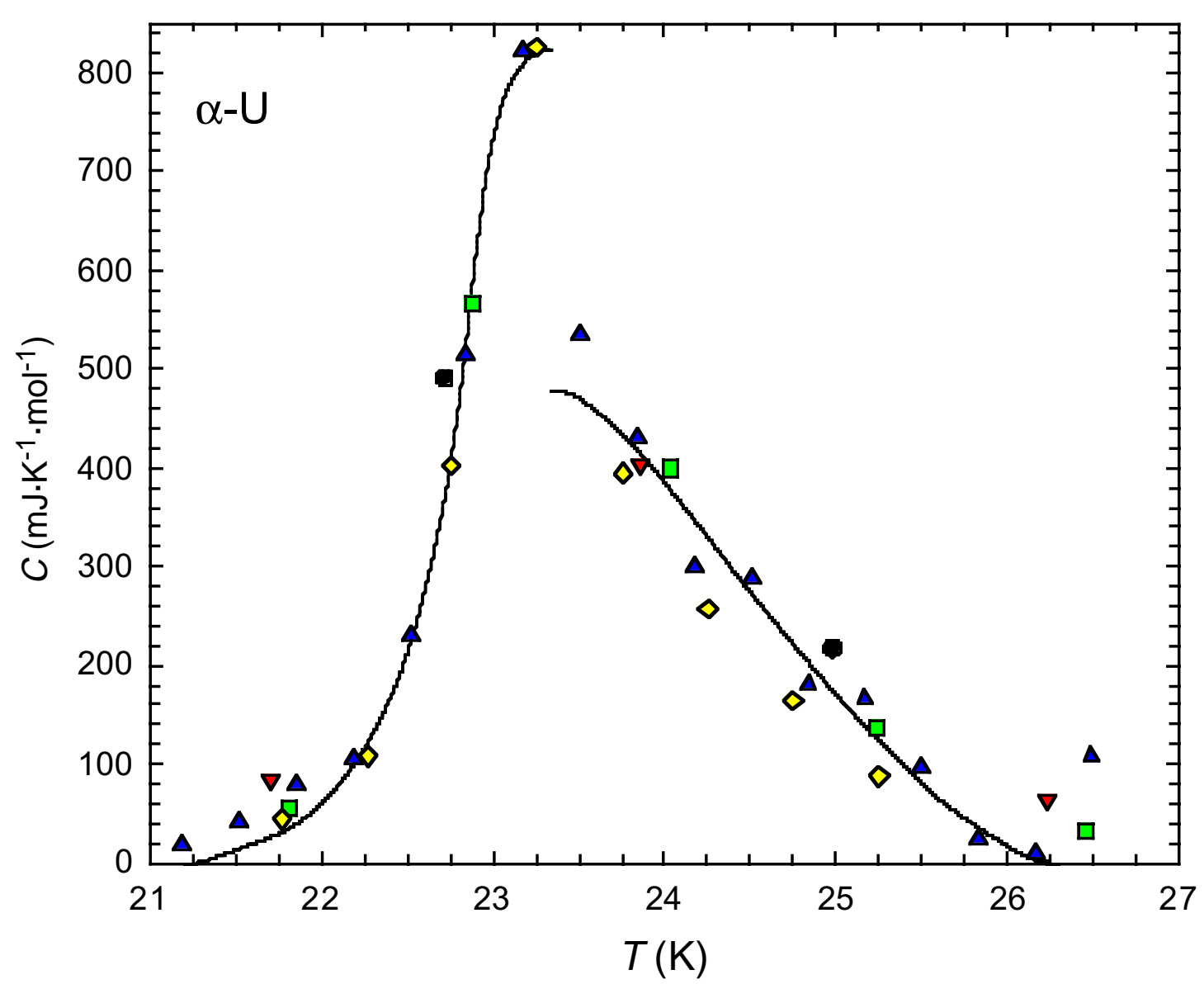

Figure 3.8. The excess specific heat of the $\alpha_{3} \mathrm{CDW}$ transition with the baseline subtracted. The abrupt drop in the transition is caused by the baseline offset.

\section{3. $T<10 \mathrm{~K}$}

The heat liberated from the depleted ${ }^{238} \mathrm{U}$ sample during its $\alpha$-decay process prevented cooling to temperatures below $0.5 \mathrm{~K}$ in the specific-heat measurements. The electronic term $(\gamma)$ and the Debye temperature $\left(\Theta_{\mathrm{D}}\right)$ were obtained from a fit from $1.2 \mathrm{~K}$ to $4 \mathrm{~K}$ using a conventional $C / T$ versus $T^{2}$ extrapolation represented by Equation (3.2), and is shown in Figure 3.9

$$
C / T=\gamma+B_{3} T^{2}
$$

A Debye temperature of $256 \mathrm{~K}( \pm 0.25 \mathrm{~K})$ and an electronic specific heat of $9.13 \mathrm{~mJ} \cdot \mathrm{K}^{-2} \cdot \mathrm{mol}^{-1}$ was also obtained from this fit. The lattice specific heat was fitted over 


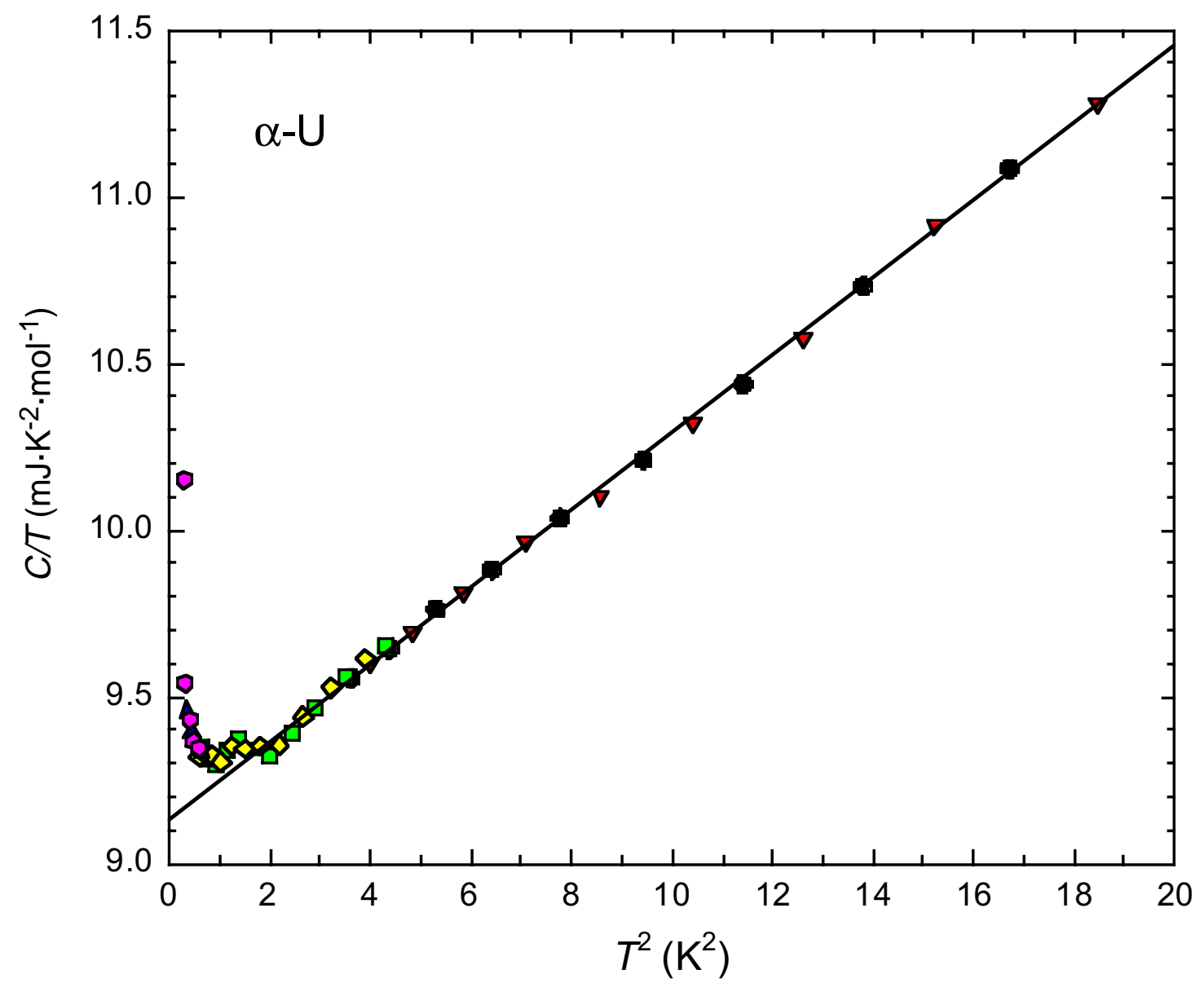

Figure 3.9. A graphical representation of the fit of the low-temperature specific heat data to Equation (3.2) from which $\gamma$ and $\Theta_{D}$ were determined.

a more extended temperature range from 1.2 to $10 \mathrm{~K}$ using the low-temperature expansion of the Debye function represented by Equation (3.3)

$$
C_{V}=\gamma T+B_{3} T^{3}+B_{5} T^{5}+B_{7} T^{7}+B_{9} T^{9}+B_{11} T^{11}+B_{13} T^{13},
$$

where the $\gamma$ and $B_{3}$ terms were fixed at the values obtained from the $C / T$ extrapolation, and the required number of adjustable parameters for the lattice $\left(B_{n}\right)$ increased until a suitable fit was obtained. It is interesting to note that a $B_{13}$ term is required to fit uranium up to $10 \mathrm{~K}$. By way of comparison, only a $B_{9}$ term is needed to represent the copper lattice up to approximately $30 \mathrm{~K}$ [17]. 
One can see a gradual upturn in the low-temperature $C$ vs. $T$ data as shown in the inset of Figure 3.5. This upturn is the nuclear specific heat and arises from the ${ }^{235} \mathrm{U}$ hyperfine interaction. This hyperfine interaction manifests itself as a nuclear Schottky anomaly which has a $T^{-2}$ temperature dependence in the high-temperature limit. The nuclear specific heat was fitted to the expression in Equation (3.4)

$$
C=\gamma T+B_{3} T^{3}+A T^{-2}
$$

where $\gamma$ and $B_{3}$ were fixed at the values obtained from the fit to Equation (3.2) and is shown as the line through the data in the inset of Figure 3.5. ${ }^{235} \mathrm{U}$ concentration of 0.58 at. $\%$ was estimated for our sample using the $A$ term obtained from the fit and correlations of the hyperfine term to ${ }^{235} \mathrm{U}$ concentrations reported previously in the literature [4]. The ${ }^{235} \mathrm{U}$ hyperfine contribution, $C_{\text {hyp }}$, was subtracted from the specificheat data and the resulting fit to the data is shown in Figure 3.10. There is an obvious upturn in the $C / T$ vs. $T$ data at approximately $0.65 \mathrm{~K}$, consistent with the superconducting transition detected in the resistivity data. The origin of the small oscillation in our specific heat data near 1.2 K remains unknown; however based on previous measurements, this oscillation it is not an artifact of our temperature scale [19].

\subsection{CONCLUSION}

We present experimental evidence indicating that $\alpha$-uranium is likely to be a bulk superconductor at zero pressure, although additional specific-heat measurements in a magnetic field at lower temperatures are needed to completely resolve this issue. An abrupt drop in the resistivity was observed at $0.78 \mathrm{~K}( \pm 0.05 \mathrm{~K})$ and a critical field, $H_{c}(0)=74 \mathrm{mT}( \pm 4 \mathrm{mT})$ was obtained from the low-temperature electrical resistivity 


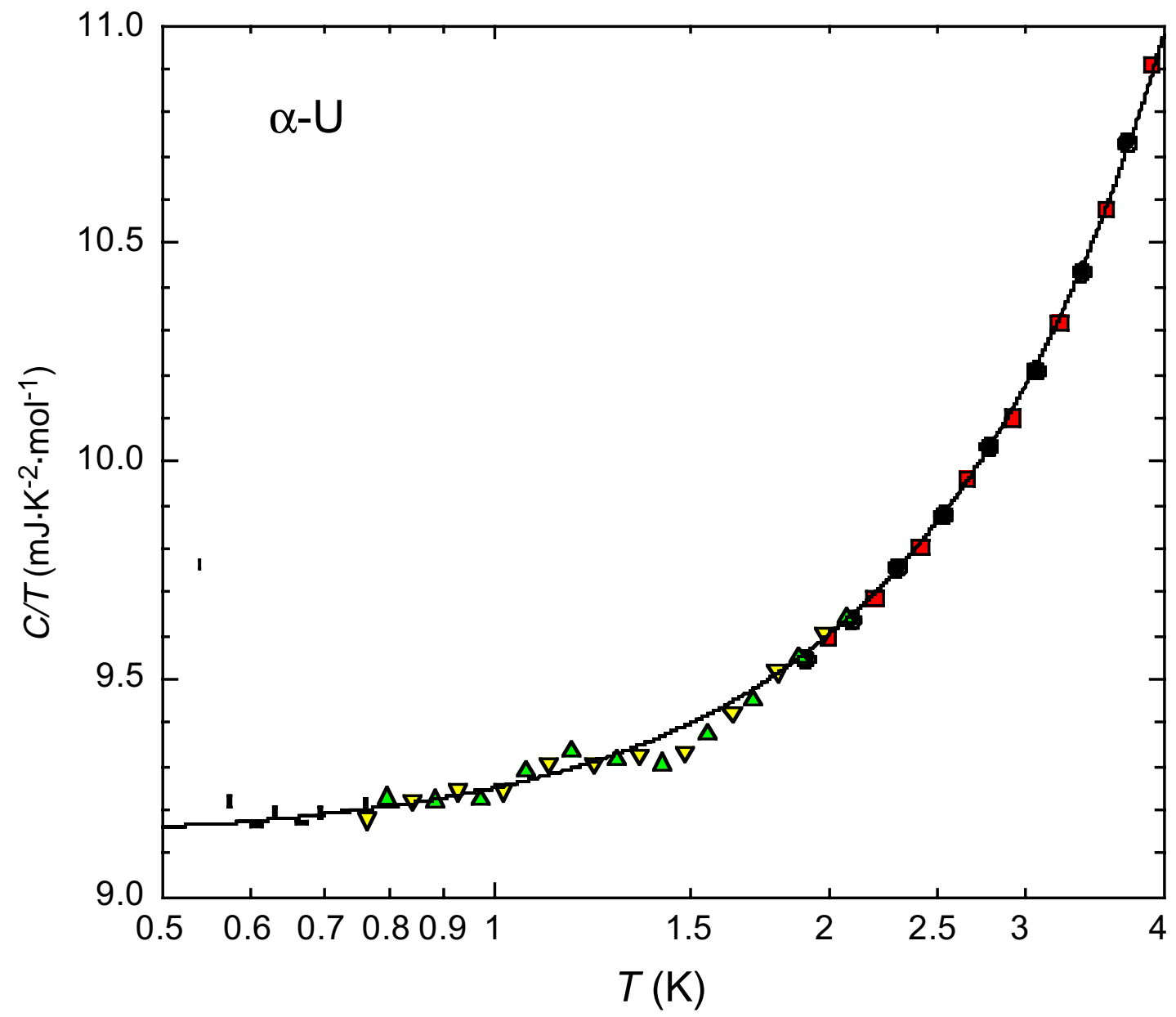

Figure 3.10. The low-temperature specific heat with the nuclear with the nuclear hyperfine contribution subtracted. The origin of the oscillation in the specific heat at $1.2 \mathrm{~K}$ is not known, although it is not an artifact of our temperature scale.

measurements. A steep upturn in the normal-state specific heat was observed at $0.65 \mathrm{~K}$ $( \pm 0.05 \mathrm{~K})$, a temperature that is considerably higher than the upper-limit $T_{c}$ value of $0.1 \mathrm{~K}$ reported by Phillips et. al. [13] for another high-quality uranium sample. The resistivity data for the superconducting transition fits the qualitative form expected for a Type I superconductor. Our electronic specific heat value $\left(\gamma=9.13 \mathrm{~mJ} \cdot \mathrm{K}^{-2} \cdot \mathrm{mol}^{-1}\right)$ is within experimental error with the value previously reported by Phillips et al. [13] and Crangle and Temporal [12]. In contrast, our $\Theta_{D}$ value of $256 \mathrm{~K}$ is higher than previously reported 
calorimetric values and agrees more closely with the value obtained from elastic constant measurements $(250 \mathrm{~K})[11]$. Agreement between $\Theta_{D}$ values and from elastic constants and the thermal value indicate that the lattice appears to be strain free, a result that is substantially different than those obtained on single crystals grown by strain-anneal and perhaps explain the higher $T_{c}$. This result is confirmed by the measured $\mathrm{RRR}=115$ for these crystals.

The characteristics of the CDW appear to be more prominent in this sample as we observe transitions $1 \mathrm{~K}$ higher than previously measured. The $\alpha_{1}$ transition has an unusual shape and is not likely to have strong first-order character. The transition at $37 \mathrm{~K}$ shows first-order character and has a sharp peak in the excess specific heat. It is noteworthy that the entropies of the $\alpha_{1}$ and $\alpha_{2}$ transitions are equal, within experimental error. We also show evidence that the $\alpha_{3}$ transition is first order and has a significant lattice component. The entropy of the $\alpha_{3}$ transition is half that of the $\alpha_{1}$ and $\alpha_{2}$ transitions.

Uranium is in a privileged position because it is an element that offers a unique opportunity to study how superconductivity and the CDW coexist. It is noteworthy to mention that recent work on quantum criticality [22] is beginning to explain how the two can coexist.

\section{ACKNOWLEDGMENTS}

We are grateful to Al Arko for review of this manuscript. We are indebted to Norman Phillips and Robert Fisher who did much of the pioneering work on the heat capacity of uranium. Part of this work was carried out at Brigham Young University and also at the Los Alamos National Laboratory supported under the Primary Physics Experiments 
Program, under the auspices of the Department of Energy. This work was partially supported by the National Science Foundation (NSF) under DMR-0071947. The critical field and resistivity measurements were made at the National High Magnetic Field Laboratory, which is supported by NSF Cooperative Agreement No. DMR-9527035, and by the state of Florida.

\section{REFERENCES}

[1] G. H. Lander, E. S. Fisher, and S. D. Bader, Adv. Phys. 43, p. 1 (1994).

[2] E. S. Fisher and H. J. McSkimin, Phys. Rev. 124, p. 67 (1961).

[3] R. D. Fowler, B. T. Matthias, L. P. Asprey, H. H. Hill, J. D. Lindsay, C. E. Olsen, and R. W. White, Phys. Rev. Lett. 19, p. 892 (1967).

[4] J. C. Ho, N. E. Phillips, and T. F. Smith, Phys. Rev. Lett. 17, p. 694 (1966).

[5] H. G. Smith, N. Wakacayashi, W. P. Crummett, R. M. Nicklow, G. H. Lander, and E. S. Fisher, Phys. Rev. Lett. 44, p. 1612 (1980).

[6] B. B. Goodman and D. Schoenberg, Nature 165, p. 441 (1950).

[7] P. L. Smith and N. M. Wolcott, Supplement au Bulletin l'Institut du Friod (Paris) 1, p. 283 (1955).

[8] C. W. Dempsey, J. E. Gordon and R. H. Romer, Phys. Rev. Lett. 11, p. 547 (1963).

[9] J. E. Gordon, H. Montgomery, R. J. Noer, S. Pickett, and R. Tobon, Phys. Rev. 152, p. 432 (1966).

[10] H. E. Flotow and D. W. Osborne, Phys. Rev. 151, p. 564 (1966).

[11] E. S. Fisher and D. Dever, Phys. Rev. 179, p. 607 (1968).

[12] J. Crangle and J. Temporal, J. Phys. F. 3, p. 1097 (1973). 
[13] S. D. Bader, N. E. Phillips, and E. S. Fisher, Phys. Rev. B. 12, p. 4929 (1975).

[14] C. W. Dempsey, J. E. Gordon, and R. H. Romer, Phys. Rev. Lett. 11, p. 547 (1963).

[15] W. E. Gardner and T. F. Smith, Phys. Rev. 154, p. 309 (1967).

[16] E. S. Fisher, T. H. Geballe, and J. M. Schreyer, J. Appl. Phys. 39, p. 4478 (1968).

[17] H. F. McFarlane, K. M. Goff, F. S. Felicione, C. C. Dwight, and D. B. Barber, J. Met. 14, p. 1 (1997).

[18] C. C. McPheeters, E. C. Gay, P. J. Karell, and J. P. Ackerman, J. Met. 49, p. N7 (1997).

[19] B. F. Woodfield, University of California-Berkeley-Thesis, Specific-Heat of High-Temperature Superconductors: Apparatus and Measurement (1995).

[20] J. C. Lashley, Brigham Young University-Thesis, Mechanics of Metals with Phase Changes (2000).

[21] L. Fast, O. Eriksson, B. Johansson, J. M. Wills, G. Straub, H. Roeder, and L. Nördstrom, Phys. Rev. Lett. 81, p. 2978 (1998).

[22] S. Sachdev, Science 288, p. 475 (2000). 


\section{A SUCCINCT ACCOUNT OF PLUTONIUM SAMPLE PREPARATION}

God runs electromagnetics by wave theory on Monday, Wednesday, and Friday, and the Devil runs them by quantum theory on

Tuesday, Thursday, and Saturday.

- Sir William Bragg

4.1 ABSTRACT

4.2 INTRODUCTION

4.3 TECHNIQUES FOR GROWING PLUTONIUM CRYSTALS

4.3.1 Growing crystals from molten plutonium

4.3.2 Gowing plutonium crystals in the solid-state

4.4 LOS ALAMOS PROGRAM FOR GROWING PLUTONIUM CRYSTALS

4.3.1 Purification

4.3.2 Strain-anneal

ACKNOWLEDGMENTS

FURTHER READING

\subsection{ABSTRACT}

This chapter outlines the current experimental situation for the preparation of plutonium samples. Special emphasis is placed on solid-state purification methods and solid-state crystal growth techniques. 


\subsection{INTRODUCTION}

Scientists need large single crystals of plutonium to determine many of the fundamental properties of this metal, such as its electronic structure and atomic vibrational modes. In general, the electronic structure of a crystalline solid governs bonding, magnetism, and elasticity. The vibrational properties become increasingly important as the temperature of the material increases.

Because the electronic structure and vibrational modes of metals reflect the underlying symmetries of the lattice, which is never spherically symmetric, those properties always have a directional dependence. But measurements on polycrystalline samples cannot reveal that directionality because probing a polycrystal is akin to averaging over

all crystal directions. Only a single crystal, as an extended, regular array of unit cells, can yield directional information. But single crystals of plutonium do not currently exist.

A program was recently initiated at Los Alamos to prepare plutonium and then grow large single crystals of gallium-stabilized $\delta$-phase plutonium. Our samples must be prepared from metal of the highest purity because the electronic structure and vibrational modes of plutonium are highly affected by the presence of trace elemental impurities and alloy compositions. The required crystal size depends on the measurement techniques. For example, photoemission spectroscopy measurements of the density of electronic states require crystals on the order of 1 cubic millimeter. Resonant ultrasound spectroscopy measurements of the elastic constants need crystals a few cubic millimeters in size. Finally, inelastic-neutron-scattering experiments, which probe the phonon spectra, require single crystals of plutonium-242 that are as large as 1 cubic centimeter. (Plutonium-242 
has a smaller neutron-absorption cross section than plutonium-239 and is the favored isotope for the scattering measurements.)

In this chapter, we review solidification and solid-state methods for growing plutonium crystals and explain why it is difficult to grow single crystals of the required size. We then discuss our purification techniques and crystal-growth program.

\subsection{TECHNIQUES FOR GROWING PLUTONIUM CRYSTALS}

\subsubsection{Growing Crystals from Molten Plutonium}

The easiest way to obtain single crystals of a material is to grow them from the liquid phase (also called the melt). As the molten metal cools and solidifies, the atoms begin bonding to each other in an orderly fashion because an equilibrium position is reached between attractive and repulsive forces.

It is very difficult, however, to grow single crystals of pure plutonium by simply letting the melt solidify. The pure metal passes through six allotropic phases $\left(\varepsilon, \delta^{\prime}, \delta, \gamma, \beta\right.$, and $\alpha$ ) as it solidifies from the melt and cools to room temperature. In each new phase, the plutonium atoms shift positions to form a new crystalline structure with a different density. Any large crystal grains that develop early in the cooling process become disrupted with each subsequent phase transformation. The result is invariably a polycrystalline solid. (See Figure 4.1.)

In the 1960s, scientists at Argonne National Laboratory grew single crystals of $\alpha$-phase plutonium by allowing the molten material to cool under 55 kilobar of pressure. Under those conditions, plutonium solidifies directly into the $\beta$-phase and transforms to the room-temperature stable $\alpha$-phase at $420^{\circ} \mathrm{C}$, rather than at $112^{\circ} \mathrm{C}$, as it does at ambient 


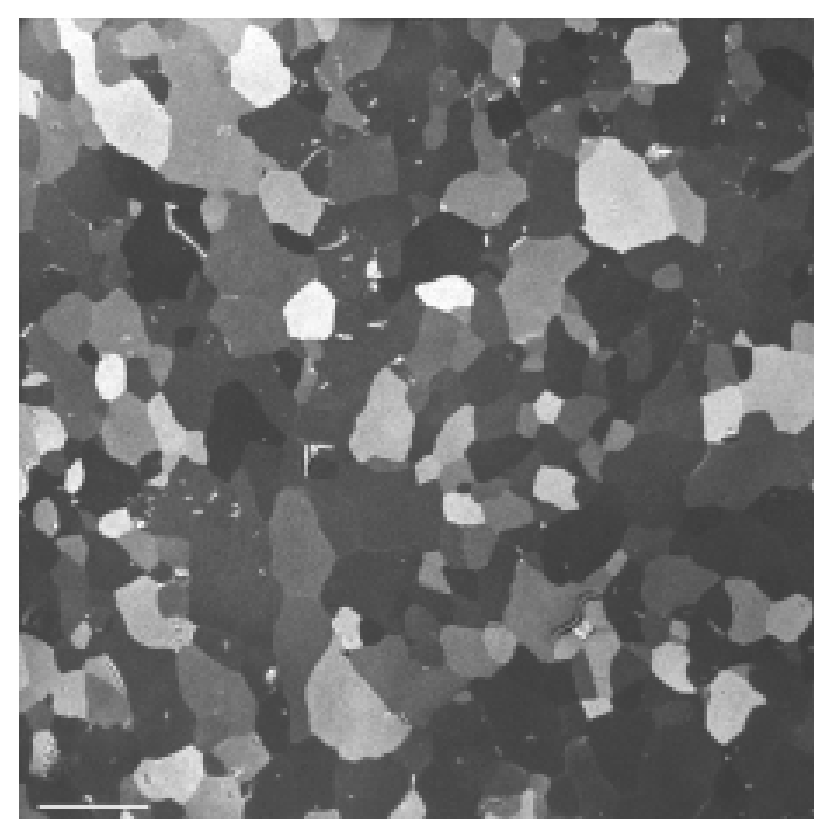

Figure 4.1. Polycrystalline plutonium. Gallium-stabilized, $\delta$-phase plutonium invariably solidifies into a multigrain structure. This sample of $\delta$-plutonium (1 wt \% Ga) was photographed under polarized light in order to make that structure visible. Each grain reflects light differently because the atoms in each grain are aligned in different directions. Efforts are under way to grow grains of plutonium with linear dimensions on the order of $1-10 \mathrm{~mm}$.

pressure. (The higher temperature means that the atoms are relatively mobile as they begin to form $\alpha$-phase grains.) Furthermore, the metal does not change volume in going from the $\beta$ - to the $\alpha$-phase at 55 kilobar. The combination of those advantageous properties allowed researchers to grow large grains, which were then cut from the surrounding matrix and polished into single crystals. Al Arko, who is now at Los Alamos, measured the resistivity and magnetic susceptibility of some of those $\alpha$-phase single crystals.

Also during the 1960s, Roger Moment of Rocky Flats tried to grow large grains of gallium-stabilized $\delta$-phase plutonium. At gallium concentrations between 1 and 2 weight percent (wt \%), the plutonium-gallium alloy exhibits only two allotropes ( $\varepsilon$ and $\delta$ ), and the $\delta$-phase forms at approximately $500^{\circ} \mathrm{C}$. This phase is then thermodynamically stable down to room temperature. 
Moment produced several large grains by the Bridgeman technique: the melt froze and slowly transformed to the $\delta$-phase as it passed through a decreasing temperature gradient. As revealed by x-ray pictures taken with a back-reflection Laue camera, however, all those large grains contained substructure. They were therefore unsuitable for high-quality measurements of physical properties.

One reason for the low grain quality might have been the internal conditions resulting from coring. As discussed in Figure 4.2, when the alloy freezes from the melt or passes through a mixed phase, such as liquid and $\varepsilon,(L+\varepsilon)$, the grains that form have a higher concentration of gallium at the center. The uneven distribution of alloying material introduces strain into the crystal lattice that could detrimentally affect grain growth.

Primarily to avoid coring and other potential problems associated with the $\varepsilon$ - to $\delta$-phase transition, Moment abandoned the Bridgeman technique and focused his attention on growing plutonium grains by strain-anneal methods. Those methods ultimately proved to be successful, and his measurements of the elastic constant are still the only ones made on a single crystal of $\delta$-phase plutonium. Our current program has been guided by Moment's experiences.

\subsubsection{Growing Plutonium Crystals in the Solid State}

Strain-anneal techniques allow crystals to grow entirely within the solid state. During the strain-anneal process, the metal is plastically deformed so that dislocations form within it. The dislocations are disruptions to the minimum-energy state of the perfect lattice and are consequently a form of stored energy. At small plastic strain values (less than 10 percent), approximately 1 to 4 percent of the strain energy is stored within the lattice from dislocations, and the rest is dissipated as heat. 


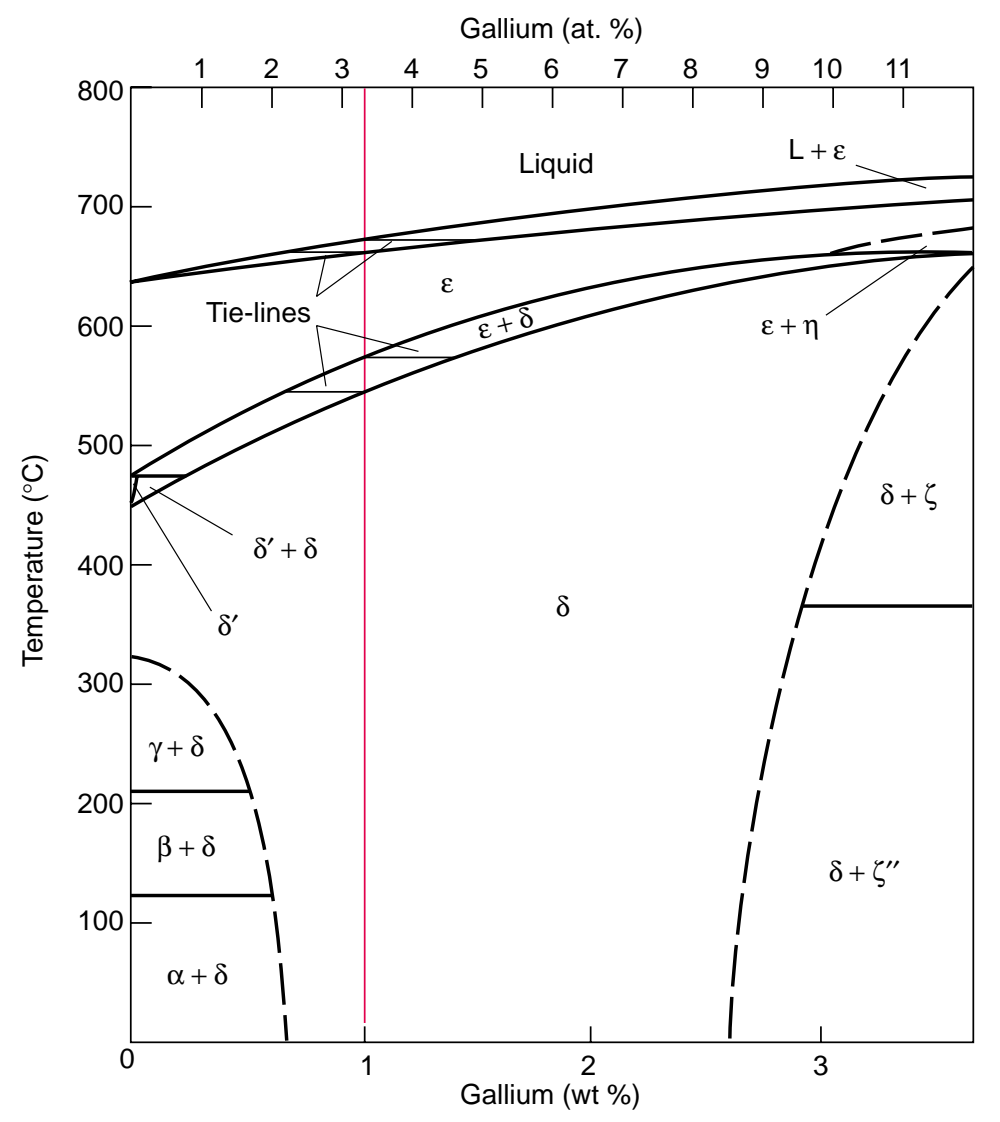

Figure 4.2. The low-gallium composition of the plutonium-gallium binary phase diagram. Pure plutonium has the most allotropes of any element. Following its phase transformations down the leftmost axis, we notice that liquid plutonium (L) solidifies into the $\varepsilon$-phase below $640^{\circ} \mathrm{C}$ and then assumes five more structural phases $\left(\delta^{\prime}, \delta, \gamma, \beta\right.$, and $\left.\alpha\right)$ as it cools to room temperature. Alloying plutonium with small amounts of gallium (less than about $2.5 \mathrm{wt} \%$ gallium) reduces the number of allotropes from six to two. For example, at $1 \mathrm{wt} \%$ gallium, the molten alloy cools by passing through a mixed $(\mathrm{L}+\varepsilon)-$ phase, then a pure $\varepsilon$-phase, then a mixed $(\varepsilon+\delta)$-phase before reaching the $\delta$-phase, which is stable down to room temperature. The first bits of $\varepsilon$-phase material to solidify from the $(L+\varepsilon)$-phase have a higher gallium concentration than the melt. We determine the exact composition by following the first horizontal tie-line in the $(\mathrm{L}+\varepsilon)$-region to the right. Newly solidifying material has less gallium, and thus there are composition gradients within each grain (coring). Coring reoccurs when the alloy passes through the $(\varepsilon+\delta)$-phase.

The stored energy is released when the metal is heated during the first step in the annealing process. The energy drives the formation of new grains in a process called 
recrystallization. Keeping the metal at constant high temperatures (the next step in the annealing process) then allows the recrystallized grains to grow larger through the migration of grain boundaries.

A grain boundary has a surface energy. Smaller grains have proportionally more surface energy than larger ones, so it is energetically favorable to merge smaller grains into larger ones. The boundary migrates as the atoms from one grain shift and become integrated into another. One grain is "consumed" while the other grows. In principle, one grain can dominate, growing steadily larger until the system reaches equilibrium. Grainboundary migration is enhanced at high temperature because the heat increases the mobility of the atoms. As mentioned earlier, specimens containing $1 \mathrm{wt} \%$ gallium can be annealed at temperatures as high as $500^{\circ} \mathrm{C}$ and still remain in the $\delta$-phase (refer to Figure 4.2.)

The strain-anneal technique is clearly advantageous for alloy systems and multiphase materials such as plutonium. Because the temperature is kept within the room-temperature stable phase, there are no phase transformations and no coring effects. Once a large grain forms, its structure can be preserved down to room temperature.

\subsection{LOS ALAMOS PROGRAM FOR GROWING PLUTONIUM CRYSTALS}

\subsubsection{Purification}

Because impurities hamper the mobility of grain boundaries, any crystal-growth methodology hinges on starting with plutonium metal that is free of elemental impurities. Consequently, the first step in our program is to purify the plutonium starting material.

Purification must be done on molten plutonium, but the strong affinity that the liquid exhibits for almost all elements severely complicates the process. The elements that make 
up the container holding the liquid inevitably contaminate the melt. We have solved this problem by using electromagnetic levitation furnaces. These devices, consisting of induction coils and crucibles, are designed to levitate molten material. Any interactions between the crucible and the liquid metal are therefore eliminated. A levitation furnace is described in more detail in Figure 4.3. No other laboratory in the world uses such advanced equipment to purify plutonium.

Our purification technique starts with plutonium metal that has already been purified twice by electrorefining and has then been cast into a rod. We clean the rod's surface to remove any oxides and hydrides that may have formed. At this point, the metal typically contains impurity levels in the range of 500 to 600 parts per million (ppm). Iron, uranium, magnesium, calcium, nickel, aluminum, potassium, and silicon are among the impurities. The rod is placed in the horizontal crucible of the levitation furnace, as shown in Figure 4.3. The induction coil of this furnace is the large disk in the middle of the photo. Only the small section of the rod directly under the induction coil is liquefied and levitated. When we move the crucible, the molten zone passes slowly through the rod in one direction. Impurities that lower the melting point of the metal remain in the liquid zone, whereas those that raise it are driven into the solid material. Because impurities either move with the zone or are pushed in front of it, both kinds are eventually swept to the end of the rod. Repeating this procedure several times leaves the central portion of the rod highly purified.

We use a mass spectrometer with very low detection limits to measure the impurity levels of 78 trace elements. Results to date indicate that zone refining in a levitation furnace reduces impurities from 523 to 174 ppm; uranium accounts for about 70 percent 


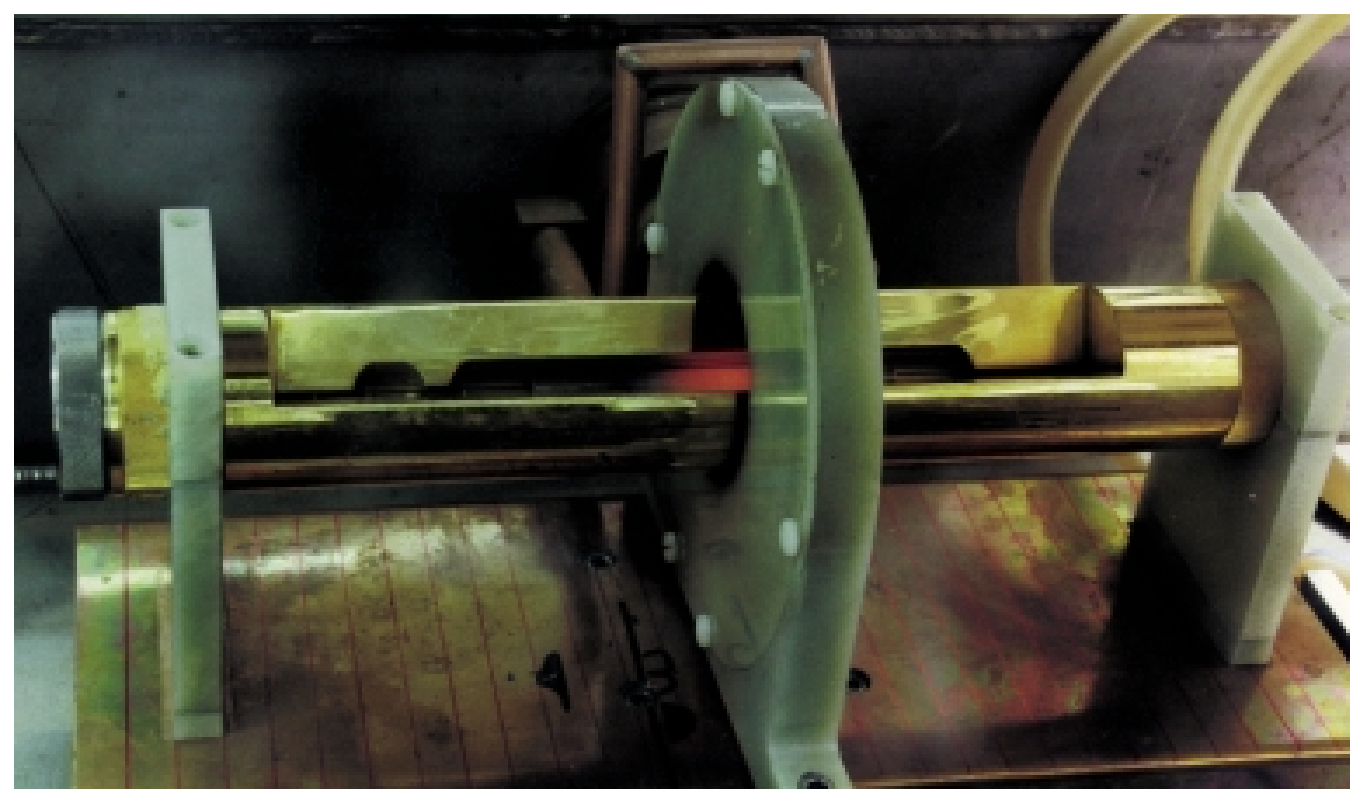

Figure 4.3. Levitation zone-refining furnace. An electromagnetic-levitation furnace consists of a crucible that holds the sample and an induction coil. As shown in the schematic on the right, radio-frequency power running through the coil induces currents to flow in the crucible. The current generates a magnetic field $M_{c}$ that induces eddy currents to flow in the sample, which heats up and melts. But the eddy currents generate a secondary magnetic field in the direction opposite to $\mathrm{M}_{\mathrm{c}}$. Magnetic repulsion causes a zone of

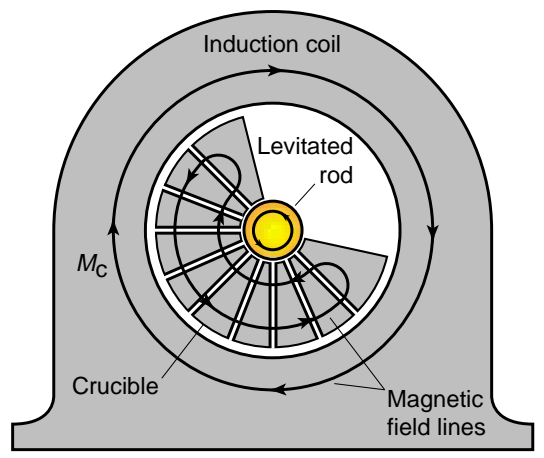
liquefied metal to levitate a small distance from the crucible walls. The photo shows a stainless steel rod loaded in the horizontal crucible. The glowing region under the induction coil is the levitated molten zone, and moving the crucible causes the zone to sweep through the rod. Elements that lower the melting point of the material travel with the zone, while those that raise the melting point diffuse into the solid. In either case, impurities are swept to the end of the rod, which is then cut off. For our program, we purify rods of $\alpha$-phase plutonium, rather than $\delta$-phase, to avoid complications stemming from the addition of gallium.

of that remainder. Also, we have not detected any crucible material in our sample, which indicates a complete lack of plutonium-crucible interactions.

The purified rod then undergoes vacuum distillation in a vertical levitation furnace, a process that purges the rod of americium and interstitial gas impurities. The zone-refined plutonium is placed under reduced pressure $\left(10^{-7}\right.$ torr $)$ in a cylindrical, vertical crucible. 
An induced high current circulates through the entire metal rod, which melts and levitates. Because americium and other impurities have a high vapor pressure, they distill away. We also add enough gallium during this stage to produce the plutonium-gallium alloy. Sudden removal of furnace power lets the molten alloy drop into a chilled copper crucible, where it quickly solidifies. (See Figure 4.4.) Because the solidification is so rapid, coring is minimized, and we obtain an as-cast density of 15.94 grams per cubic centimeter $\left(\mathrm{g} / \mathrm{cm}^{3}\right)$, which is only slightly higher than the theoretical $\delta$-phase density of $15.81 \mathrm{~g} / \mathrm{cm}^{3}$. (A small amount of coring creates some high-density $\alpha$-phase plutonium that increases the overall density.) Recent results obtained from vacuum distillation show that the americium could be removed almost entirely. Several weeks after distillation, the

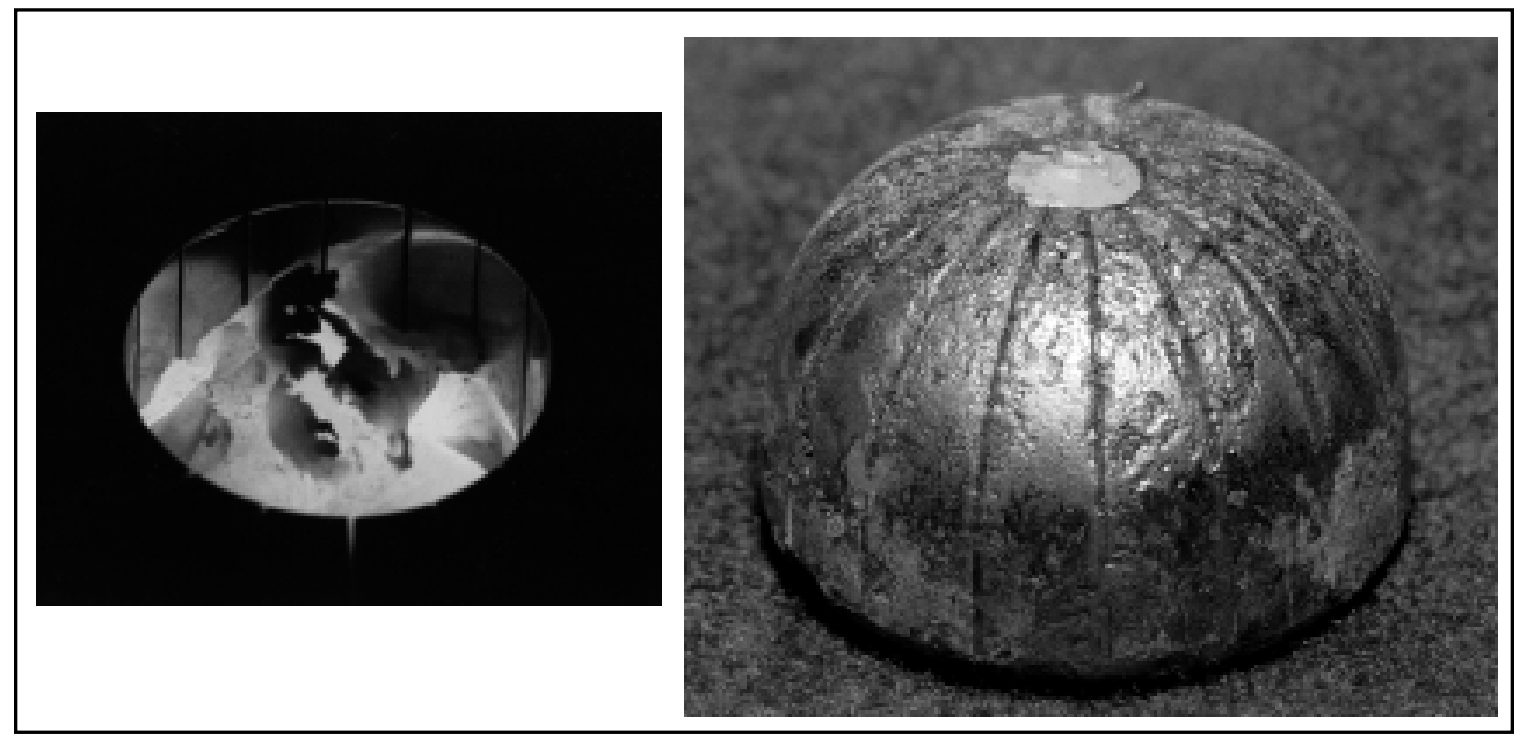

Figure 4.4. Vacuum distillation of plutonium metal. In the photo on the left, molten plutonium metal is held suspended in the middle of a vertically-oriented crucible by an electromagnetic field. Americium and gas impurities boil off. Enough gallium has been added to stabilize the $\delta$-phase plutonium upon solidification. The photo on the right shows a half sphere of gallium-stabilized $\delta$-phase plutonium that was produced by vacuum distillation in a levitation furnace. The lines on the surface reveal where the plutonium solidified to the shape of the mold. Samples for growing crystals were fabricated from this specimen. 
measured impurity level was $1.5 \pm 0.3 \mathrm{ppm}$. During that time, however, some of the plutonium-241 that was present in the starting material decayed to americium-241. What we detected was simply the newly created americium.

The ability to make plutonium samples that are essentially free of americium is critically important to the Laboratory. Such a sample is needed for an experiment to measure the $(\mathrm{n}, 2 \mathrm{n})$ cross section of plutonium-239. The cross section can be used to infer the neutron spectrum in an exploding weapon and is therefore a central concern of the Science-Based Stockpile Stewardship program. The results of the nuclear cross-section measurements are reported in Appendix 1.

\subsubsection{Strain-anneal}

Using ultrapurified material, we set up a series of experiments to grow large grains of $\delta$-phase plutonium. Our starting material was a set of 3-millimeter-thick polycrystalline disks ( $1 \mathrm{wt} \%$ gallium) that had been cut from a homogenized ingot (average grain size of 30 micrometers). After rigidly fixing each disk's circumference into a die, we deformed the disks over a hemispherical punch at room temperature, as in Figure 4.5(a).

This setup made it easy to strain the material uniformly in both the radial and circumferential directions (balanced-biaxial strain). The total, true strain on the surface is described by the three diagonal components of the true strain tensor: the radial component $\left(\varepsilon_{1}\right)$, a circumferential component $\left(\varepsilon_{2}\right)$, and a through-thickness component $\left(\varepsilon_{3}\right)$. Only two of those components are independent. The strain peaks at the pole and goes to zero at the periphery. Because the volume of the material is conserved, the disk also becomes thinner. At small plastic deformations, a uniform through-thickness compressive strain is also achieved that varies with the radius. 
With the strain-anneal technique, the largest grains are produced if the metal is strained a "critical" amount and then annealed. If strained too little, the metal does not acquire enough dislocations (that is, stored energy) to promote recrystallization, but if strained too much, it acquires so many dislocations that grain refinement occurs. We wanted to achieve this critical strain at the center of the disk so that the grain would then grow out uniformly in all directions. Unfortunately, the critical strain depends on many factors, including the initial sizes and orientations of the grains and the manner in which the load is applied to the bulk material. That is why the critical strain can be determined only empirically for each type of sample.

After a 90 -hour isothermal anneal at $480^{\circ} \mathrm{C}$ and reduced pressure, grains of 1-2 millimeters in length were produced. The largest grain was located about $6 \mathrm{~mm}$ from the center of the disk. We estimated a critical strain of about 4 percent. Analysis of the disc surface at the location of the largest grain yielded components of the strain tensor (corresponding to the critical strain for this material) of $\varepsilon_{1}=0.02$ and $\varepsilon_{2}=0.02$. Having some sufficiently large grains available to us, we extracted from the disk a small section containing one such grain-see Figure 4.5(b).

We have also experimented with a second method for growing crystals. Liquid plutonium-gallium alloy is dropped out of the magnetic field of a levitation furnace and is chill-cast in a mold shaped like a long, thin cone. The microstructure of this chill-cast plutonium consists of very fine, small acicular (or needlelike) grains $10 \times 25$ micrometers in size. 

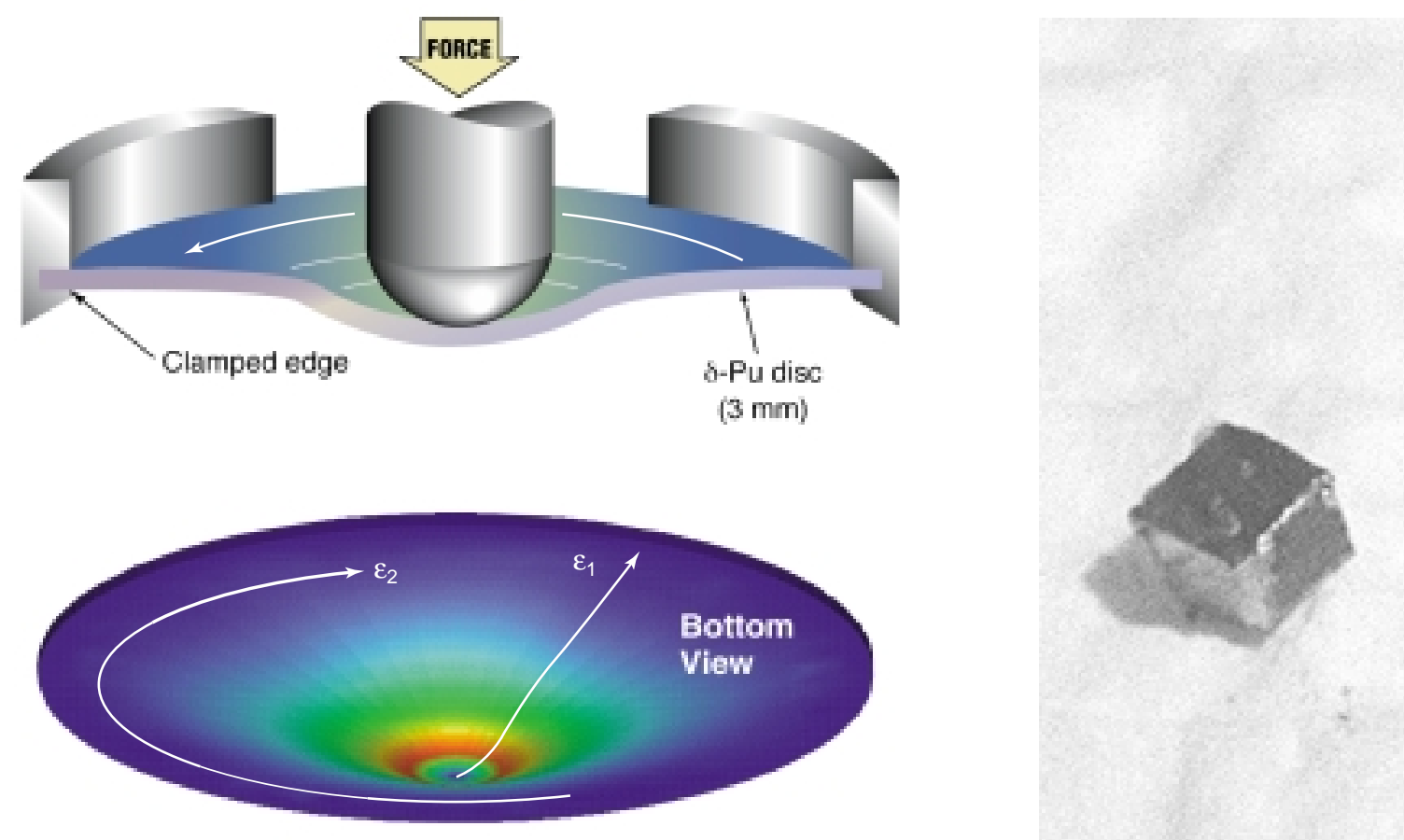

Figure 4.5. Biaxial stress in a plutonium disk. (a) A 3-mm-thick disk of gallium-stabilized $\delta$-phase plutonium is stretched in two dimensions with a hemispherical punch. The magnitude of the strain decreases in the radial direction, from the pole to the edge. A strain gradient, described by $\varepsilon_{1}$ is therefore generated and is shown here by different bands of color. A second strain gradient is produced as the disk becomes thinner. (b) After having been annealed isothermally at $480^{\circ} \mathrm{C}$ for 90 hours, the disk contained several newly grown, large $(1 \mathrm{~mm})$ plutonium grains. The cube shown above contains one of those grains.

We anticipate that the conical geometry of this chill-cast sample and the fine grain size will help promote grain growth. Because the tip of the cone is so small, the number of grains competing to grow there is reduced. Hopefully, a single grain will grow at the expense of the others once we begin a strain-anneal process. Because the grain size in the rest of the sample is so small, there is a lot of surface energy available to help drive that grain growth, and the growing "seed" grain could continue to expand into the cone.

Ideally, the large volume at the top of the cone would consist of a single grain from which precisely oriented samples could readily be cut. 
The lack of large single crystals is currently the only obstacle that prevents researchers from obtaining high-quality data on the fundamental solid-state properties of $\delta$-phase plutonium. By applying several variations of the strain-anneal technique to $\delta$-phase plutonium, we have enjoyed some success in growing millimeter-sized grains. Our future work is aimed at developing crystal-growing methods with repeatable results.

\section{ACKNOWLEDGMENTS}

I would like to thank Michael Blau and Roger Moment. I would also like to thank the entire staff of Los Alamos Science. I would like to thank Michael Stout, David Embury, Manuel Lovato, Ramiro Pereyra, Jim Smith, Larry Hults, Jason Cooley, and Karl Staudhammer of the Materials Science Division. Special thanks to Floyd Rodriguez, Fred Hampel, and Kenny Vigil for laboratory assistance, and to Mick Greenbank and Larry Vaughn for photography and induction support, respectively. This work was carried out at the Los Alamos National Laboratory under the auspices of the Department of Energy. 


\section{FURTHER READING}

[1] Goss, A. J., 1963, In The Art and Science of Growing Crystals, J. L. Gilman, ed., New York: John Wiley and Sons.

[2] Hecker S. S., 1976, Scientific American, November issue.

[3] Hecker, S. S., 1976, Los Alamos National Laboratory document LA-UR-76-1413.

[4] Lander G. H., E. S. Fisher, and S. D. Bader, 1994, Adv. Phys. 43:1.

[5] Lashley, J. C., M. S. Blau, K. P. Staudhammer, and R. A. Pereyra, 1999, J. Nucl. Mater. 274: 315.

[6] Liptai, R. G., L. T. Lloyd, and R. J. Friddle, 1967, J. Phys. Chem. Solids 1: 573.

[7] Wallace, D. C. 1972, Thermodynamics of Crystals, New York: Dover Publications, Inc. 
IN SITU PURIFICATION, ALLOYING, AND CASTING OF PLUTONIUM METAL

Plutonium is at odds with itself.

- J. L. Smith

\subsection{ABSTRACT \\ 5.2 INTRODUCTION \\ 5.3 EXPERIMENTAL PROCEDURE \\ 5.4 EXPERIMENTAL RESULTS \\ 5.5 CONCLUSION \\ ACKNOWLEDGMENTS \\ REFERENCES}

\subsection{ABSTRACT}

Plutonium metal that has been double ER (electro refined) was further purified by zone refining, using a floating molten zone to minimize the introduction of impurities. The temperature of the molten zone was $1023 \mathrm{~K}$, and the atmosphere was $10^{-5} \mathrm{~Pa}$. A total of ten zone refining passes were made at a travel rate of $1.5 \mathrm{~cm} \mathrm{hour}^{-1}$. There were 19 elements reduced to quantities below the minimum detectable limits (MDL) by zone refining, while the elements $\mathrm{P}, \mathrm{K}$, and $\mathrm{W}$ were significantly reduced. The zone-refined metal was then used to prepare tapered specimens for single-crystal growth experiments. Specifically, ${ }^{241} \mathrm{Am}$ (a plutonium daughter product) was distilled from Pu metal by levitating Pu metal with 1 wt \% Ga in the melt in a Crystallox vertical electromagnetic 
levitation crucible at $10^{-5} \mathrm{~Pa}$. The $\mathrm{Pu}$ is alloyed with $\mathrm{Ga}$ to stabilize the $\delta$-phase (fcc symmetry) upon solidification. The sample showed $10 \pm 25 \mu \mathrm{m}$ acicular grains with an as-cast density of $15.938 \mathrm{~g} \mathrm{~cm}^{-3}\left( \pm 0.002 \mathrm{~g} \mathrm{~cm}^{-3}\right)$.

\subsection{INTRODUCTION}

Plutonium metal processing at Los Alamos includes a molten salt extraction (MSE) step, followed by one or two ER runs. In these two processes, Pu metal shows a strong affinity for crucible materials and anode/cathode materials. For example, it has been reported that two of the principal metallic impurities present in $\mathrm{Pu}$ produced by the ER process are $\mathrm{W}$ and $\mathrm{Fe}$, which are caused by the interaction between $\mathrm{Pu}$ and the container material $[1,2]$. Similarly, it has been shown that $\mathrm{Al}, \mathrm{Cr}$, and $\mathrm{Ni}$ are usually reduced to low levels by the ER process, but they seem to be affected by the impurity content of the anode material or by the electrolysis conditions [3].

The daughter products that grow into Pu over time are always present as impurities, and they add a new degree of complexity to purification. The principal daughter products of $\mathrm{Pu}$ are isotopes of uranium and americium. The ${ }^{235} \mathrm{U}$ nuclei grow into $\mathrm{Pu}$ from the $\alpha$-decay of the ${ }^{239} \mathrm{Pu}$ nuclei, and ${ }^{241} \mathrm{Am}$ is present from the $\beta$-decay of the ${ }^{241} \mathrm{Pu}$ nuclei [4]. Typically, the ${ }^{235} \mathrm{U}$ nuclei grow into $\mathrm{Pu}$ at $30 \mathrm{ppm}$ per year, and ${ }^{241} \mathrm{Am}$ grows in at $120 \mathrm{ppm}$ per year depending on the initial ${ }^{241} \mathrm{Pu}$ composition. Therefore, the initial $\mathrm{Pu}$ isotopic composition and the time interval between sample preparation and analysis are important factors that can affect the analytical results.

Understanding the effects of $\mathrm{Pu}$ trace impurities on the intrinsic properties and on grain growth has been a primary goal of our research. Since the discovery of plutonium in 
1941, the measured intrinsic properties of the metal have shown an extreme sensitivity to alloying and to the presence of trace elemental impurities [5]. For example the negative coefficient of thermal expansion has been a hallmark of $\delta$ - and $\delta^{\prime}$-plutonium. However, the thermal expansion becomes less negative and finally becomes positive with increasing alloying additions of aluminum, gallium, zinc, zirconium, indium, and cerium [6,7]. Conversely, the lower-temperature phases of unalloyed plutonium all exhibit positive coefficients of thermal expansion [8].

The large effects that small impurity concentrations exert on the thermal expansion behavior and on the fcc stability field highlight the importance of a thorough characterization of trace elemental impurities. Historically, the total impurity numbers reported in $\mathrm{Pu}$ metal have been reported on a relatively small set, usually 20 to 40 impurity elements. Our efforts are directed at revisiting fundamental property measurements and grain growth with high-purity plutonium metal. The trace impurities reported here are measured by inductively coupled plasma mass spectrometry (ICP-MS) and atomic emission spectroscopy (ICP-AES). These methods detect a suite of 75 trace impurities, and the total impurity levels are quantified to the $100-\mathrm{ppm}^{1}$ level, which corresponds to $99.99 \mathrm{wt}$ $\%$ metal. We report here novel methods for Pu alloy synthesis for specimens that will be used in subsequent grain growth experiments. In the first of a three-step process, doubleER Pu is vacuum-cast into rods. The Pu rods are further purified by floating zone refining, then by levitation distillation at reduced pressure. The floating zone refining method targets metals and metalloids, then the levitation distillation method targets ${ }^{241} \mathrm{Am}$. In the third step, Pu metal is cast directly from the electromagnetic field into tapered specimens.

\footnotetext{
${ }^{1}$ Concentration expressed $\mathrm{ppm}$ is defined as $\mu \mathrm{g} / \mathrm{g}$.
} 
Tapered castings serve three purposes: first, specimens can be alloyed in an electromagnetic field, thereby minimizing the introduction of impurities; second, chill casting directly from the electromagnetic field produces a fine grain size, thereby reducing the concentration gradients of $\mathrm{Ga}$; and lastly, the specimens are cast into the correct geometry for subsequent grain growth experiments, thereby eliminating the need for machining. It was anticipated that using a combination of a floating molten zone with the ability to measure 75 trace elements would prove beneficial. It was also anticipated that a fine grain size would eliminate or significantly reduce the coring of $\mathrm{Ga}$. It has been reported that Ga coring and the presence of insoluble impurities at the grain boundaries are two factors that exert detrimental effects on grain growth $[9,10]$.

\subsection{EXPERIMENTAL PROCEDURE}

All work is carried out in a glove box under an argon or vacuum atmosphere. Starting material for the initial series of runs was double-ER Pu that has been cast into rods having a total impurity level of $590 \mathrm{ppm}$ (see table 5.1, first column). The ER process used a feed of recycled Pu alloys, and all specimens had a density $<19 \mathrm{~g} / \mathrm{cm}^{3}$. The double-ER rods were further purified by passing a $10 \mathrm{~mm}$-wide molten floating zone ten times through a cast rod at a travel rate of $1.5 \mathrm{~cm} / \mathrm{h}$ at $10^{-5} \mathrm{~Pa}$. Zone refining was done at reduced pressures with a floating molten zone to take advantage of volatile impurities. The temperature of the molten zone was $750^{\circ} \mathrm{C}$, which was measured using an infrared thermometer.

The floating molten zone was induction melted using a radio-frequency-powerinduced electric current. The crucible acts as a transformer inducing a current in the $\mathrm{Pu}$ 
sample in a direction opposite to the current in the crucible. Magnetic fields in the crucible and the plutonium are opposed, causing repulsion and levitation of the plutonium a small distance from the crucible walls. The induction parameters used were $50-\mathrm{kHz}$ frequency at $20 \mathrm{~kW}$ of power. After each pass the thin oxide layer was removed with a stainless steel wire brush. Core samples were removed for analysis using a cobalt drill bit powered with a variable speed drill motor under an atmosphere of argon. The final trace element analysis of zone-refined rods is shown (Table 5.1, second column).

The zone-refined rod, weighing approximately $240 \mathrm{~g}$, was placed in a water-chilled copper crucible along with sufficient gallium to make a $1 \mathrm{wt} \%$ alloy. The Crystallox levitation crucible was in a vertical position. The bottom of the crucible was fitted with a 1-cm tapered Mycor ceramic mold (Figure 5.1). The vertical crucible operates in the same manner as the zone refining crucible described above with the exception of the induction parameters. Specifically, the parameters used for the levitation distillation were a variable frequency $2-3 \mathrm{kHz}$ operated at $40 \mathrm{~kW}$, and the $\mathrm{Pu}$ and $\mathrm{Ga}$ were induction melted at $800^{\circ} \mathrm{C}$. The $\mathrm{Pu}$ and $\mathrm{Ga}$ mixture was levitated in the melt for $30 \mathrm{~min}$ to ensure thorough mixing by the induction currents. Gallium was purchased in ingot form (99.9999\%) from Alpha Aesar and was used without further purification. The Ga was added in excess to correct for volatilized Ga. A water-cooled copper condenser was located above the crucible to trap the volatile ${ }^{241} \mathrm{Am}$. Next, the alloy was chill-cast directly from the magnetic field into a ceramic mold at the bottom of the crucible by reducing the furnace power. The cooling rates of the Pu castings are measured using an infrared thermometer. 


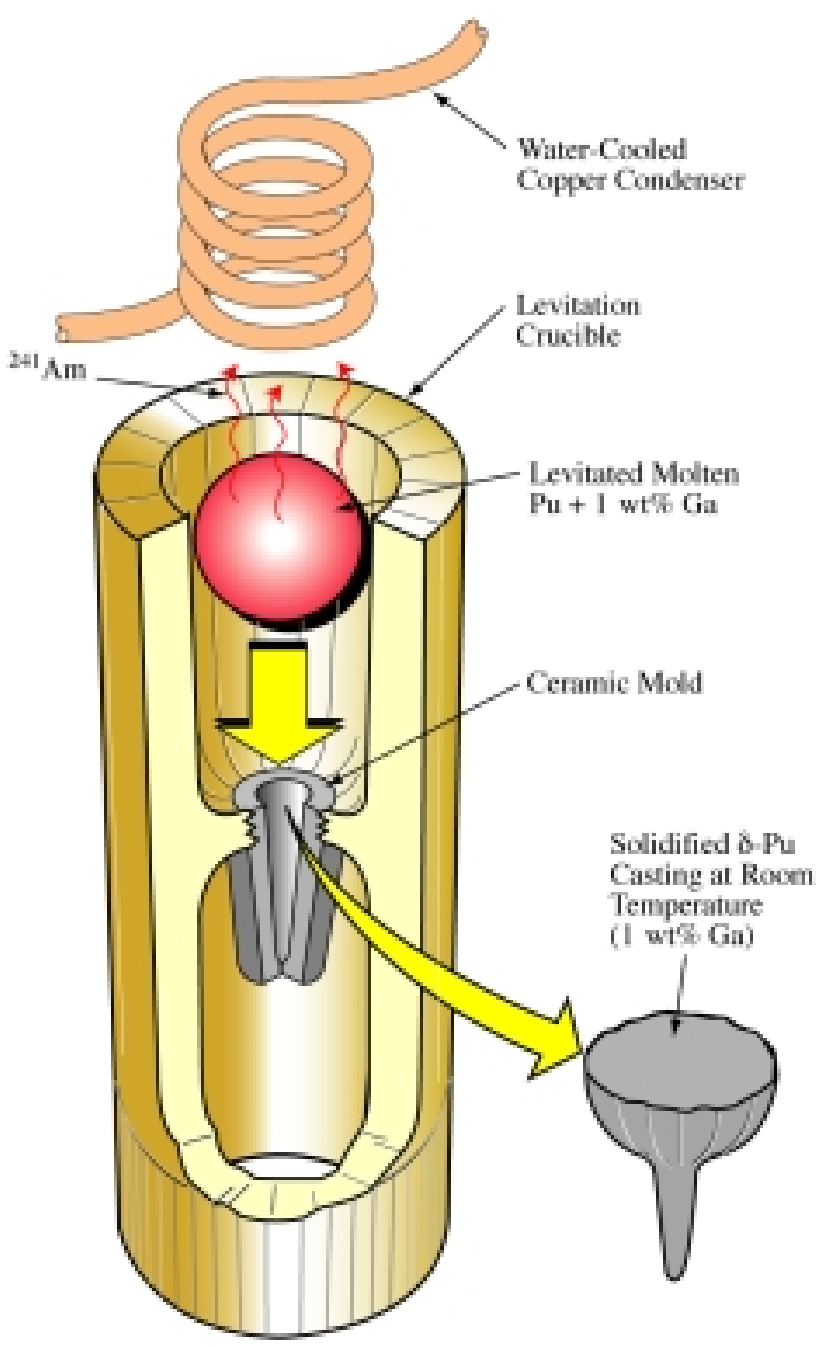

Figure 5.1. In situ americium distillation, alloying, and chill-casting step. A mixture of a$\mathrm{Pu}$ and $\mathrm{Ga}$ are added in a levitation crucible. The mixture is heated to the melt while suspended in a magnetic levitation field. As the $\mathrm{Ga}$ mixes with the $\mathrm{Pu}$ in the melt, it will stabilize the $\delta$-phase upon solidification. While in the melt, ${ }^{241} \mathrm{Am}$ distilled from the $\mathrm{Pu}$ is collected onto a water-cooled condenser. Next, the furnace power is reduced, and $\mathrm{Pu}$ is cast directly from the electromagnetic field into a ceramic mold.

Plutonium castings are prepared for metallographic examination by cutting on a slow-speed diamond saw using a PF-5060 (freon substitute) lubricant under an argon atmosphere. Samples are mounted in Epon 815 epoxy, and the epoxy is allowed to dry for 12 hours. Samples are then mechanically polished on polishing wheels starting at 230-grit paper and ending up with a $0.25-\mu \mathrm{m}$ diamond paste. Final polishing is done in an electro- 
chemical cell in a $10 \%$ solution of nitric acid in $90 \%$ dimethyl formamide. The samples are electrochemically etched in the same solution to relieve the grain structure. The samples are then photographed at various magnifications. The standard ASTM lineintercept method was used to determine average grain size. Density was measured by the density immersion method at room temperature in an immersion bath of bromobenzene.

\subsection{EXPERIMENTAL RESULTS}

The trace elemental impurities in double electrorefined plutonium metal determined by ICP-MS and ICP-AES are listed in Table 5.1, first column. The major impurities present in the ER material were $\mathrm{Na}, \mathrm{K}, \mathrm{Fe}, \mathrm{Ta}, \mathrm{W}$, and U. High concentrations of $\mathrm{Na}$ and $\mathrm{K}$ are a result of salts that become trapped during the ER process. The presence of Fe, Ta, and $\mathrm{W}$ is caused by the interactions of $\mathrm{Pu}$ with the anode/cathode used in the current $\mathrm{ER}$ process. High uranium concentrations are a result of using older Pu for the initial ER feed.

The trace impurity levels after zone refining are given in Table 5.1, second column. Results from zone refining have shown the reduction of impurities in double-electrorefined and vacuum-cast unalloyed plutonium from a total of $590 \mathrm{ppm}( \pm 88 \mathrm{ppm})$ impurities to $174-\mathrm{ppm}$ ( $\pm 26 \mathrm{ppm})$ through floating zone refining. A total of 19 elements were reduced to quantities below the minimum detectable limits by zone refining, while levels of $\mathrm{P}, \mathrm{K}$, and $\mathrm{W}$ were significantly reduced. The elements that were removed by the zone refining process were $\mathrm{Li}, \mathrm{Be}, \mathrm{Na}, \mathrm{Ca}, \mathrm{Mn}, \mathrm{Fe}, \mathrm{Co}, \mathrm{Ni}, \mathrm{Ge}, \mathrm{Rb}, \mathrm{Nb}, \mathrm{Pd}, \mathrm{Ag}, \mathrm{In}, \mathrm{Sn}, \mathrm{Cs}, \mathrm{Ta}$, $\mathrm{Au}$, and Ce. Uranium accounts for $110 \mathrm{ppm}$ of the measured $174 \mathrm{ppm}$ in the zone refined $\mathrm{Pu}$. Presumably, this is a result of a very small slope in the low uranium concentration portion of the liquid-solid curve in the plutonium-uranium phase diagram [12]. 
Table 5.1. Table of Pu Impurities. Trace elemental impurities present in doubleelectrorefined plutonium. Analysis was performed by ICP-MS for 75 elements. Elements that do not appear are below their respective method detection limit (MDL), and hence they cannot be quantified. The relative standard deviation of the analysis by the ICP method was $\pm 15 \%$.

\begin{tabular}{|c|c|c|c|}
\hline Impurity Element & $\begin{array}{l}\text { Double Electro- } \\
\text { Refined } \\
\text { Concentration } \\
\text { (ppm) }\end{array}$ & $\begin{array}{c}\text { Zone Refined } \\
\text { Concentration } \\
(\mathrm{ppm})\end{array}$ & $\begin{array}{c}\text { Minimum } \\
\text { Detection Limit } \\
(\mathrm{MDL}) \\
(\mathrm{ppm})\end{array}$ \\
\hline LITHIUM & 2.70 & $<0.40$ & 0.40 \\
\hline BERYLLIUM & 0.80 & $<0.18$ & 0.18 \\
\hline SODIUM & 115.00 & $<8.80$ & 8.80 \\
\hline PHOSPHORUS & 95.00 & 7.00 & 5.60 \\
\hline POTASSIUM & 95.00 & 40.00 & 14.00 \\
\hline CALCIUM & 3.70 & $<0.50$ & 0.50 \\
\hline CHROMIUM & 3.10 & 4.20 & 0.13 \\
\hline MANGANESE & 1.00 & $<0.07$ & 0.07 \\
\hline IRON & 61.00 & $<60.00$ & 60.00 \\
\hline COBALT & 1.00 & $<0.10$ & 0.10 \\
\hline NICKEL & 2.10 & $<0.40$ & 0.40 \\
\hline COPPER & 1.90 & 0.80 & 0.13 \\
\hline GERMANIUM & 5.00 & $<0.32$ & 0.32 \\
\hline RUBIDIUM & 1.00 & $<0.11$ & 0.11 \\
\hline NIOBIUM & 1.00 & $<0.40$ & 0.40 \\
\hline SILVER & 1.00 & $<0.13$ & 0.13 \\
\hline PALLADIUM & 1.00 & $<0.07$ & 0.07 \\
\hline CADMIUM & 1.00 & 0.05 & 0.04 \\
\hline INDIUM & 1.00 & $<0.06$ & 0.06 \\
\hline TIN & 1.00 & $<0.03$ & 0.30 \\
\hline CESIUM & 1.00 & $<0.09$ & 0.09 \\
\hline CERIUM & 1.00 & $<0.04$ & 0.04 \\
\hline HAFNIUM & 1.00 & 0.18 & 0.05 \\
\hline TANTALUM & 16.00 & $<2.20$ & 2.20 \\
\hline TUNGSTEN & 61.00 & 10.00 & 2.20 \\
\hline RHENIUM & 1.00 & 0.20 & 0.14 \\
\hline GOLD & 1.00 & $<0.36$ & 0.36 \\
\hline LEAD & 2.40 & 1.70 & 0.08 \\
\hline URANIUM & 121.00 & 110.00 & 0.07 \\
\hline TOTAL & $590( \pm 88)$ & $174( \pm 26)$ & \\
\hline
\end{tabular}

The elements below the MDL in both electrorefined and zone refined are: $\mathrm{B}, \mathrm{Mg}, \mathrm{Al}, \mathrm{Si}, \mathrm{S}, \mathrm{Sc}, \mathrm{Ti}$, V, Zn, As, Se, Sr, Y, Zr, Mo, Tc, Ru, Sb, Te, Ba, La, Os, Ir, Pt, Hg, Tl, Bi, Po, Fr, Ra, Ac, Pr, Nd, $\mathrm{Pm}, \mathrm{Sm}, \mathrm{Eu}, \mathrm{Gd}, \mathrm{Tb}, \mathrm{Dy}, \mathrm{Ho}, \mathrm{Er}, \mathrm{Tm}, \mathrm{Yb}, \mathrm{Lu}, \mathrm{Th}$, and Pa. In the as-cast specimen the ${ }^{241} \mathrm{Am}$ concentration was $1.50 \mathrm{ppm}( \pm 0.30 \mathrm{ppm})$ as measured by alpha-ray spectroscopy. The impurity results of ER metal reported in the first column differ from impurity results reported in ER Pu metal done in the 1960 era. Although the ER process has not changed significantly in the past 3040 years, the analytical chemistry methods have. For example, the ER metal results reported here are based on 75 elements with lower detection limits for most elements. Earlier ER metal results are based on a total 20-35. Another source of difference is that the original Pu metal used for feed in the current ER process consisted of recycled alloys that are old relative to the feed material used in the 1960 era. The feed metal used in the 1960 era had not aged significantly between reactor production and processing. Uranium is the primary impurity reported in the first column and is a direct result of using older recycled material as an ER feed. 
The castings show fine acicular grains with an average grain size of $10 \times 25 \mu \mathrm{m}$ (Figure5.2) with an average density of $15.938 \mathrm{~g} / \mathrm{cm}^{3}( \pm 0.002)$. The standard density of a fully homogenized $\delta$-Pu ( $1 \mathrm{wt} \%$ Ga alloy) specimen is $15.70 \mathrm{~g} / \mathrm{cm}^{3}$ [12]. Presumably, our density is slightly higher because of a small amount of the higher-density $\alpha$-phase that has been retained. This was confirmed by optical metallography. In particular, the lighter areas around the grain boundaries in Figure 5.2 are small pockets of the low-temperature $\alpha$-phase of plutonium. Darker areas show the $\delta$-phase and are rich in gallium. Structures of this type are desirable for grain growth studies because homogenization time is minimized as a result of a small grain size.

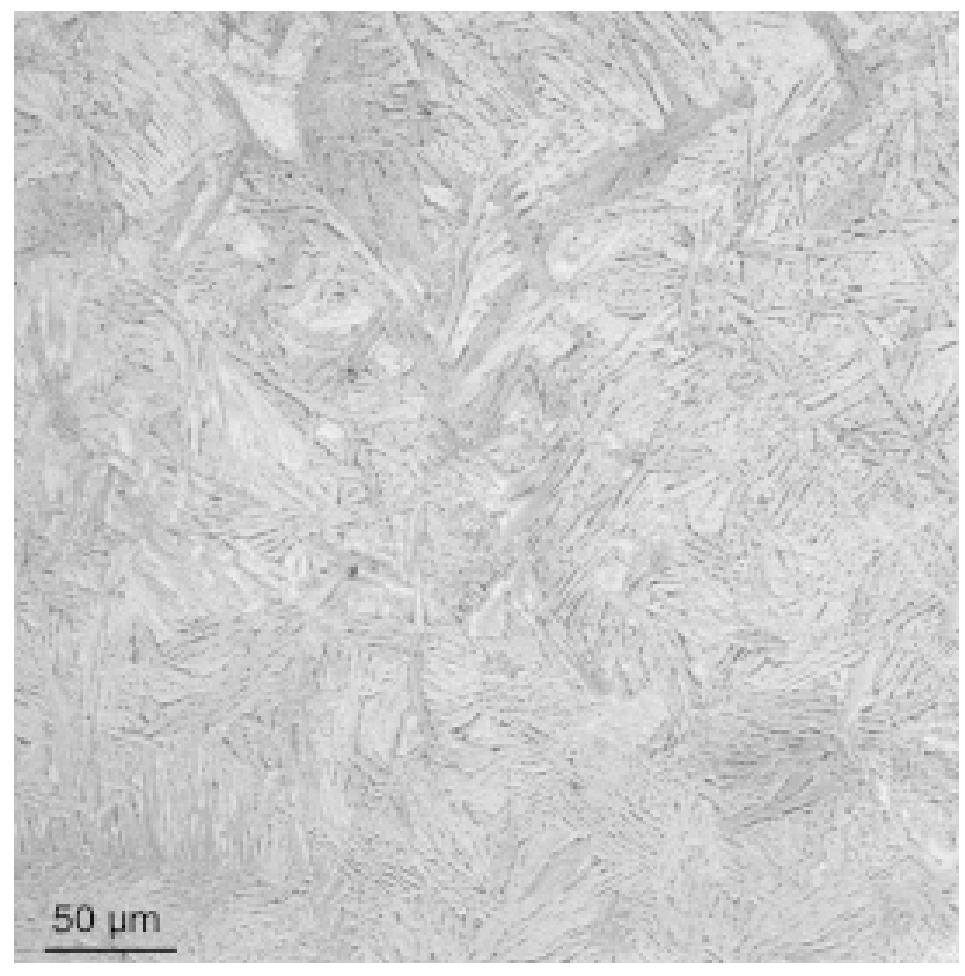

Figure 5.2. Chill-Cast Pu Microstructure. A microstructure of the chill-cast plutonium sample reveals small acicular grains $10 \times 25 \mu \mathrm{m}$ in size. The darker areas represent gallium-rich regions. Similarly, the light areas (around the grain boundaries) are areas that are deficient in gallium and represent the higher-density $\alpha$-Pu phase. 


\subsection{CONCLUSIONS}

The combination of floating zone refining with an in situ distillation, alloying, and casting technique has produced good specimens used for subsequent grain growth experiments. Based on the experimental data presented above, the following conclusions are summarized:

1. ER metal containing $590 \mathrm{ppm}$ can be further purified by floating zone refining to a total impurity level of $174 \mathrm{ppm}$.

2. Chill-cast specimens are obtained in the correct geometry for subsequent grain growth experiments, thereby eliminating the need to perform additional machining steps.

3. Rapid solidification rates of $373 \mathrm{~K} \mathrm{~min}^{-1}$ minimize $\mathrm{Ga}$ coring and produce a small grain structure with a density of $15.938 \mathrm{~g} \mathrm{~cm}^{-3}$.

4. Molten plutonium does not react with containment vessels, and compared to current metal processing methods, electromagnetic levitation minimizes waste generation.

5. This density of the castings, very close to that of fully homogenized $\delta$-Pu, indicates that a small amount of $\alpha-\mathrm{Pu}$ has formed, which was confirmed by optical metallography.

6. In the context of plutonium's unusual properties, the combination of electromagnetic levitation and casting directly from the electromagnetic levitation field has been a robust method to enhance the current MSE and ER processes for the preparation of high-purity research samples.

\section{ACKNOWLEDGMENTS}

Michael Blau, Karl Staudhammer, and Ramiro Pereyra are thanked for their contributions to the work presented in this chapter. Roger Moment, Michael Stevens, and

Jim Smith are thanked for many useful discussions. This work was performed at the Los Alamos National Laboratory under the auspices of the Department of Energy. 


\section{REFERENCES}

1. J. A. Leary and L. J. Mullins, LA-3356-MS, Los Alamos Scientific Laboratory (1965).

2. L. J. Mullins and J. A. Leary, I \& EC Process Des. and Dev. 4(4) (1965), 394.

3. B. Blumenthal and M. B. Brodsky, "The preparation of High Purity Plutonium" in PLUTONIUM 1960 (Cleaver-Hume Press Ltd., London).

4. G. T. Seaborg and W. D. Loveland, The Elements Beyond Uranium (John Wiley \& Sons, INC., New York, 1990).

5. J. E. Selle and B. B. Lawton, RFP-4327, EG\&G Rocky Flats Report (1991).

6. E. A. Kmetko, "The band structure, specific heat, magnetic susceptibility, and anomalous thermal expansion of delta-plutonium" in Proceedings of the Third International Conference on Plutonium (Cleaver-Hume Press Ltd., London) (1965).

7. F. W. Schonfeld and R. E. Tate, Los Alamos National Laboratory report LA-13034MS (1996).

8. R. O. Elliot, K. A. Gschneidner, and C. P. Kempter, "Thermal Expansion of some Delta Plutonium Solid Solution Alloys" in PLUTONIUM 1960 (Cleaver-Hume Press Ltd., London).

9. R. L. Moment, J. Cryst. Grwth. 2 (1968) 15.

10. H. M. Ledbetter and R. L. Moment, Acta Metall. 24 (1976) 891.

11. F. W. Schonfeld, E. M. Cramer, W. N. Miner, F. H. Ellinger, and A. S. Coffinberry, "Plutonium Constitutional Diagrams" in Prog. in Nucl. Energy, Series V, Vol. 2Metallurgy and Fuels (Pergamon Press, New York, 1959) 579.

12. R. L. Moment, "Annealing and Deformation Twinning in Delta-Stabilized Plutonium-Gallium Alloys" in PLUTONIUM 1970 (The Metallurgical Society of the American Institute of Mining, Metallurgical, and Petroleum Engineers, Inc., New York). 
THE CRITICAL RECRYSTALLIZATION STRAIN AND GRAIN GROWTH OF GALLIUM-STABILIZED $\delta$-PLUTONIUM

We are suspended in language in such a way that we cannot say what is up and what is down.

$-\mathrm{N}$. Bohr

\author{
6.1 ABSTRACT \\ 6.2 INTRODUCTION \\ 6.3 EXPERIMENTAL PROCEDURES \\ 6.3.1 Alloy Preparation \\ 6.3.2 Homogenization and Metallography \\ 6.3.3 Strain \\ 6.4 RESULTS \& DISCUSSION \\ 6.4.1 Chemical Purity \\ 6.4.2 Effect of Strain State and Magnitude \\ 6.5 CONCLUSION \\ ACKNOWLEDGMENTS \\ REFERENCES
}

\title{
6.1 ABSTRACT
}

The current chapter reports on the growth of small single crystals of fcc $(\delta)$-plutonium ( $1 \mathrm{wt} \%$ gallium alloy) by a strain-annealing method. In the $1 \mathrm{wt} \%$ gallium alloy, solidstate strain anneal is well suited to grow crystals, in order to avoid the sequence of phase transformations that occur on cooling from the liquid. Because plutonium is a toxic and fissile metal, it was necessary to adopt a punch stretching technique that could be carried 
out in a glovebox to allow one to obtain the critical conditions for grain growth without deforming an excessive number of samples. Toward this end, three polycrystalline disks of the $\delta$-plutonium $1 \mathrm{wt} \%$ gallium alloy were plastically deformed at room temperature with a biaxial punch. After a $90 \mathrm{hr}$ isothermal anneal at $480^{\circ} \mathrm{C}$ in an argon atmosphere, grains with a volume of $1 \mathrm{~mm}^{3}$ were produced. Post-anneal analysis of the disk surface, at the location of the largest grain, gives the following values for the true (logarithmic) principal strain: radial strain $\left(\varepsilon_{1}=0.02\right)$, circumferential strain $\left(\varepsilon_{2}=0.02\right)$, and throughthickness strain $\left(\varepsilon_{3}=-0.04\right)$. These critical strain values compare favorably with published literature data obtained using deformation paths other than biaxial deformation.

\subsection{INTRODUCTION}

Pure plutonium melts at $641^{\circ} \mathrm{C}$ and exhibits six allotropes between room temperature and the liquid phase. One phase of interest, the fcc $\delta$-phase, is thermodynamically stable over the temperature range $319^{\circ} \mathrm{C}$ to $451^{\circ} \mathrm{C}$. However, small additions of gallium help retain the $\delta$-phase to room temperature $[1,2]$. The appropriate portion of the plutoniumgallium binary phase diagram [3] near the $1 \mathrm{wt} \%$ gallium composition is shown in Figure 6.1. The advantage of using the $1 \mathrm{wt} \%$ gallium alloy is that the number of solidstate transformations is reduced from six to two. Even with the gallium addition, this alloy experiences a phase transformation while cooling to room temperature. Consequently, this phase transformation makes crystal growth using classical solidification techniques extremely difficult. Therefore, we elected to adopt a solid-state strain-anneal technique. 


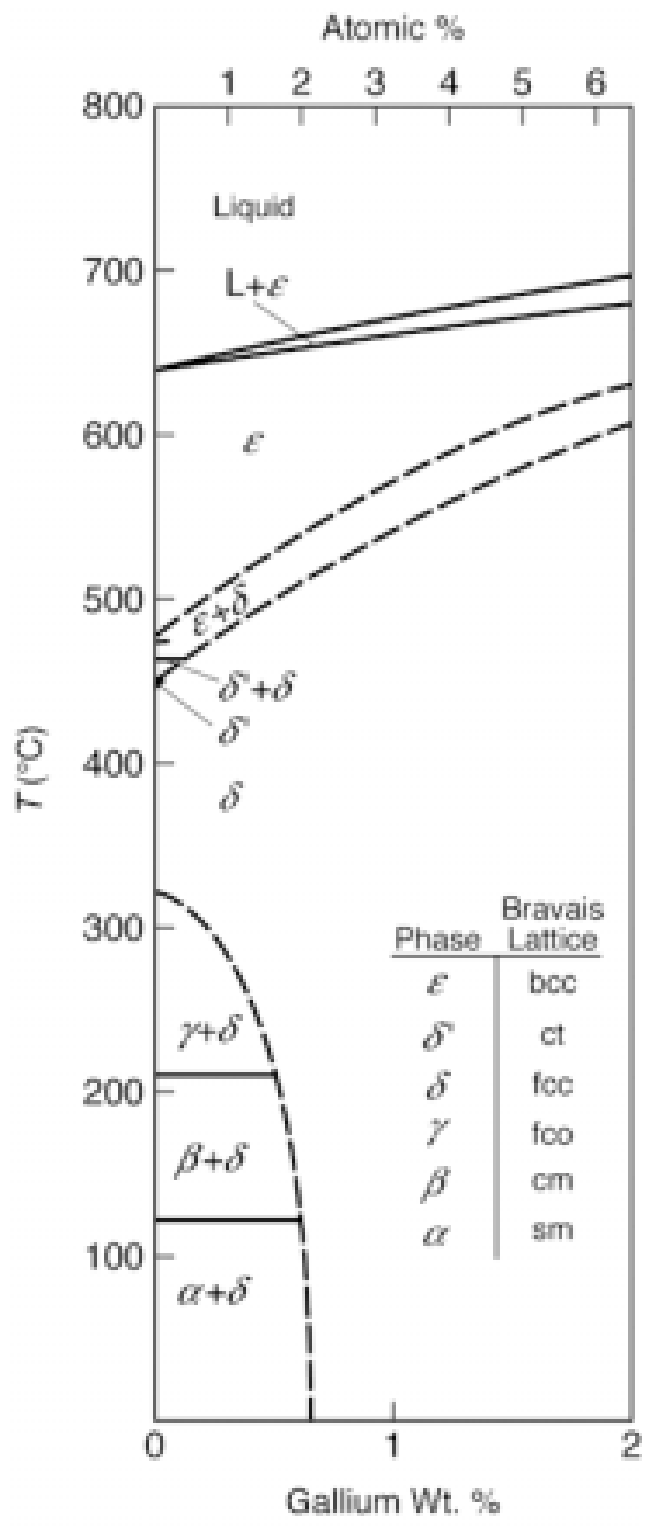

Figure 6.1. The low gallium composition of the plutonium-gallium binary phase diagram. Pure plutonium melts at $641^{\circ} \mathrm{C}$ and transforms from the $\varepsilon$-phase to the $\delta$-phase, and so on through $\delta, \gamma, \beta$, and finally the room temperature monoclinic $\alpha$-phase. In the $1 \mathrm{wt} \%$ gallium alloy, the number of solid-state transformations are reduced from six to two. The respective Bravais lattices for each of the thermal allotropes of plutonium are shown in order to illustrate the complex phase behavior.

The strain-anneal technique is based upon the recrystallization of deformed materials where new grains of the stable phase grow at the expense of the deformed grains [4]. When a metal is plastically deformed, a small portion of the deformation energy is stored 
in the lattice as dislocations, while the majority of energy is dissipated as heat [5]. The stored dislocations provide the driving force for the recrystallization and grain growth processes. The minimum strain that produces the largest grains upon recrystallization defines a critical plastic strain value. At this value, the dislocation density makes it statistically favorable to form only a few nuclei [6]. These nuclei can then grow at the expense of the unrecrystallized material. At deformations less than $10 \%$, the critical strain for recrystallization and grain growth exhibits a strong dependence on the annealing temperature [7]. This temperature dependence is only known imprecisely for small amounts of plastic strain for plutonium-gallium alloys, and in order to revisit this problem we found it necessary to fix the annealing temperature and empirically determine the critical plastic strain at that temperature.

Beer and Pavlick [7] found that very precise control of both strain and temperature was needed to obtain the critical condition for grain growth. They deformed tensile specimens to different strain amount, in increments of $1 \%$ strain, and found that the window width for grain growth was only $2 \%$ strain at a given annealing temperature. This creates a dilemma: how does one obtain the critical conditions for grain growth reproducibly without deforming an excessive number of specimens? The hazardous properties of plutonium preclude the possibility of deforming an excessive number of specimens in a glovebox. Therefore, one must be able to deform samples in a glovebox while reducing the amount of plutonium. The researchers who have been successful applying the strain-anneal grain growth technique to plutonium have addressed this issue by introducing a strain gradient in their specimen or a temperature gradient in their 
furnace [8-10]. Thus, the optimal combination of strain and temperature is present in part of the sample.

In all of the successful experiments described in the literature the stress-state was uniaxial tension or compression. Moment [9] deformed a square bar by bending it around a mandrel producing a gradient in both tensile and compressive deformation on either side of the bar. Berndt [8] pulled a tapered tensile sample to fracture, producing a deformation gradient. Finally, Nurminen [10] deformed tensile specimens uniformly, but then performed a temperature gradient anneal.

In the current work a deformation gradient was established in our specimen, which was then subjected to an isothermal anneal. Disks of material were deformed using biaxial punch stretching to different dome heights. The deformation gradient was found by deforming commercial purity aluminum sheets in which the punch stretching produced a deformation gradient giving a $15 \%$ strain difference within a single specimen. This difference was determined through circle grid measurements. An aluminum surrogate was chosen because electroplating a circle grid pattern on plutonium is not possible. The punch-stretched disks were $25.4 \mathrm{~mm}$ in diameter resulting in a shallow strain gradient despite the $15 \%$ strain difference. Thus, this particular deformation technique results in a large volume of material at a particular strain, producing ample material at the critical strain in which large grains could grow.

In our punch testing experiment the stress-state varies within the stretched disk from plane strain deformation at the perimeter to balanced biaxial stress in the center of the disk. Thus, the deformation state is not as simple to analyze as in the case of uniaxial tension. However, by using an effective measure of strain, we were able to evaluate the 
deformation state and compare critical-strain values from our experiments to the previous published results. This approach to measuring the critical strain for recrystallization depends on measuring the position of the largest grains with respect to the disk perimeter/pole, knowing the dome height of the disk, and having a series of circle-gridded calibration samples.

\subsection{EXPERIMENTAL PROCEDURE}

\subsubsection{Alloy Preparation}

The starting material for this study was double electrorefined (ER) plutonium that had been vacuum cast into rods having a total impurity level of $450 \mathrm{ppm}$ by weight. The elemental impurities were measured by inductively coupled plasma (ICP) mass spectroscopy. This technique analyzes 75 elements in total. The significant metallurgical impurities along with the detection limits and relative standard deviation for each of the major impurity elements are listed in Table 6.1. The metal, totaling approximately $200 \mathrm{~g}$, was cut into short lengths and inserted into an electromagnetic levitation crucible along with the quantity of gallium required to make a $1.0 \mathrm{wt} \%$ gallium alloy. This charge was induction melted at $800^{\circ} \mathrm{C}$ using induction coils with a variable frequency $4 \mathrm{kHz}$ operated at $40 \mathrm{~kW}$ of power. The plutonium-gallium melt was levitated for $45 \mathrm{~min}$ at $10^{-5} \mathrm{~Pa}$ to ensure thorough mixing by the induction currents, while distilling away volatile impurities. A complete description of this process has been published previously [11].

\subsubsection{Homogenization and Metallography}

The $\delta$-Pu ingot had an as-cast density of $15.938 \mathrm{~g} \mathrm{~cm}^{-3}\left( \pm 0.002 \mathrm{~g} \mathrm{~cm}^{-3}\right)$ and an average grain size of $15 \mu \mathrm{m}$. This compares to the theoretical density for the $\mathrm{Pu} 1 \mathrm{wt} \%$ gallium alloy of $15.80 \mathrm{~g} \mathrm{~cm}^{-3}$ for a fully homogenized sample. Thus, a small amount of 
Table 6.1. Metallurgically significant impurities. Analysis of this material was performed using ICP-MS, and a total of 75 elements are detected. This analysis excludes the elements $\mathrm{H}, \mathrm{N}, \mathrm{O}, \mathrm{F}, \mathrm{Cl}, \mathrm{Br}$, and I.

\begin{tabular}{|c|c|c|}
\hline Impurity Element & $\begin{array}{l}\text { Concentration } \\
\left(\mathrm{ppm}\left[\mu \mathrm{g} \mathrm{g}^{-1}\right]\right)\end{array}$ & $\begin{array}{c}\text { Instrument Detection Limit } \\
\left(\mathbf{p p m}\left[\mu \mathrm{g} \mathrm{g}^{-1}\right]\right)\end{array}$ \\
\hline Sodium & $<8.80$ & 8.80 \\
\hline Aluminum & $<5.60$ & 5.60 \\
\hline Silicon & 1.00 & 0.32 \\
\hline Phosphorus & 7.00 & 5.60 \\
\hline Potassium & 40.00 & 14.00 \\
\hline Iron & $<60.00$ & 60.00 \\
\hline Nickel & $<0.40$ & 0.40 \\
\hline Tungsten & 10.00 & 2.20 \\
\hline Uranium $^{1}$ & 121.0 & 0.07 \\
\hline Americium $^{2}$ & 1.50 & 0.45 \\
\hline
\end{tabular}

${ }^{1}$ The ${ }^{235} \mathrm{U}$ isotope results from the $\alpha$-decay of the ${ }^{239} \mathrm{Pu}$ nuclei, growing in at a rate of approximately $30 \mathrm{ppm}$ per year.

${ }^{2}$ The ${ }^{241} \mathrm{Am}$ isotope results from the $\beta$-decay of the ${ }^{241} \mathrm{Pu}$ nuclei. Americium grows in at a rate of approximately $120 \mathrm{ppm}$ per year, depending on the initial ${ }^{241} \mathrm{Pu}$ concentration. These daughter products are always present as impurities in Pu metal.

the higher density $\delta$-phase is retained in areas of the sample that are gallium deficient. The inhomogeneous gallium concentration was removed with a homogenization treatment at $440^{\circ} \mathrm{C}$ for $50 \mathrm{hrs}$. The homogenization process is an isothermal anneal step where the temperature is held within the upper region of the stable $\delta$-Pu phase field to increase the rate of gallium diffusion. After the ingot had been homogenized, it exhibited an average grain size of $30 \mu \mathrm{m}$, and the grain shape was equiaxed. Specimens for subsequent crystal growth experiments were cut from the ingot using a diamond saw with Freon coolant. The disks were mechanically polished inside an inert atmosphere glovebox on polishing wheels starting at 230 -grit and ending up with $0.25 \mu \mathrm{m}$ diamond. 
The disks were electrochemically etched with a high current in a solution of $10 \%$ nitric acid in dimethylformamide to delineate the grain boundaries, and the grains were photographed metallographically at different magnifications. The density was determined by the float-immersion method in a bath of bromobenzene.

\subsubsection{Strain}

Our punch stretching technique involves stretching a thin sheet of metal over an unlubricated hemispherical punch as shown in Figure 6.2. The edges of the sheet are rigidly clamped, and the deformation is done at room temperature. In order to determine the strain that was applied to the $\delta$-Pu disks, a calibration curve was constructed, which correlates the strain present in the disk to its dome height. The calibration was done using a series of five 1100-grade $\mathrm{Al}$ disks. Aluminum was chosen because of the similarity of its yield and work-hardening behaviour to that of the $\mathrm{Pu}-1 \mathrm{wt} \% \mathrm{Ga}$ alloy. Aluminum disks were deformed at a punch displacement rate of $0.50 \mathrm{~mm} \mathrm{~min}^{-1}$ at ambient tempera-

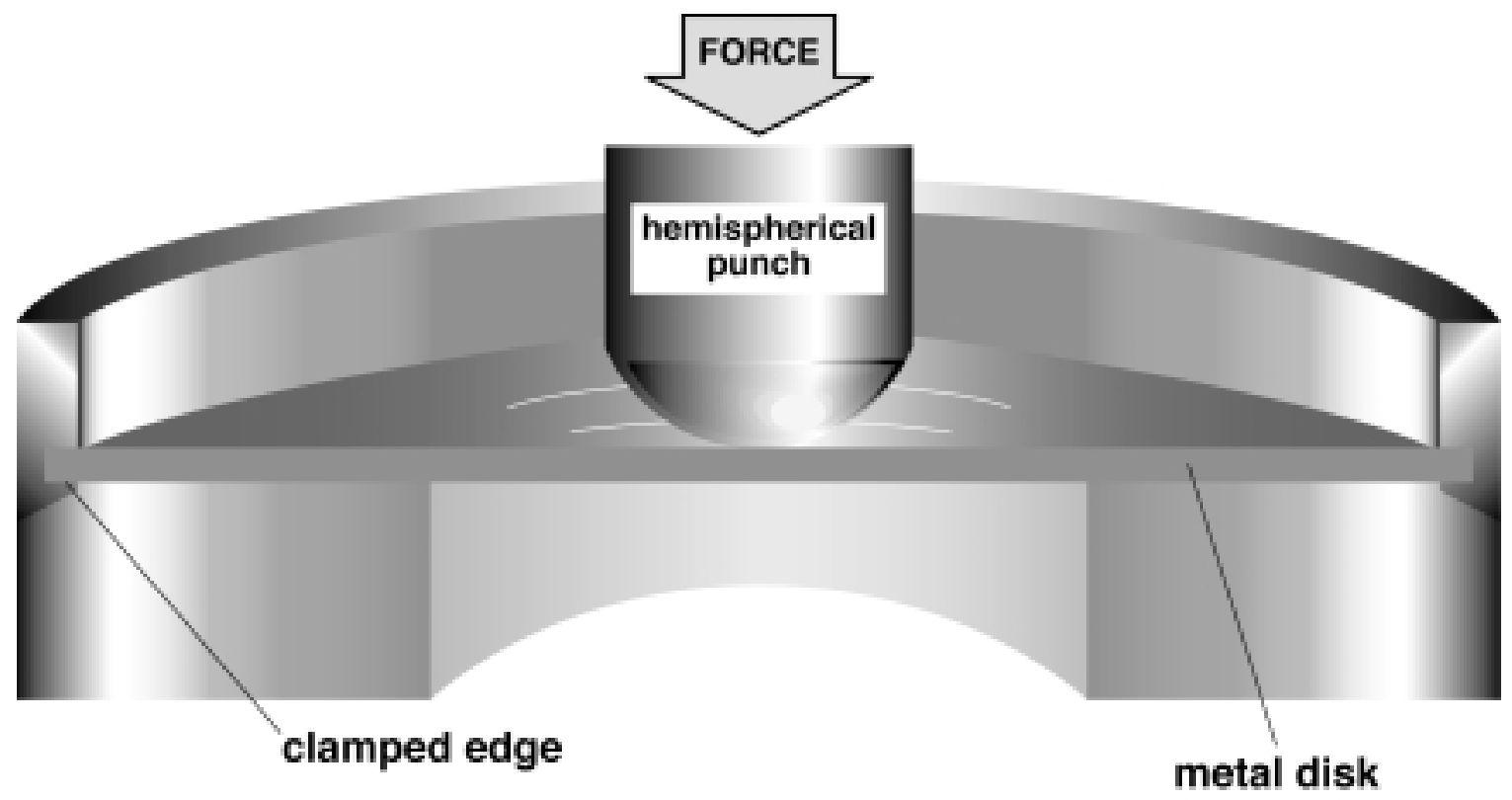

Figure 6.2. A schematic of our punch-stretching experiment that was used to prepare specimens for strain annealing. 
ture and pressure. These disks were the same size as their plutonium counterparts. Prior to testing the $\mathrm{Al}$ disks, we electrochemically etched circular grids onto the surface to allow a direct measurement of strain as a function of dome height. A photograph of a deformed $\mathrm{Al}$ disk is shown in Figure 6.3. The dimensions of circles along two orthogonal lines were measured prior to mounting the undeformed disks in the biaxial die as previously described in the literature [12]. Each disk was aligned such that a circle was directly at the pole. After stretching, the dimensions of the same circles were measured. The differences of the circle dimensions before and after stretching were converted to a true (logarithmic) strain measure in a polar coordinate reference frame, $\varepsilon_{\text {radial }}=\varepsilon_{1}$ and $\varepsilon_{\text {circumferential }}=\varepsilon_{2}$. From constancy of volume, $\varepsilon_{1}+\varepsilon_{2}+\varepsilon_{3}=0$, we obtained the through-thickness strain: $\varepsilon_{\text {through-thickness }}=\varepsilon_{3}=-\left(\varepsilon_{1}+\varepsilon_{2}\right)$.

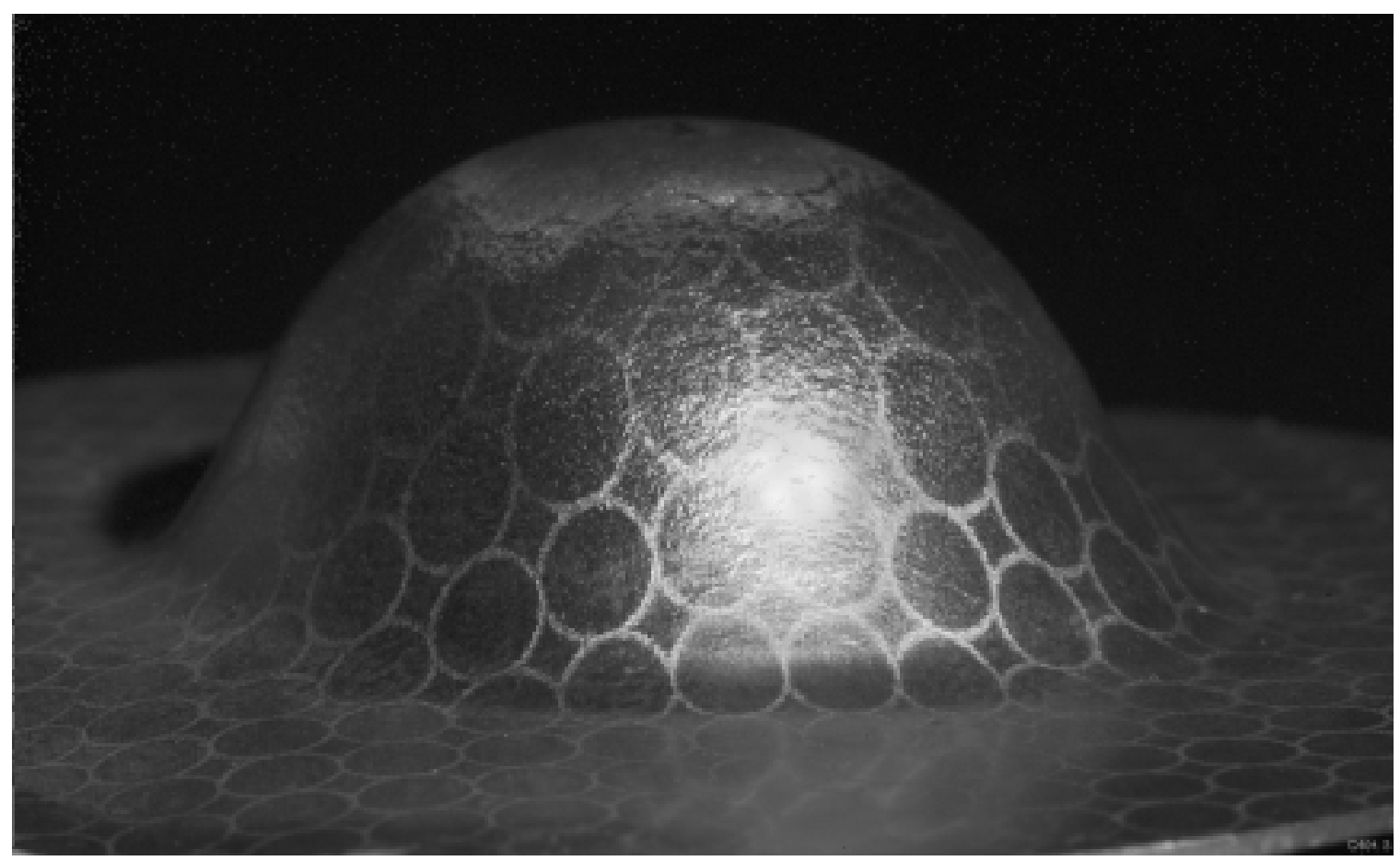

Figure 6.3. The photograph shows an aluminum disk after stretching to a dome height of $11.0 \mathrm{~mm}$. The circle grid pattern was measured before and after stretching to obtain a calibration for in plane strain as a function of dome height. 
In the case of such a punch experiment, stretching deformation with a clamped edge, the stress state varies between biaxial stress at the pole to plane-strain deformation at the outer circumference. ${ }^{2}$ In order to compare the deformation over the entire disk (waist to pole), a measure of effective strain must be used. For this purpose we selected the von Mises effective strain, $\varepsilon_{\text {von Mises }}$, which can be written in terms of the true principal strains measured with the circle grid:

$$
\varepsilon_{\text {von Mises }}=\frac{\sqrt{2}}{3}\left(\sqrt{\left(\varepsilon_{1}-\varepsilon_{2}\right)^{2}\left(\varepsilon_{2}-\varepsilon_{3}\right)^{2}+\left(\varepsilon_{3}-\varepsilon_{1}\right)^{2}}\right)
$$

\subsection{RESULTS AND DISCUSSION}

\subsubsection{Chemical Purity}

The total impurity content of the plutonium is listed in Table 6.1. The impurities of metallurgical importance are $\mathrm{Al}, \mathrm{Si}, \mathrm{Fe}, \mathrm{Ni}, \mathrm{U}$, and $\mathrm{Am}$. Uranium is present at the highest concentration; however, at the $100 \mathrm{ppm}$ level uranium does not form insoluble impurities that could affect grain growth. Iron and nickel both form low-melting eutectics at concentrations greater than $200 \mathrm{ppm}$, but levels are well below this concentration. Thus the iron and nickel present will not form the intermetallic compounds that could affect grain growth. High concentrations of aluminum and silicon can serve to stabilize the $\delta$-phase [1]. In the present case the concentrations are low enough that this will not occur. Based on this analysis we do not consider that the impurities that are present will effect recrystallization and grain growth in our experiments.

\footnotetext{
${ }^{2}$ There is a possible source of experimental error associated with figure 6.2. The error arises from material flow from the edge of the clamp disk. Although we have not measured this error, it is anticipated that the error will be small for small dome height displacements.
} 


\subsubsection{Effect of Strain State and Magnitude}

The strain distributions obtained from an aluminum disk deformed to a dome height of $3.0 \mathrm{~mm}$ are shown in Figure 6.4. In this figure the true principal strain is plotted against position on the disk relative to the pole of the disk. One can see a symmetrical strain distribution about the origin that drops off from the pole as expected. At the pole

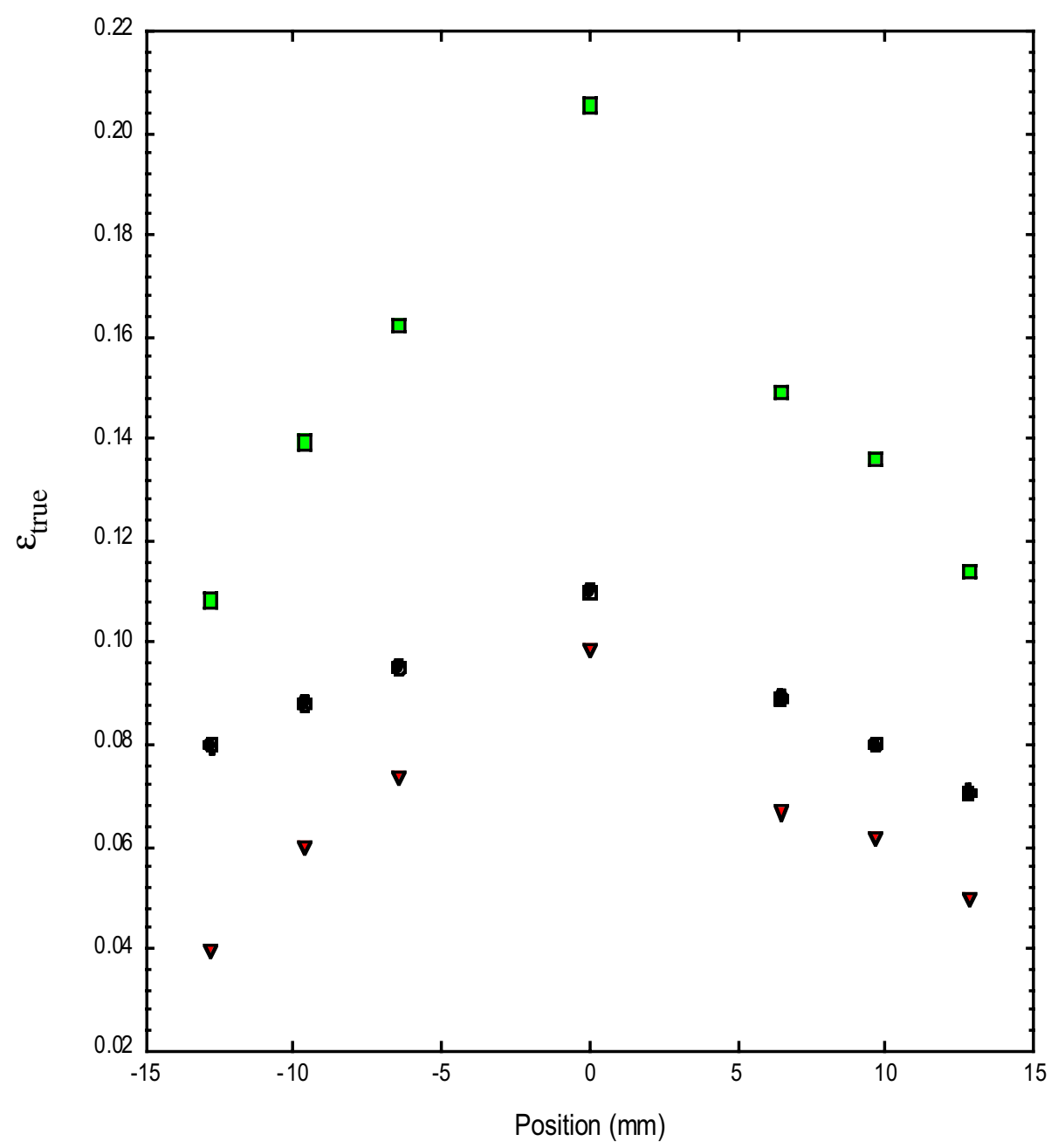

Figure 6.4. Strain calibration from an aluminum disk that was stretched to a dome height of $3.0 \mathrm{~mm}$. In this plot, the true strain: radial (round points), circumferential (triangular points), and through-thickness strain (square points) are plotted against position along the aluminum disk. The through-thickness strain is calculated from the constancy of volume relationship. 
$\varepsilon_{1}=\varepsilon_{2}$, a result of balanced biaxial tension. One can see that the strain state approaches plane strain, $\varepsilon_{2}=0$, at the edge of the disk. To quantify the strain-state throughout the stretched disk, we elected to use the von Mises effective strain measure as expressed in Equation (6.1). The effective strain will be a function of deformation mode and will vary with the strain-state from the outer circumference to the pole of the stretched disk. The effective strain will also be a function of the height to which the disk is deformed. The effective strain value increases with increasing dome height. We deformed disks to dome heights of 2.0, 3.0, 4.0, and $5.0 \mathrm{~mm}$. Thus, we have a separate von Mises strain distribution from waist to pole for each of these conditions. These data, von Mises strain versus distance from the pole of the dome, are shown in Figure 6.5 for each height of dome. One can see that the profiles all have the same shape, irrespective of dome height, and that there is a significant gradient in effective strain for each disk. For example, the disk with a dome height of $2.0 \mathrm{~mm}$ deformed to an effective strain of only 0.025 at the outer circumference, despite having an effective strain of 0.175 at the dome pole. Thus, there is a very large window of effective strain in these samples for the critical conditions for grain growth to occur in a sample.

The literature results for the strain-anneal technique all indicated critical effective strains for recrystallization and grain growth below 0.10 . Based on these data and the effective strain profiles shown in Figure 6.5, we elected to deform our Pu 1wt \% Ga disks to a dome height of $2.0 \mathrm{~mm}$. Deformation to this dome height resulted in a maximum volume of material in a strain condition where the development of a large grain was possible. Subsequent to deformation, the disk was annealed at $480^{\circ} \mathrm{C}$ for $90 \mathrm{hrs}$. The $480^{\circ} \mathrm{C}$ anneal is at higher temperature than that used by Beer and Pavlick [7] and below 


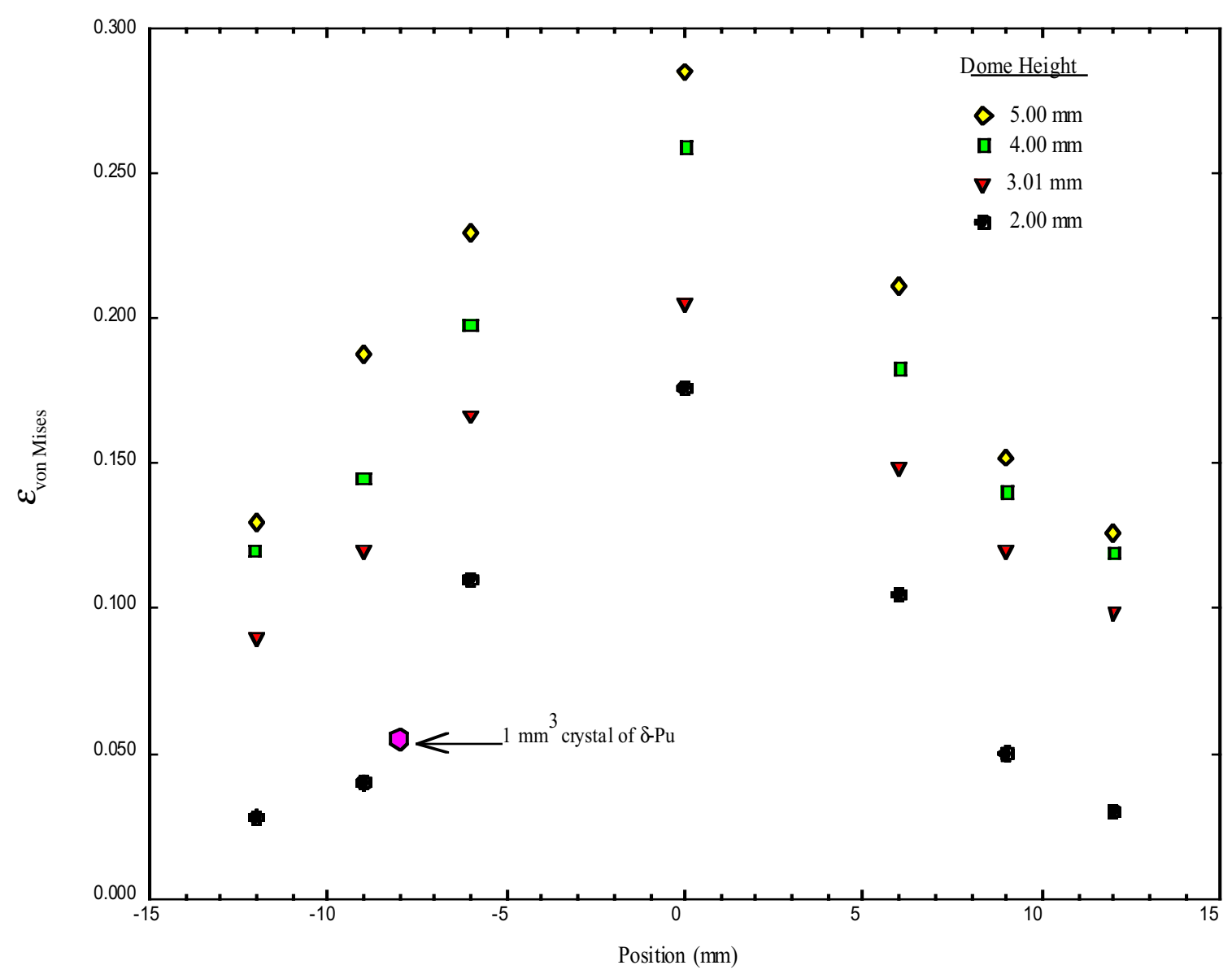

Figure 6.5. The calculated effective strains for aluminum disks that were stretched to different dome heights. The location of the large $\delta$-Pu grain is indicated on the plot.

that of Moment [9]. The surfaces of the deformed plutonium disks were polished to reveal grain boundaries. We found that straining did result in grain growth. The largest grain was about $1 \mathrm{~mm}$ on a side with an approximate volume of $1 \mathrm{~mm}^{3}$. The grain was located $8 \mathrm{~mm}$ from the pole in the disk that was stretched to a height of $2.0 \mathrm{~mm}$. A metallographic image of this grain is shown in Figure 6.6. The grain appears as the darkest area inside the enclosed circle on the photomicrograph. The contrast between grains is a function of the amount of oxidation as it relates to its crystallographic orientation. Thus, the darker grains correspond to the most oxidation. The specimen was 


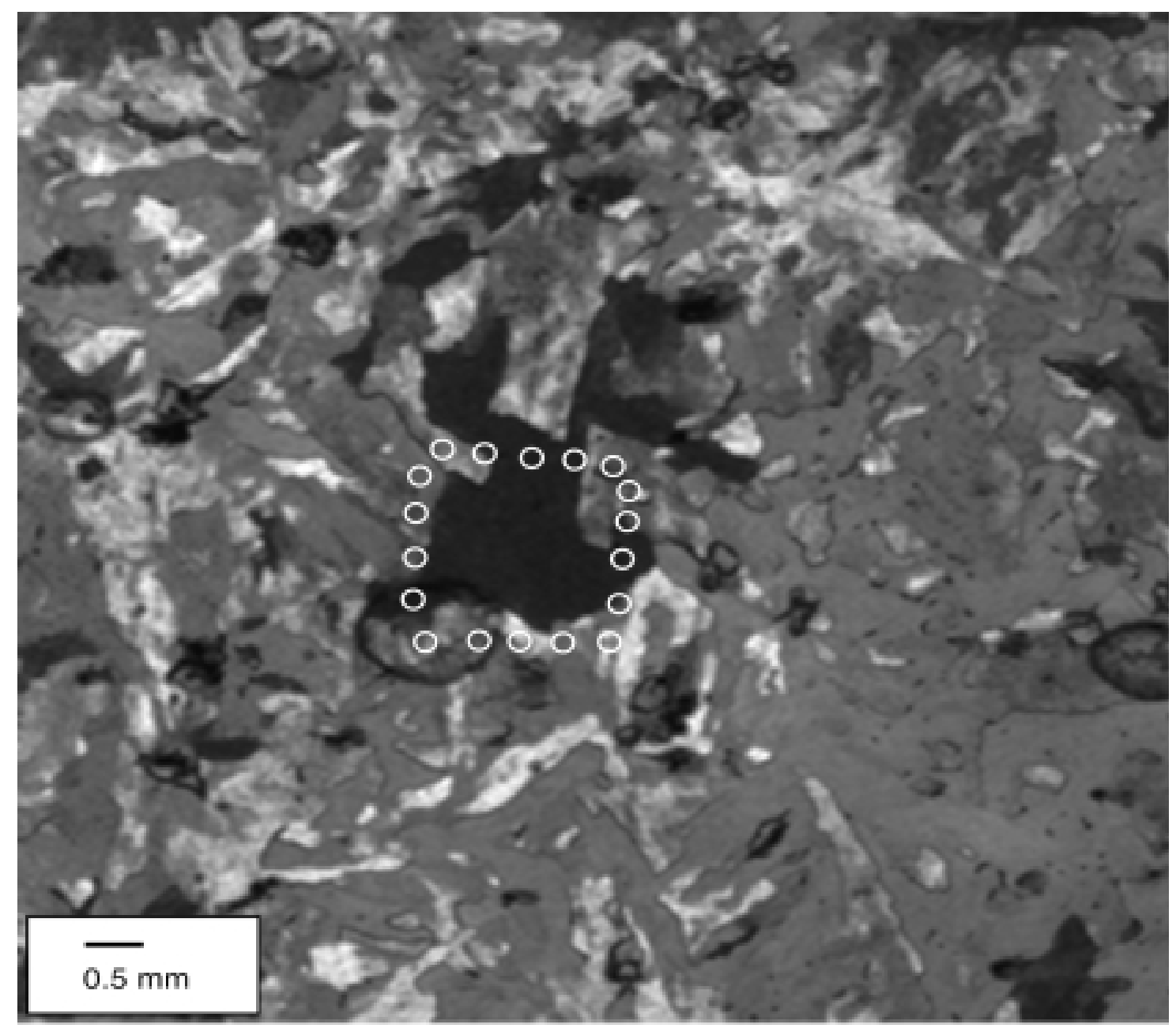

Figure 6.6. A large grain of $\delta$-phase with a volume of $1 \mathrm{~mm}^{3}$ is the dark boundary located inside the circle. The photomicrograph was done on a microscope with reflected light illumination in the polarized position. The contrast between the grains is a function of the amount of oxidation as it relates to its crystal orientation. Darker grains are those that oxidize the fastest. In this location, the values of the strain components obtained from the calibration curve are radial strain $\varepsilon_{1}=0.02$, circumferential strain $\varepsilon_{2}=0.02$, and throughthickness strain $\varepsilon_{3}=-0.04$.

mechanically polished and then electrochemically polished. The grain structure was revealed with a stain-etch procedure. The sample was submerged and etched in a electrochemical solution of $10 \%$ nitric acid in dimethylformamide at 3 volts dc. The image was taken on a microscope with reflected light illumination in the polarized position. From our calibration data, strains/dome height, the large grain experienced a radial strain of 
$\varepsilon_{1}=0.02$, circumferential strain of $\varepsilon_{2}=0.02$, and through-thickness strain of $\varepsilon_{3}=-0.04$. These values of principal strain correspond to a von Mises effective strain value of 0.04 , shown in Figure 6.5. The strain field over our entire large grain is slightly nonuniform and complex. However, we can compute the von Mises effective strain, which accounts for the strain-state under complex loading, to allow a first-order comparison of our critical strain values to the literature.

There have been three previously reported critical plastic strain values for $\delta$-Pu, which are summarized in Table 6.2. It should be noted that each of these investigations involves a uniaxial stress state. Thus, as our deformation state is more complex, we must use the von Mises measure of strain for comparison. Moment [9] bent a $0.53 \mathrm{~cm}$ square cross-section rod around a mandrel to produce a range of strains within the sample.

Table 6.2. Critical strains for strain-anneal grain growth. The critical strain varies with temperature, and all of the reported values are consistent with this trend; however the origin of the temperature dependence may result from the narrow $\delta$-Pu phase field.

\begin{tabular}{|lccccc|}
\hline Researcher & Year & $\begin{array}{c}\text { Deformation } \\
\text { Mode }\end{array}$ & $\begin{array}{c}\text { Stress } \\
\text { State }\end{array}$ & $\begin{array}{c}\text { Annealing } \\
\text { Temperature }\end{array}$ & $\begin{array}{c}\text { Critical } \\
\text { Strain } \\
\text { (von Mises) }\end{array}$ \\
\hline Moment & 1966 & bending & $\begin{array}{c}\text { uniaxial tension } \\
\text { and } \\
\text { compression }\end{array}$ & $500^{\circ} \mathrm{C}$ & 0.02 \\
\hline $\begin{array}{l}\text { Beer and } \\
\text { Pavlick }\end{array}$ & 1967 & $\begin{array}{c}\text { uniaxial } \\
\text { tension }\end{array}$ & $\begin{array}{c}\text { uniaxial } \\
\text { tension }\end{array}$ & $\begin{array}{c}375^{\circ} \mathrm{C} \text { and } \\
450^{\circ} \mathrm{C}\end{array}$ & 0.06 to 0.08 \\
\hline Nurminen & 1969 & $\begin{array}{c}\text { uniaxial } \\
\text { tension }\end{array}$ & $\begin{array}{c}\text { uniaxial tension } \\
\text { unknown, } \\
\text { gradient } \\
\text { furnace }\end{array}$ & 0.0175 \\
\hline Current Study & 2000 & punch & biaxial tension \\
stretching & $480^{\circ} \mathrm{C}$ & 0.04 \\
& \multicolumn{5}{c}{$\begin{array}{c}\text { to } \\
\text { plane strain } \\
\text { deformation }\end{array}$} \\
\hline
\end{tabular}


In this case the maximum principal strain, which we will compare to our von Mises strain, can be estimated from the simple beam equation:

$$
\varepsilon_{1}=\frac{y}{\left(R_{0}+0.50 Y\right)} \text {. }
$$

In Equation (6.2) $\varepsilon_{1}$ is the maximum principal strain, $\mathrm{y}$ is the distance from the center of the sample, $Y$ is the height of the sample, and $R_{O}$ is the radius of the mandrel. This equation assumes that the neutral axis remains at the center of the beam's cross section. In other words the compressive and tensile distribution of strains are symmetric. One should note that for this deformation the maximum principal strain and von Mises effective strain are equivalent. The largest grain in this test experienced an effective strain prior to annealing of $\varepsilon_{1}=0.02$. Moment performed his strain-anneal at $500^{\circ} \mathrm{C}$ for six days (144 hours).

Beer and Pavlick [7] performed a second series of strain-anneal experiments. They first rolled and recrystallized sheets of electrorefined $\mathrm{Pu} 1 \% \mathrm{Ga}$ and then strained tensile specimens in increments of $1 \%$ strain, between 0 and $12 \%$ elongation. Following elongation they annealed the tensile samples at both $375^{\circ} \mathrm{C}$ and $450^{\circ} \mathrm{C}$ for 24 hrs. Beer and Pavlick did not observe any grain growth for the specimens strained below $6 \%$. They observed recrystallized grains in the samples strained to 0.10 and 0.12 , but the grain size was very fine, only slightly larger than the initial grain size. They did, however, observe recrystallization and accentuated grain growth for the samples deformed to $6 \%, 7 \%$, and $8 \%$ strain. Based on these observations, they deduced that $6 \%$ strain was near the critical strain for a $450^{\circ} \mathrm{C}$ anneal and $8 \%$ the critical strain when the anneal was at $375^{\circ} \mathrm{C}$. Beer and Pavlick also examined the temperature dependency of the critical strain for recrystal- 
lization. They found that for deformations below $10 \%$ strain, the critical strain for recrystallization and grain growth was an exceptionally strong function of the annealing temperature. This strong temperature sensitivity is important in interpreting the critical strain that we measured in our experiments.

Nurminen [10] performed a strain anneal crystal growth study on a Pu $1.22 \mathrm{wt} \%$ gallium alloy. He used a tensile specimen design as Beer and Pavlick did but performed his anneal in a temperature gradient furnace. Thus, he could not report an exact annealing temperature. Nurminen annealed for $84 \mathrm{hrs}$ in and the highest temperature of the gradient furnace $-550^{\circ} \mathrm{C}$. His results were very similar to those of Moment; he reported a critical tensile strain of $1.75 \%$. One can see that our results for critical strain lie between those reported in previous work. They are perhaps most consistent with Beer and Pavlick's study. Our critical strain, $\varepsilon_{\text {von Mises }} \cong 0.04$, was less than they reported, $\varepsilon=0.06$ to 0.08 , but our annealing temperature was also higher, $480^{\circ} \mathrm{C}$, as compared to $375^{\circ} \mathrm{C}$ and $450^{\circ} \mathrm{C}$.

\subsection{CONCLUSION}

We have grown a single crystal of $\delta$-Pu $1 \mathrm{wt} \%$ Ga alloy approximately $1 \mathrm{~mm}$ on a side after punch-stretching a disk to a dome height of $2.0 \mathrm{~mm}$. Based on our calibration experiments, this grain experienced a critical strain of $\varepsilon_{\text {von Mises }} \cong 0.04$. This result is most consistent with the values for critical strain reported by Beer and Pavlick. It is still unknown why our results and those of Beer and Pavlick indicate a critical strain of between 0.04 and 0.08 , depending on annealing temperature, well above that reported by Moment and later by Nurminen. We believe that the annealing temperature can explain the small 
difference in our result from that of Beer and Pavlick. However, we do not think that the higher annealing temperature alone can explain the very small critical strains reported by Moment and Nurminen.

\section{ACKNOWLEDGMENTS}

I am indebted to Michael Stout and David Embury for many long and instructive sessions. I would also like to thank Michael Blau and Ramino Pereya. We thank Manuel Lovato for assistance with the deformation of the aluminum samples. This work benefited from discussions with Roger Moment, Michael Stevens, Karl Staudhammer and Galen Straub. We also wish to thank John Huang and Barbara Martinez for laboratory assistance. Mick Greenbank is acknowledged for photography support. All work was performed at the Los Alamos National Laboratory under the auspices of the Department of Energy.

\section{REFERENCES}

[1] F. H. Ellinger, C. C. Land, and V. O. Struebing, 1964, J. Nucl. Mater., 12, 226 (1967).

[2] B. Hocheid, J. Bedere, S. Despres, and F. Miard, 1967, in Plutonium 1965, edited by M. B.Waldron (Chapman Hall, London) (1967).

[3] W. N. Miner, F. H. Ellinger, D. R. O’Boyle, and F. W. Schonfeld, LA-3870, Los Alamos Scientific Laboratory, Los Alamos, New Mexico (1968).

[4] H. Carpentera and C. Elam, 1929, Proc. Roy. Soc., London, 100A, 329 (1921).

[5] F. R. N. Nabarro, Theory of Crystal Dislocations, (Clarendon Press, Oxford) (1967). 
[6] J. G. Byrne, 1965, Recovery Recrystallization and Grain Growth, The Macmillan Series in Materials Science, edited by M. E. Fine, J. Weertman, and J. R. Weertman, (The Macmillan Company, London) (1965).

[7] B. J. Beer and J. E. Pavlick, 1967, J. Nucl. Mater., 23, 236 (1967).

[8] A. F. Berndt, 1966, J. Less Comm. Met., 10, 71 (1966).

[9] R. L. Moment, J. Nucl. Mater., 20, 341 (1966).

[10] J. I. Nurminen, React. Mater., 12, 475 (1969).

[11] J. C. Lashley, M. S. Blau, R. A. Pereyra, and K. P. Staudhammer, J. Nucl. Mater., 274, 315 (1999).

[12] S. S. Hecker, Sc. Am., November (1976). 


\section{Appendix 1 \\ PURIFIED PLUTONIUM REDUCES BACKGROUND INTERFERENCE IN NUCLEAR CROSS-SECTION MEASUREMENTS}

\section{A.1 INTRODUCTION}

A program important for weapons diagnostics is currently under way to measure the ${ }^{239} \mathrm{Pu}(\mathrm{n}, 2 \mathrm{n}){ }^{238} \mathrm{Pu}$ cross section. These cross-section measurements are performed with GEANIE (GErmanium Array for Neutron-Induced Excitations) [1,2] at the Weapons Neutron Research Facility [3] at Los Alamos National Laboratory by detecting gamma rays from the product nuclei. In early work, the relatively intense gamma-ray background from ${ }^{241} \mathrm{Am}$ impurity in plutonium saturated the germanium detectors with background signals. Hence an improved plutonium sample purified of americium was fabricated [4]. This sample improved the ${ }^{239} \mathrm{Pu}(\mathrm{n}, 2 \mathrm{n}){ }^{238} \mathrm{Pu}$ measurement. The same plutonium sample is being used in a new experiment to measure the ${ }^{239} \mathrm{Pu}\left(\mathrm{n}, \mathrm{n}^{\prime} \gamma\right)^{239} \mathrm{Pu}$ cross section-an important measurement for neutron transport calculations.

Plutonium enriched in ${ }^{239} \mathrm{Pu}$ typically contains the isotopic impurities ${ }^{238} \mathrm{Pu},{ }^{240} \mathrm{Pu}$, and ${ }^{241} \mathrm{Pu}$. The ${ }^{241} \mathrm{Pu}$ nuclei decay into ${ }^{241} \mathrm{Am}$ by beta decay, and its concentration typically increases at a rate of 130 parts per million ( $\mathrm{ppm}$ ) per year, depending on the initial ${ }^{241} \mathrm{Pu}$ concentration [5]. The gamma $(\gamma)$ rays emitted in ${ }^{241} \mathrm{Am}$ decay are much more intense than those emitted from the longer-lived isotopes. Thus, the removal of 
${ }^{241}$ Am provides an improved signal-to-background ratio for gamma-ray measurements. The purification technique and some measurements are described below.

\section{A.2 SAMPLE PREPARATION}

The plutonium sample used in the cross-section measurements on GEANIE was highly enriched ${ }^{239} \mathrm{Pu}$. This sample was processed through a molten-salt-extraction process followed by two electrorefining steps. The sample was then further purified through an electromagnetic-levitation distillation process. In this process, externally applied radio-frequency power operated at $4 \mathrm{kHz}$ and $40 \mathrm{~kW}$ induces an electric current in the levitation crucible, which acts as a transformer inducing a current in the plutonium in the direction opposite to the current flowing in the crucible. An electromagnetic field is created, and the plutonium sample in the crucible is repelled and levitated a small distance ( 2 to $3 \mathrm{~mm}$ ) from the walls of the crucible. Approximately $200 \mathrm{~g}$ of material is processed in a single run. Because americium exhibits a high vapor pressure relative to plutonium, ${ }^{241} \mathrm{Am}$ impurities, amounts typically greater than $100 \mathrm{ppm}$, are distilled away from the plutonium while in the melt and collected onto a water-cooled condenser. The material is distilled for 0.5 to $1 \mathrm{~h}$. Enough gallium was added $(1.0 \mathrm{wt} \%)$ to the plutonium during this purification process to stabilize the sample at the face-centered-cubic $\delta$-phase upon solidification. As the power is reduced, the plutonium is chill-cast directly from the electromagnetic field into the crucible as shown in Figure A.1.

A subsequent heat-treatment step produces a homogenous concentration of gallium throughout the plutonium sample. Gallium-stabilized $\delta$-plutonium can be rolled to produce thin samples of uniform thickness. Thin $(0.25$ and $0.5 \mathrm{~mm})$ sections (disks) of the 
a) $\mathrm{Pu}+$

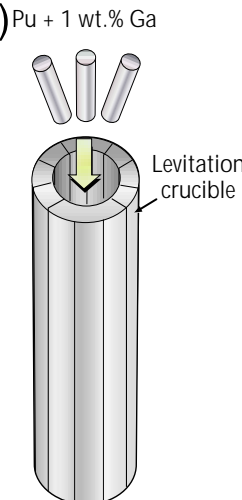

b)
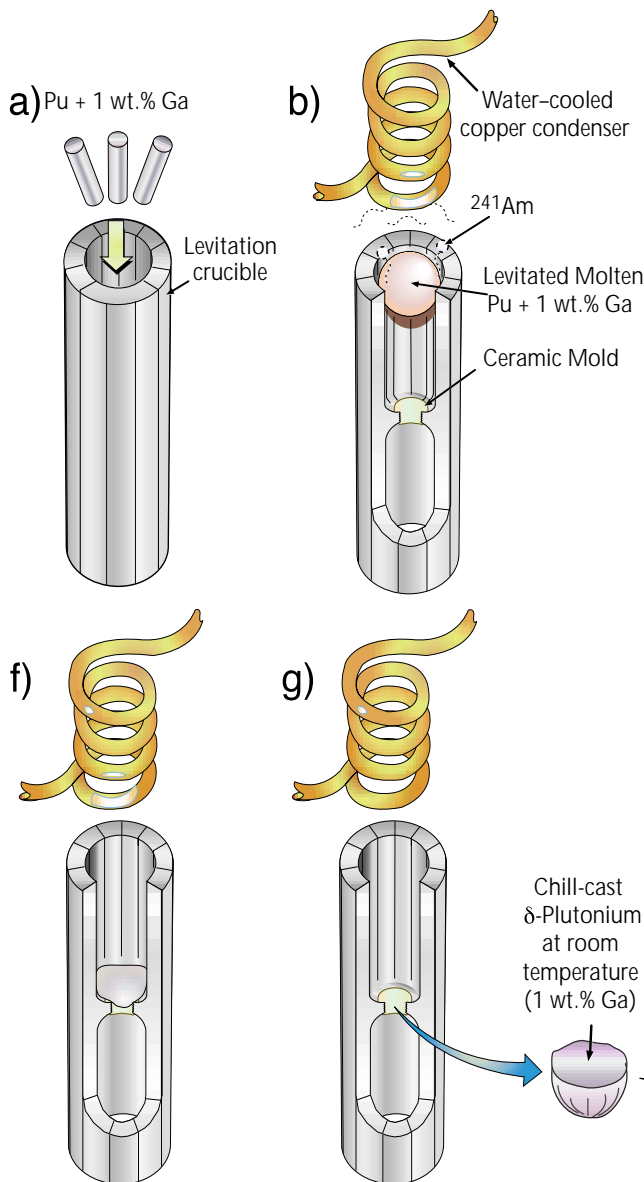

g)

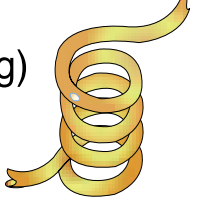

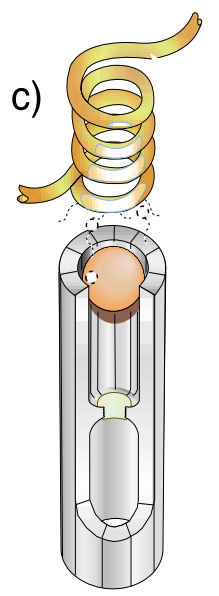
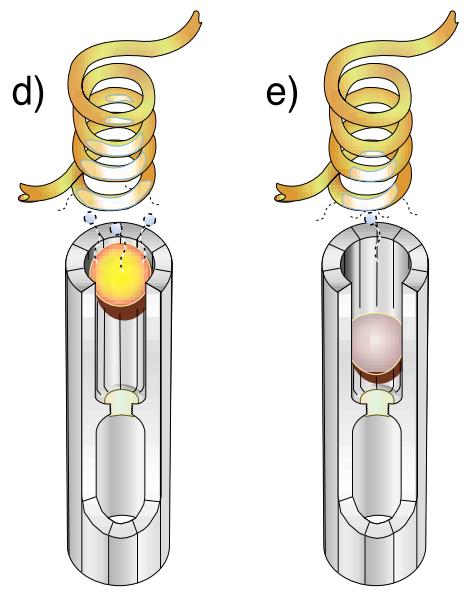

e)

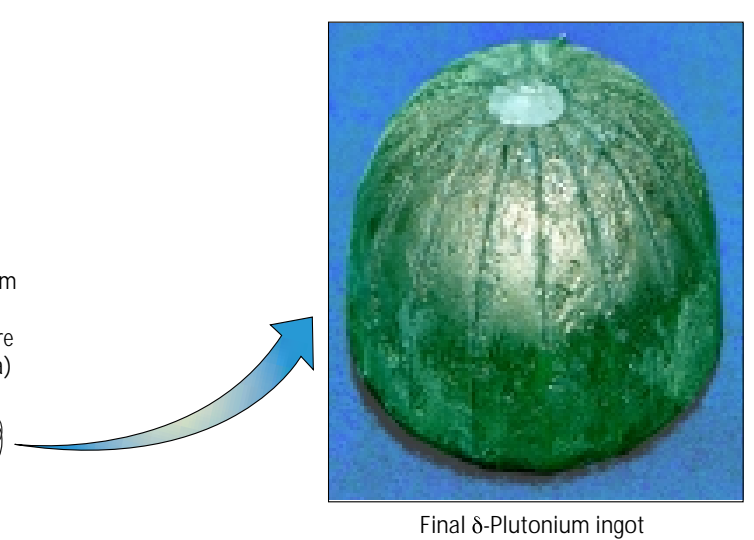

Final $\delta$-Plutonium ingot

Figure A.1. Electrorefined plutonium is placed inside the levitation crucible along with sufficient gallium to stabilize the face-centered-cubic phase upon solidification (a). The mixture begins to melt and levitate a small distance from the crucible walls $(b)$. The gallium begins to mix as the molten-charge plutonium and gallium is levitated, while at the same time volatile impurities like ${ }^{241} \mathrm{Am}$ begin to distill away (c). The charge is allowed to remain in the melt to ensure uniform mixing $(d)$. The furnace power is reduced, and the melt begins to solidify (e). While solidifying, the sample falls directly into a cold ceramic mold (the cooling rates are typically about $373 \mathrm{~K}$ per second) $(f)$. An ingot of $\delta$-plutonium solidifies to conform to the shape of the crucible $(\mathrm{g})$.

purified plutonium sample were thus prepared and encapsulated for safe handling at

GEANIE. A small, thin sample is essential for the success of the gamma-ray technique used. In addition, the material used was selected for high ${ }^{239} \mathrm{Pu}$ enrichment and low ${ }^{241} \mathrm{Pu}$ content. Assays before and after the purification of the plutonium showed that the ${ }^{241} \mathrm{Am}$ content was reduced by a factor of 200 . The final ${ }^{241} \mathrm{Am}$ content was $1.5 \mathrm{ppm}$. 


\section{A.3 NUCLEAR CROSS-SECTION MEASUREMENTS}

The ${ }^{239} \mathrm{Pu}(\mathrm{n}, 2 \mathrm{n}){ }^{238} \mathrm{Pu}$ cross section is important for weapons diagnostics, but knowledge of this cross section as a function of neutron energy is poor. A joint Los Alamos National Laboratory and Lawrence Livermore National Laboratory effort was initiated to measure this cross section using an indirect technique to determine partial $\gamma$-ray cross sections. To deduce the $(n, 2 n)$ reaction-channel cross section, we used nuclear-reaction modeling to determine that portion of the cross section (weak $\gamma$-rays and internally converted $\gamma$-rays) that is not measured with our technique. The accuracy of the technique in part is dependent upon observing $\gamma$-ray transitions between low-lying states in the product nucleus.

We use an array of 26 high-resolution germanium $\gamma$-ray detectors with backgroundsuppression shields (GEANIE) in combination with the high-energy neutron beams from the Weapons Neutron Research (WNR) facility to measure nuclear-reaction cross sections. The excellent energy resolution of the GEANIE detectors allows us to identify specific reaction products from the characteristic $\gamma$-ray energies of low-lying nuclear excited states. The WNR neutron source provides a broad spectrum of incident neutron energies. We use time-of-flight techniques to determine the incident neutron energy for any particular $\gamma$-ray observed. This information gives us the probability or "cross section" for a specific nuclear reaction as a function of the incident neutron energy. Knowledge of these cross sections is useful in applications such as the calculation of integral quantities from microscopic modeling.

In these measurements, we want $\gamma$-rays from reactions on ${ }^{239} \mathrm{Pu}$ to be the dominant signal; therefore, the enrichment in ${ }^{239} \mathrm{Pu}$ must be as high as possible. Our first measure- 
ments using a plutonium sample enriched to $94 \%$ in ${ }^{239} \mathrm{Pu}$ indicated that the $4^{+}$to $2^{+}$ transition at $102 \mathrm{keV}$ was obscured by a peak from the decay of ${ }^{241} \mathrm{Am}$ in the sample. Removal of the ${ }^{241} \mathrm{Am}$ greatly reduced the background interference in the GEANIE detectors shown in Figure A.2. But despite the reduction in background interference from ${ }^{241} \mathrm{Am}$, a strong contaminant line from fission still obscured the $4^{+}$to $2^{+}$transition that we had hoped to observe. We are preparing a report on the results of this work [6].

The use of the particular plutonium selected for recycling and purification also improved the measurement through a reduction in the amount of ${ }^{240} \mathrm{Pu}$ and ${ }^{241} \mathrm{Pu}$ in the material. ${ }^{240} \mathrm{Pu}(\mathrm{n}, 3 \mathrm{n}){ }^{238} \mathrm{Pu}$ contributes background interference to the measurement at

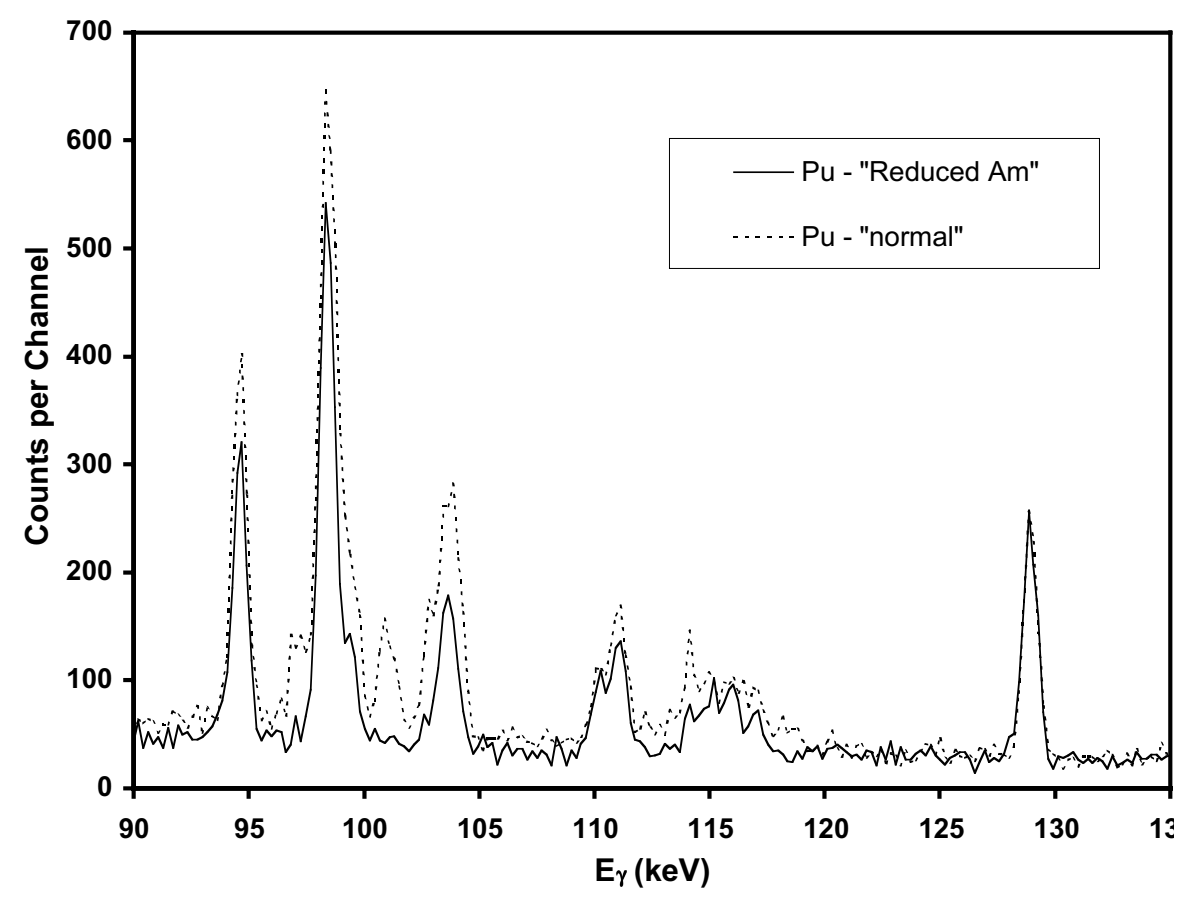

Figure A.2. The plutonium sample background (i.e., no incident neutron beam) photon spectrum in the region of some of the plutonium and uranium K-shell x-rays (94 to 104 $\mathrm{keV}$ ) is shown. The data were taken under identical conditions and normalized to match the "live time," which accounts for the acceptance of the data-acquisition electronics. A $\gamma$-ray from the decay of ${ }^{239} P u$ is visible near $E_{\gamma}=129 \mathrm{keV}$. A reduction in background near $E_{\gamma}=101.8 \mathrm{keV}$ was desirable for the $P u(n, 2 n)$ cross-section measurements and was achieved by reducing the ${ }^{241} \mathrm{Am}$ content of the plutonium sample. 
incident neutron energies greater than $12 \mathrm{MeV}$. Reduced ${ }^{241} \mathrm{Pu}$ content in the sample selected for purification means that ${ }^{241} \mathrm{Am}$ will be less of a problem when the sample is used in future measurements because less americium will grow into the sample.

The inelastic scattering of neutrons from plutonium is also an important quantity for calculations. This quantity, which is poorly known, is needed for neutron transport calculations. We are currently measuring the ${ }^{239} \mathrm{Pu}\left(\mathrm{n}, \mathrm{n}^{\prime} \gamma\right)$ cross section to low-lying states using the same purified plutonium sample used in the ${ }^{239} \mathrm{Pu}(\mathrm{n}, 2 \mathrm{n})^{238} \mathrm{Pu}$ measurement, and we expect to observe thirty $\gamma$-ray lines from neutron inelastic scattering on ${ }^{239} \mathrm{Pu}$. Ten of these $\gamma$-ray lines overlap in energy with $\gamma$-rays from ${ }^{241}$ Am decay. Reducing the ${ }^{241} \mathrm{Am}$ in the plutonium sample is important for us to obtain more accurate inelastic scattering measurements. Figure A.3 shows the decrease in background from the very strong $59.5-\mathrm{keV}$ line from ${ }^{241} \mathrm{Am}$. This greatly reduces the count rate in the detectors while allowing low-energy transitions to be observed more readily.

\section{ACKNOWLEDGMENTS}

The nuclear cross-section measurement was performed by R.O. Nelson, J.A. Becker, and the GEANIE team. This work benefited from contributions made by M. S. Blau, R.A. Pereyra, B. Martinez, and P. Hochanadel. Alan Picklesimer is thanked for first presenting this problem to me. This work was performed under the auspices of the Department of Energy. 


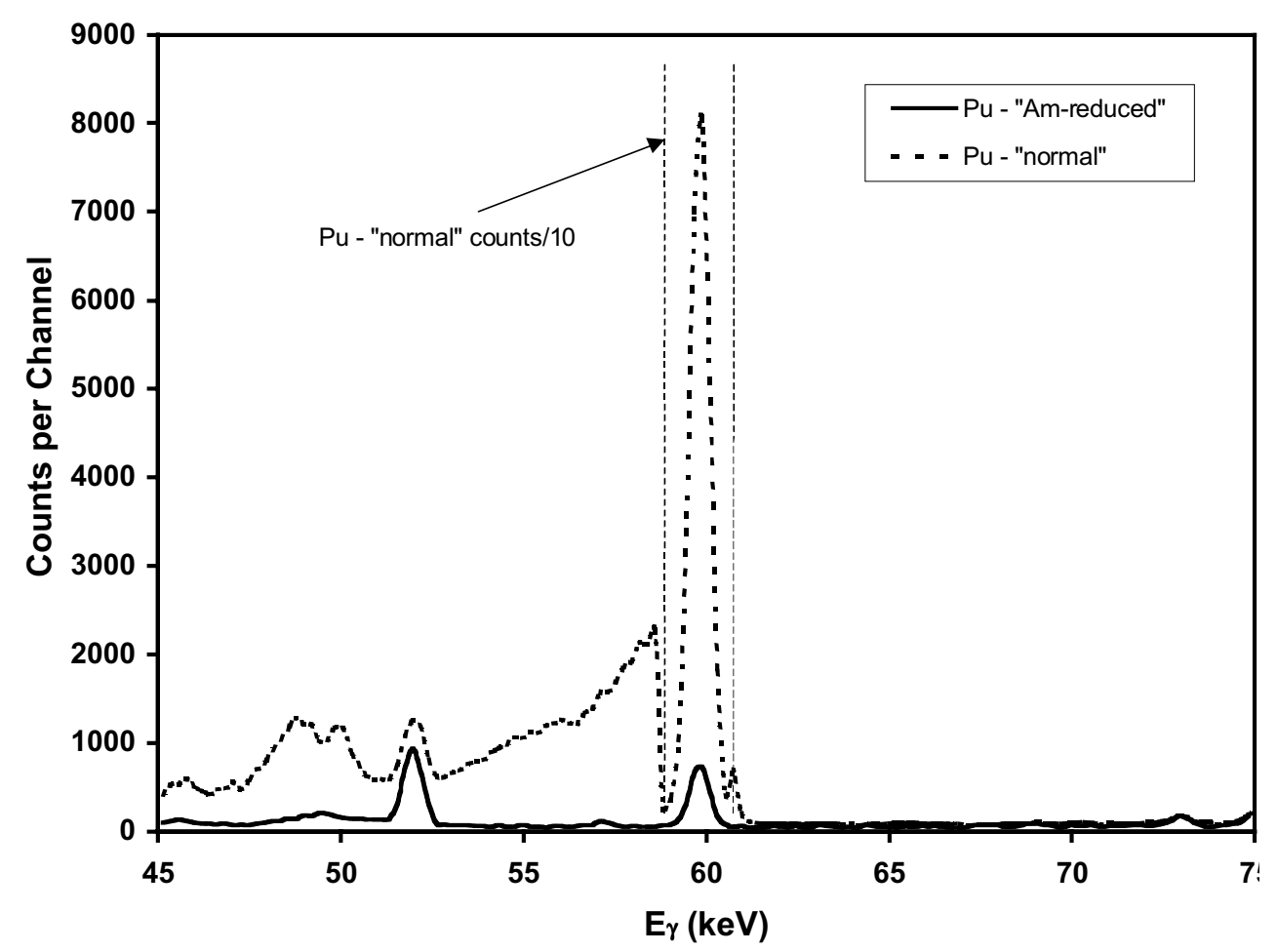

Figure A.3. A background (i.e., no incident neutron beam), low-energy photon spectrum acquired with a "normal" plutonium sample containing ${ }^{241}$ Am "buildup" from the decay of ${ }^{241} \mathrm{Pu}$ is plotted with a spectrum from an "241 Am-reduced" plutonium sample that has undergone molten-salt extraction; zone refining; and the alloying, distillation, and chillcast step. The intense 59.5-keV $\gamma$-ray from ${ }^{241}$ Am decay, which is near the center, demonstrates the huge reduction in counting rate achieved. The peak in the "normal" sample data has been scaled down by a factor of 10 to show the more detailed features in the spectrum. As in Fig. 2, the data were taken under identical conditions and normalized to match the live time. A $\gamma$-ray from the decay of ${ }^{239} \mathrm{Pu}$ is visible near $E_{\gamma}=52 \mathrm{keV}$.

\section{REFERENCES}

1. R.O. Nelson, J.A. Becker, D.E. Archer, L.A. Bernstein, G.D. Johns, W.S. Wilburn, W. Younes, D.M. Drake, R.S. Rundberg, S.A. Wender, K. Hauschild, S.W. Yates, and P.E. Garrett, "GEANIE at WNR/LANSCE-A New Instrument for Neutron Science," in International Conference on Nuclear Data for Science and Technology (May 19-24, 1997, Trieste, Italy), Los Alamos National Laboratory report LA-UR97-1824.

2. J.A. Becker and R.O. Nelson, "New Physics Opportunities with GEANIE at LANSCE/WNR," Nuclear Physics News 7 (1997), Los Alamos National Laboratory report LA-UR-97-1080. 
3. P. W. Lisowski, C. D. Bowman, G. J. Russell, and S. A. Wender "The Los Alamos National Laboratory Spallation Neutron Sources," Nuclear Science and Engineering 106, 208 (1990).

4. J. C. Lashley, M. S. Blau, K. P. Staudhammer, and R. A. Pereyra, "In Situ Purification Alloying and Casting Methodology for Metallic Plutonium," Journal of Nuclear Materials 274, 315 (1999).

5. L. J. Mullins and J. A. Leary, "Fused-Salt Electrorefining of Molten Plutonium and Its Alloys by the LAMEX Process," Industrial and Engineering Chemistry, Process Design and Development 4(4), 39 (1965).

6. L. A. Bernstein, J. A. Becker, P. E. Garrett, K. Hauschild, C. A. McGrath, D. P. McNabb, W. Younes, M. Devlin, N. Fotiades, G. D. Johns, R. O. Nelson, and W. S. Wilburn, "Measurement of Several ${ }^{239} \mathrm{Pu}(\mathrm{n}, \mathrm{xn})$ Partial Cross Sections for $\mathrm{x} \leq 3$ Using GEANIE at LANSCE/WNR," Lawrence Livermore National Laboratory report (in preparation). 
This report has been reproduced directly from the best available copy. It is available electronically on the Web (http://www.doe.gov/bridge).

Copies are available for sale to U.S. Department of Energy employees and contractors from-

Office of Scientific and Technical Information

P.O. Box 62

Oak Ridge, TN 37831

(423) 576-8401

Copies are available for sale to the public from-

National Technical Information Service

U.S. Department of Commerce

5285 Port Royal Road

Springfield, VA 22616

(800) 553-6847 


\section{Los Alamos \\ NAT IONAL LABORATORY \\ Los Alamos, New Mexico 87545}

